\title{
Modelling the Mechanical Behaviour of Municipal Solid Waste for an Engineered Landfill using Viscoelasticity and other Established Concepts
}

by

\author{
Robert Peter McDonald, EIT
}

\begin{abstract}
A thesis submitted to
the Faculty of Graduate and Postdoctoral Affairs

in partial fulfillment of the requirements for the degree of

Master of Applied Science
\end{abstract}

in

Civil Engineering

Department of Civil and Environmental Engineering Carleton University

Ottawa-Carleton Institute for Civil Engineering (OCICE)

April 2018

(C) 2018 Robert Peter McDonald 


\section{ABSTRACT}

The stabilization process in municipal solid waste (MSW) is inherently complex. In the short term, waste has an immediate elastic response due to surcharge loading. Long-term stabilization involves a secondary mechanical settlement phase and a thermally dependent biodegradation phase. It can be difficult to distinguish the time-dependent behaviour between these components.

Two composite compressibility models were developed to simulate settlement. One model was based on Marques et al. (2003) and another on viscoelasticity. Both models achieved correspondence with the field data for short-term and long-term settlement. Good consistency was achieved for the parameters across both optimized models. The parameters achieved for both model types are all within acceptable limits from literature for MSW. The Marques et al. (2003) based model allowed a more rigid transition between immediate elastic response and time-dependent settlement. The viscoelastic model proved more consistent in reproducing the transition patterns seen in real MSW displacement. 


\section{ACKNOWLEDGEMENTS}

I would like to thank my supervisors, Dr Paul Van Geel and Dr Shawn Kenny, for all their guidance, teaching, and insight on this research. Without their consistent support and patience I would not be where I am today. I would also like to thank Courtney Berquist, who had the task of spending long road trips to the Ste. Sophie landfill with me, and joining me on our academic journey through our Master's degree. I would also like to thank my parents and my brothers for their ongoing encouragement in my academic pursuits. Finally, I would like to thank all my graduate friends and colleagues who got me through my entire time spent at Carleton University, in particular Michael Elten, Isis Bennet, Natalie Miller, Melanie Fillingham, Gurparam Kang, Amanda Burri, and Brandon Leblanc. 


\section{Table of Contents}

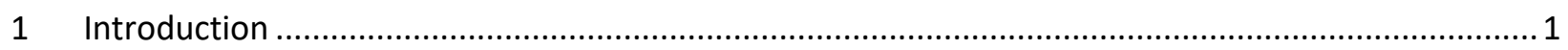

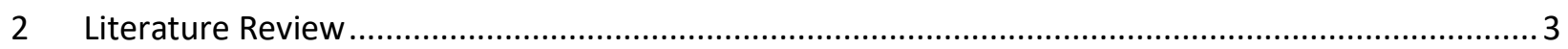

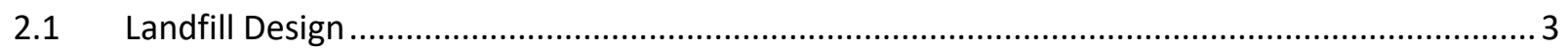

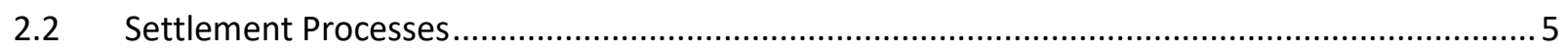

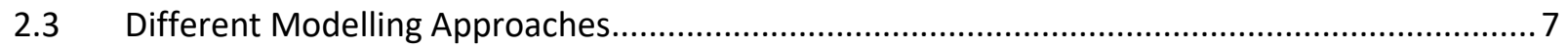

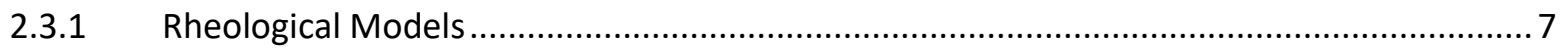

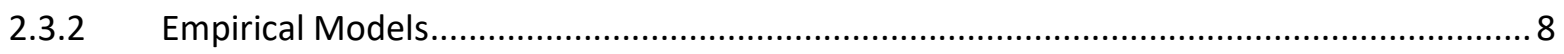

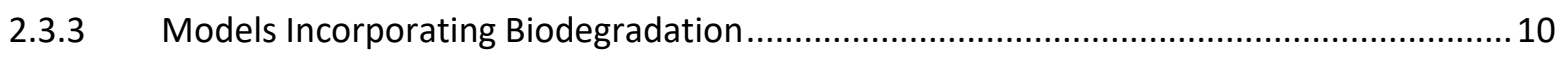

2.3.4 Soil Mechanics Based Models ................................................................................... 11

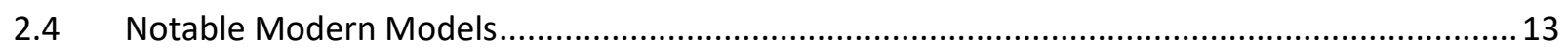

2.4.1 Machado Two Phase Constitutive Model (2002) ..........................................................16

2.4.2 Marques Composite Compressibility Model (Marques et al., 2003) ...............................16

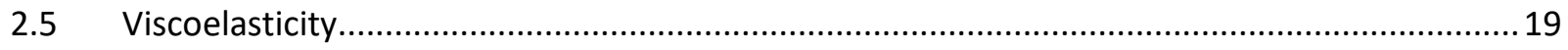

2.6 Predicting the Mechanical Behaviour of Solid Waste using Numerical Methods in Landfill

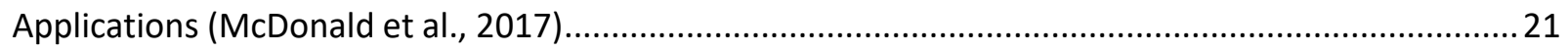

3 Ste. Sophie Landfill Site, Experimental Setup, and Field Data ......................................................23

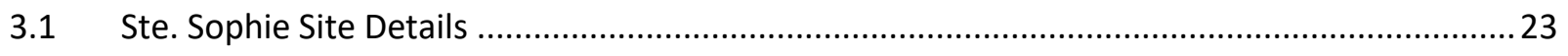

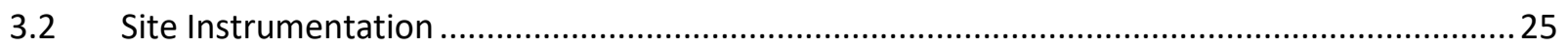

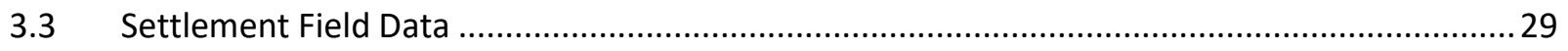

$4 \quad$ Finite Element Conceptual Models for Mechanical Settlement......................................................39

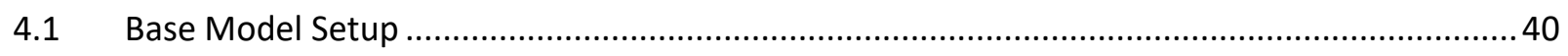

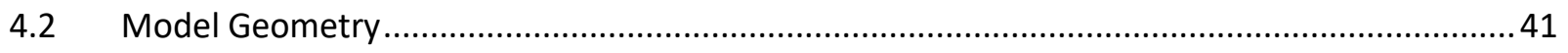

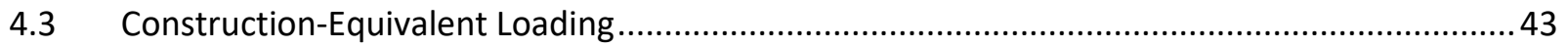

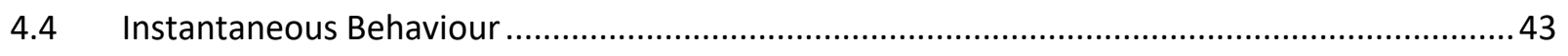

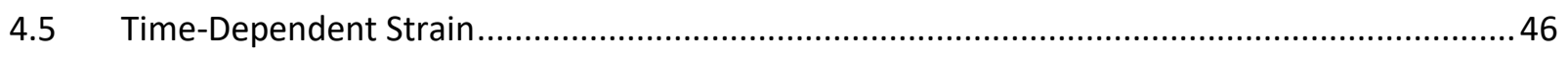

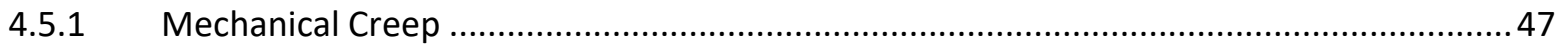

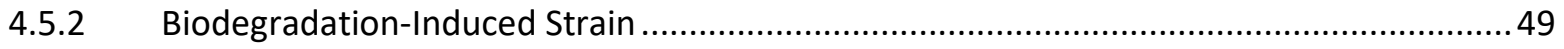

5 Mechanical Model Optimization, Results, and Discussion .........................................................52

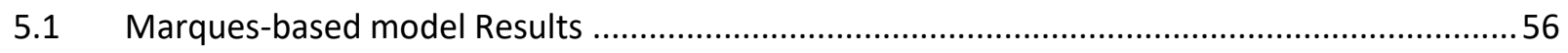

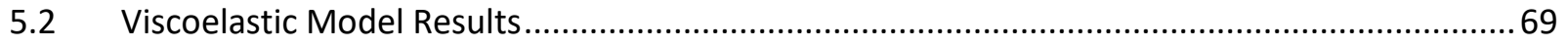

5.3 Comparison of Marques-based Model and Viscoelastic Model ........................................... 78 


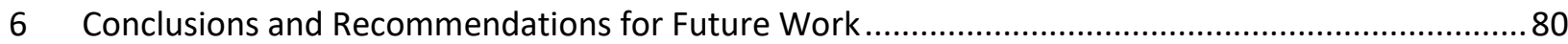

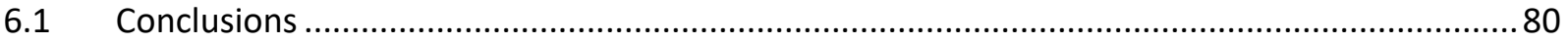

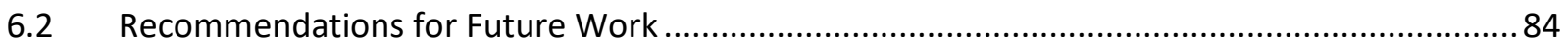

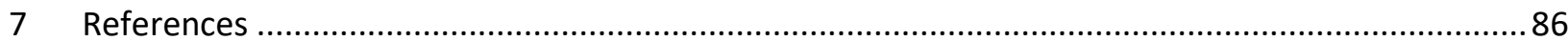

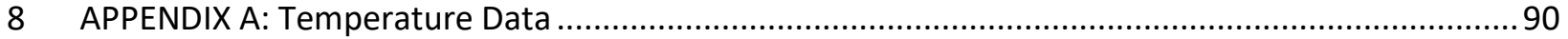

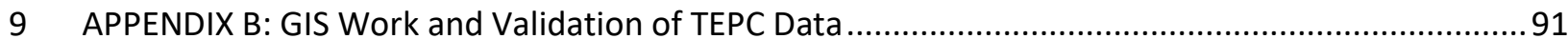




\section{List of Figures}

Figure 1 : Ste. Sophie Landfill Lift Placement. Red squares indicate instrument bundles. (Bonany, 2012). 5 Figure 2 : Three Parameter Solid, a combination of a spring in series with a Kelvin-Voight branch..........21

Figure 3: Kelvin-Voight branch, a combination of a spring and dashpot in parallel.................................21

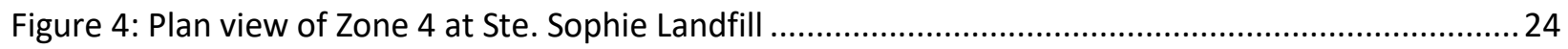

Figure 5: Instrument Bundle for Ste. Sophie Instrumentation .........................................................26

Figure 6: Ste. Sophie Full lift Cross Section with Bundle Locations (Berquist, 2017) ..............................22

Figure 7: Settlement Data by Elevation. Vertical bars indicate placement of waste lifts...........................30

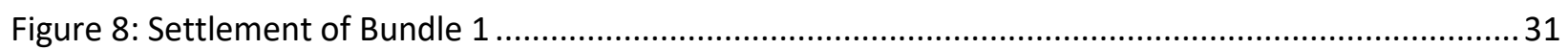

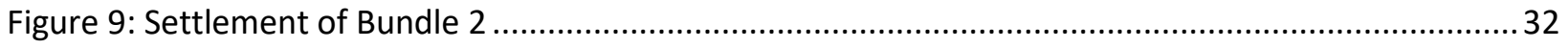

Figure 10: Settlement of Bundle 3. Green line indicates expected settlement rate without temperature effect. Orange line indicates start of new settlement rate due to delayed biodegradation......................33 Figure 11: Settlement of Bundle 4. Green line indicates expected settlement rate without temperature effect. Orange line indicates start of new settlement rate due to delayed biodegradation......................34 Figure 12: Settlement of Bundle 5. Green line indicates expected settlement rate without temperature effect. Orange line indicates start of new settlement rate due to delayed biodegradation......................34 Figure 13: Settlement of Bundle 6. Green line indicates expected settlement rate without temperature effect. Orange line indicates start of new settlement rate due to delayed biodegradation......................35

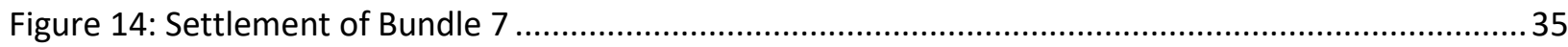

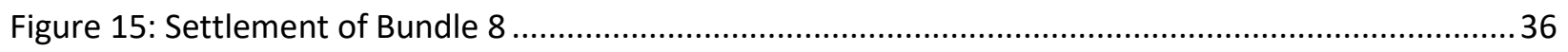

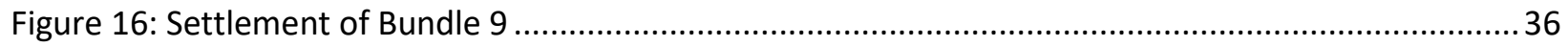

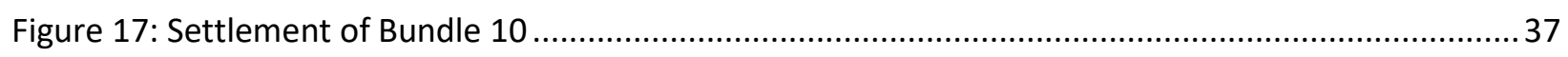

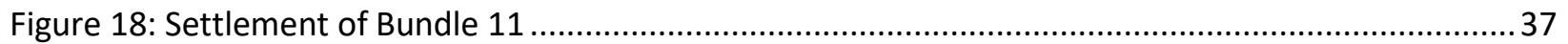

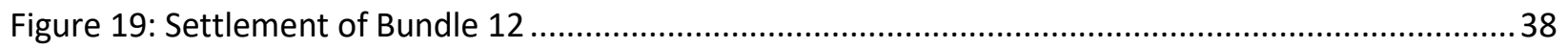

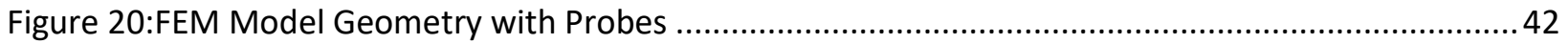

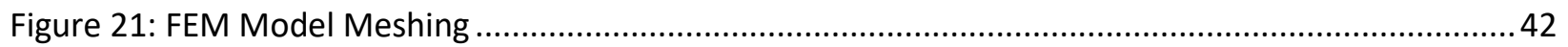

Figure 22: Cumulative Gamma Distribution Function for Expended Energy Curve Approximation ..........51

Figure 23: Cumulative Gamma Distribution Function for Expended Energy Curve Approximation ...........52

Figure 24: Marques Model results (MB\#) compared to field data before data probe location fix............54

Figure 25: Marques Model results (MB\#) compared to field data after data probe location fix ................55

Figure 26: Marques-based model optimized to B3, elevation of odd numbered bundles results.............57

Figure 27: Marques-based model optimized to B3, displacement of B3 results ....................................57

Figure 28: Marques-based model optimized to B4, elevation of even numbered bundles results ...........58

Figure 29: Marques-based model optimized to B4, displacement of B4 results......................................58

Figure 30: Marques-based model optimized to B3 and B4, elevation of odd numbered bundles results. 59

Figure 31: Marques-based model optimized to B3 and B4, displacement of B3 results.........................59

Figure 32: Marques-based model optimized to $B 3$ and B4, elevation of even numbered bundles results 60 Figure 33: Marques-based model optimized to B3 and B4, displacement of B4 results..........................60

Figure 34: Van Geel and Murray (2015)'s measured and simulated settlement for layers between Bundles 1-3 and Bundles 2-4 (BFM = Best Fit Model) ......................................................................65 
Figure 35: Van Geel and Murray (2015)'s prediction of layers between Bundles 6-8 and Bundles 5-7 using

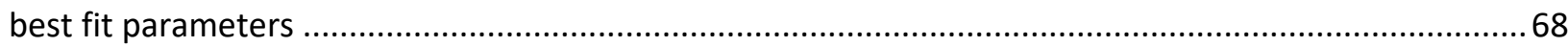

Figure 36: Viscoelastic model optimized to B3, elevation of odd numbered bundles results ..................69

Figure 37: Viscoelastic model optimized to B3, displacement of B3 results ......................................... 70

Figure 38: Viscoelastic model optimized to $B 4$, elevation of even numbered bundles results ..................70

Figure 39: Viscoelastic model optimized to B4, displacement of B4 results .........................................71

Figure 40: Viscoelastic model optimized to B3 and B4, elevation of odd numbered bundles results .......71

Figure 41: Viscoelastic model optimized to B3 and B4, displacement of B3 results .............................72

Figure 42: Viscoelastic model optimized to $B 3$ and B4, elevation of even numbered bundles results.......72

Figure 43: Viscoelastic model optimized to B3 and B4, displacement of B4 results ..............................73

Figure 44: Old viscoelastic model with Bundle 3 displacement results..................................................76

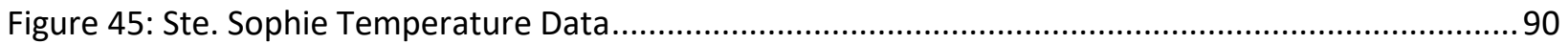

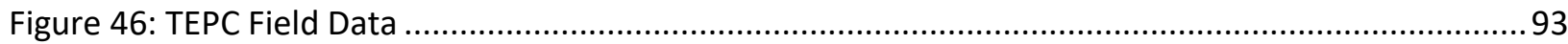

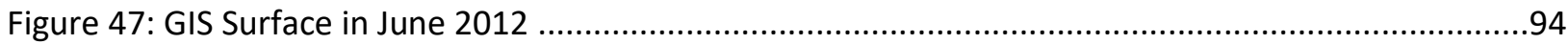

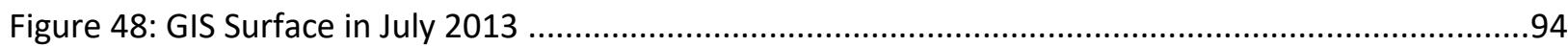

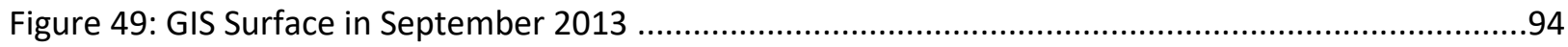

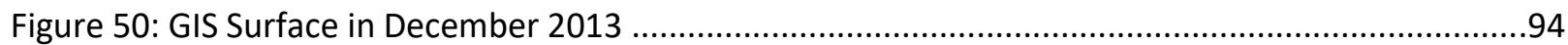

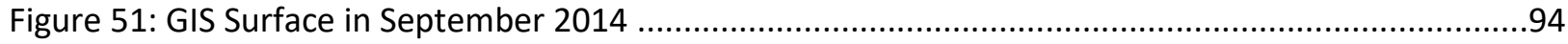

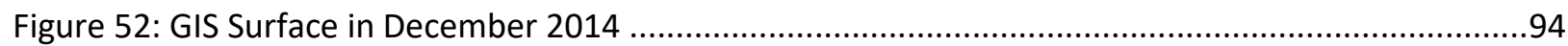

Figure 53: TEPC Field Data versus Boussinesq Solution Results .......................................................97

Figure 54: Stress profile from Viscoelastic Model for Ste. Sophie landfill .............................................98

Figure 55: September 2013 - December 2013 (Instrument bundles marked by star)..............................99

Figure 56: September 2014 - December 2014 (Instrument bundles marked by star).............................100

Figure 57: 3D Meshing of Ste. Sophie GIS Data in COMSOL Multiphysics............................................ 101 


\section{List of Tables}

Table 1: Surveyed locations of bundles and elevations during installation and total settlement as of September 2014 (Megalla, 2015) .

Table 2: Data Probe Adjustments per bundle, per optimization run ....................................................55

Table 3: Optimization Parameters for the Marques-based model ......................................................61

Table 4: B3 Optimization values with $N$ value constrained to expected trends for Marques-based model .63

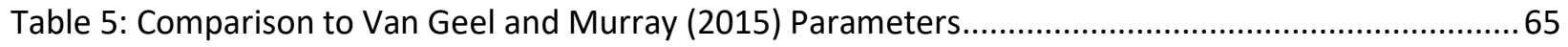

Table 6: Optimization Parameters for the viscoelastic model ................................................................ 73

Table 7: B3 Optimization values with $N$ value constrained to expected trends for viscoelastic model..... 74

Table 8: Average Parameters of Marques-based model and viscoelastic model optimizations ................79 


\section{Introduction}

Municipal Solid Waste (MSW) is a necessary side effect of a developed society, as is the responsibility of the proper disposal of said waste. MSW is defined as the wastes from single- and multifamily dwellings and include wastes generated from within the dwelling, from the yard, and from activities outside the dwelling (McBean et al., 1995). In practice, engineered landfills have proven to be an effective and environmentally conscious method for the disposal of MSW (McBean et al., 1995). Landfill sites can occupy areas ranging upwards of several hectares but are limited in construction by their potential for environmental impact. In Canada, landfills are regulated by provincial environmental agencies and environmental protection acts. These regulations impose design standards that require landfill operators to cap and close a landfill cell once it has reached a certain approved height (Canada Environmental Protection Act, 1990). In effect, it is increasingly important to maximize the storage capacity of existing landfills before the placement of their final covers so that landfill efficiency may be increased for environmental and economic benefit. If landfill operators can reclaim additional "airspace" within the approved landfill volume by promoting MSW settlement, a greater quantity of waste may be placed within a cell before installation of the final cover. The additional waste placed in turn leads to a more efficient and more profitable site for the site operators.

Operators can achieve additional settlement by promoting waste stabilization, i.e. by inducing intense mechanical compaction to reduce airspace, or by incorporating leachate recirculation to enhance biodegradation (McBean et al, 1995). Understanding the stabilization process is also important in the long-term operation of the landfill. The integrity of the final cover and the viability of the landfill site for redevelopment both rely on the magnitude and time-dependency of the MSW's site-specific stabilization. The stabilization process is highly variable due the number of factors of influence, including waste composition, duration of waste placement, season of placement, climate of landfill site, among 
other factors. This thesis aims to advance the understanding of the stabilization and settlement process amidst these factors.

A research project was initiated in 2009 by Dr. Paul Van Geel to study the waste stabilization processes occurring in landfills situated in northern climates. A landfill site in Ste. Sophie, Quebec, Canada, was selected and instrumented with 12 instrument bundles during the filling of a landfill cell between 2010 and 2012. The overall goal of this research project continues to be to develop a better understanding of the processes controlling waste stabilization in order to optimize landfill practices for increased waste stabilization. The various stabilization processes are being explored through the ongoing development of a multi-phase coupled thermal-hydraulic-biological-mechanical (THBM) computer model. The work by Bonany (2012), Megalla (2015), Doyle (2015), and Berquist (2017) have proposed a quasi-thermalmechanical-biological coupled landfill model, with a focus on the thermal and biological activity of the waste. This model can simulate change in biodegradation behaviour with the progression of temperatures within a waste profile over time, with the effects of frozen waste thaw, aerobic decomposition, and anaerobic decomposition considered; and offer an alternative to a simple timedependent approach for the biodegradation process independent of temperature. Other ThermalHydraulic-Biological-Mechanical (THBM) models in literature have also incorporated biodegradation components based on degradation fundamentals, such as McDougall (2008), Lobo et al. (2008), and White (2008).

In this thesis, two mechanical components for waste settlement are developed to link to the current thermal-biological model. The previous mechanical component coupled into the model by Doyle (2015) was an empirical settlement model to include the effect of settlement on the waste profile by prescribing mesh displacements. This allowed for a better comparison between simulated and field data. The new mechanical models proposed in this thesis aim to provide the groundwork for a more 
refined mechanical component. Major improvements from the previous mechanical model by Doyle (2015) include:

- The model can simulate the stabilization of all five waste lifts in a single simulation with model parameters optimized throughout the computations. Previously, when each waste lift was added, a new model was constructed, and a new simulation started using the previous results to define the initial conditions, which did not allow for the optimization of model parameters over the entire simulation period.

- The proposed model incorporates work by Berquist (2017) for a more site-specific, accurate, and less-generalized representation of long-term biological activity and an initial link between the biological-thermal and mechanical behaviour.

The mechanical models proposed herein will build on the mechanical models proposed in the literature by researchers such as Marques et al. (2003), Sowers (1973), and Edil et al. (1990). Other published models, such as Babu et al. $(2010,2011)$, feature similar settlement components or sources of influence, but provide a more complicated mechanical behaviour. The two mechanical models proposed in this thesis will strive for a more simplistic approach in order to maintain ease of adaptability to other landfill sites and coupling potential to the other stabilization components.

\section{Literature Review}

\subsection{Landfill Design}

In modern times, landfills exist as the final means of disposal for waste after recycling, composting, and other waste management diversion strategies are used. Historically, landfill practices began poorly with a "bury and forget" stigma that surrounded it well into the 20th century. Until the 1950's, municipal refuse disposal consisted of carless dumping operations without any engineering procedures or 
considerations to maximize usefulness of the disposal sites or provide environmental protection (McBean et al., 1995). As recognition of the environmental impact on groundwater, air quality, and greenhouse emissions grew, more legislation was put in to place to regulate landfill operations, which led to a more engineered approach to landfill design. The modern landfill incorporates geosynthetic liners, cover systems, gas collection systems, leachate collection/recirculation systems, and more to aid in the stabilization process and safe disposal of MSW (McBean et al., 1995).

Modern landfills employ the concept of controlled tipping, in which waste is placed into engineered cells covered with soil or other cover materials. This practice was implemented to change the physical characteristics of the buried solid wastes to make it less odorous, less accessible to vermin, and less dangerous to public health (McBean et al., 1995). All solid wastes received on site are spread and compacted in layers within a confined area. At the end of a working day (or more frequently if needed), this area is completely covered with a thin daily cover material. Soil is typically used for the daily cover, but some landfills use a temporary plastic cover that is rolled back the next working day. The compacted waste and cover material constitutes a cell. A series of adjoining cells, all of the same height, make up a lift (McBean et al., 1995). A lift is typically 3 - 5 m in height. A landfill will consist of several lifts; the Ste. Sophie landfill, which is discussed later in this thesis, consists of five lifts. Figure 1 shows the construction process of the Ste. Sophie landfill, which is typical of most landfills. 


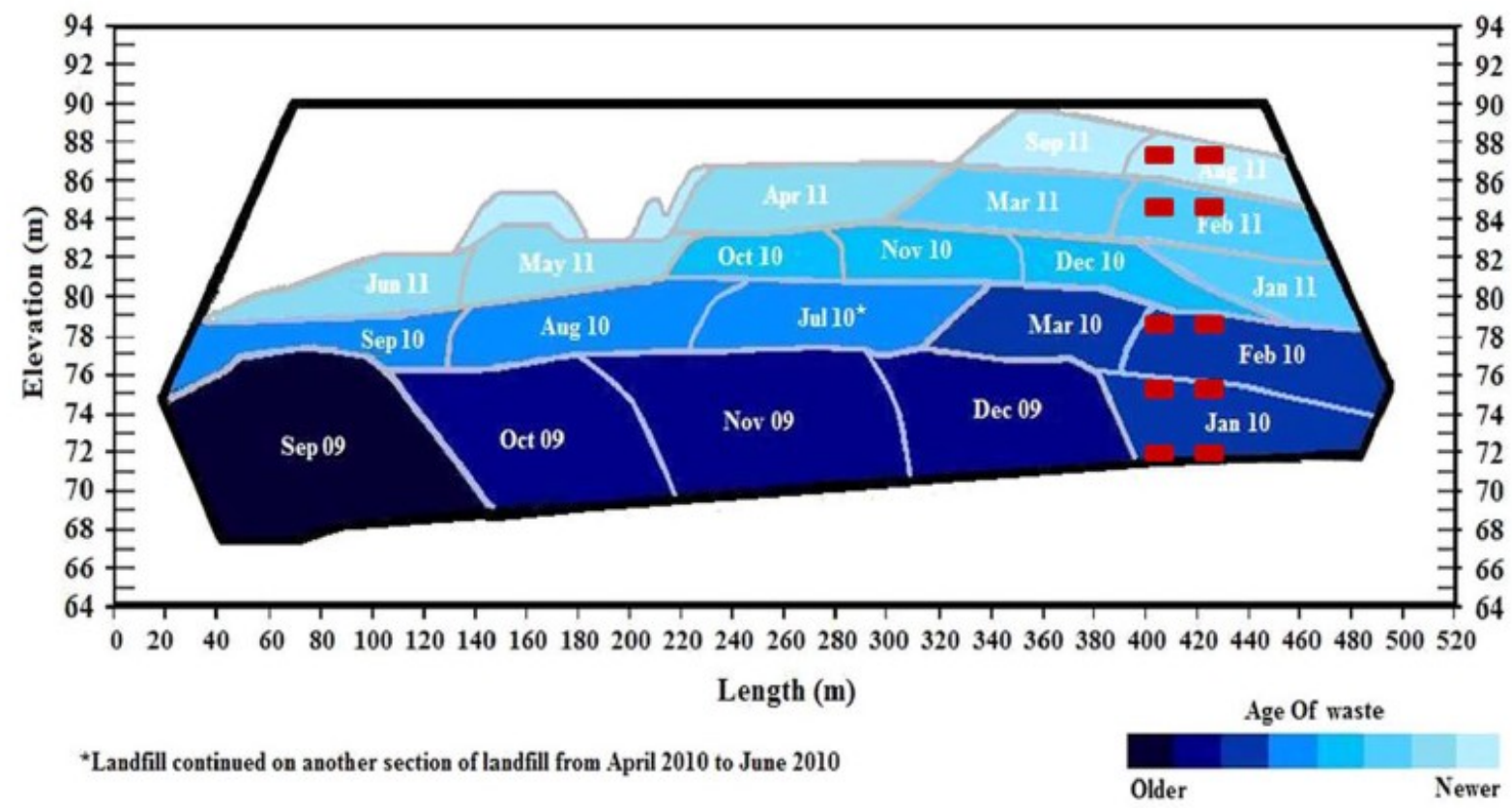

Figure 1 : Ste. Sophie Landfill Lift Placement. Red squares indicate instrument bundles. (Bonany, 2012).

\subsection{Settlement Processes}

Predicting and estimating MSW settlement is a critical component of landfill design and is widely studied. The mechanisms of settlement can be attributed to four main processes (Sowers, 1973; Sharma and Reddy, 2004):

1. Physical and Mechanical Processes: The reorientation and movement of particles and the filling and collapse of void spaces.

2. Chemical Processes: Corrosion, combustion, and oxidation within the material.

3. Dissolution Process: Dissolving soluble substances by percolating liquids and then forming leachate.

4. Biological Decomposition / Biochemical Decay: The organic parts decomposing with time according to controlling factors (temperature, humidity, and percentage of organics and nutrients within the waste). 
Although four distinct mechanisms of settlement are omnipresent within the waste, each mechanism does not contribute equally to the overall settlement. With the progress of waste literature over the years, it has been widely accepted that the mechanical processes and biological decomposition contribute the most to settlement, both in the short term and long-term results. As a result, many models have been reworked to better analyze the MSW based on stages of settlement, which include the processes that are most impactful. The processes analyzed now are the results of physical and biochemical mechanisms. These processes are generally referred to as : (1) immediate compression, (2) mechanical creep, and (3) biocompression (Sowers, 1973; El-Fadel and Khoury, 2000; Marques et al. 2003; Sivakumar Babu et al., 2010, Bareither et al., 2012). Immediate compression is the instantaneous settlement response to the applied loads, which is generally associated with the compaction of void space and accumulation of vertical strain. Consolidation and accumulated vertical strain continues to accumulate more slowly afterwards, due to mechanical creep and biocompression. Mechanical creep is a physical process where void space decreases due to particle slippage and yielding of the waste's materials. Biocompression is a biological process where the waste's organics undergo anaerobic or aerobic decomposition. The decomposition process notably requires a certain level of organic activity, which is temperature dependent, and will occur less at lower temperatures and not at all with frozen waste.

In relation to soil mechanics principles, biocompression and mechanical creep together represent behaviour analogous to the secondary compression seen in Terzaghi's consolidation theory (Terzaghi, K., 1943). It is this secondary compression that accounts for most of the landfill settlement over time, and is discussed later in Section 2.3.4. However, there is no correlation between immediate compression and Terzaghi's primary compression, despite the terms being interchangeably used across various MSW literature. With low permeability soils, primary compression occurs as the applied stress is initially handled by the pore water pressure, which gradually dissipates and transfers the stress to the soil 
skeleton; however modern landfills are usually designed to remain largely unsaturated - so this would not occur (Bareither et al., 2010). Additionally, studies show that the hydraulic conductivity of waste is around that of gravel and sand (El-Fadel and Khoury, 2000); meaning that excess pore water pressure would dissipate rapidly when loading occurs - further affirming that 'primary compression' or 'primary consolidation' as defined in Terzaghi's model does not effectively contribute to the settlement. However, due to the variability of MSW, not all landfills fit this criterion, and full consolidation models for MSW are still present and used in literature. These consolidation models have had some success in replicating settlement patterns of MSW, although the theory behind the models may not always be adequately applied to waste.

\subsection{Different Modelling Approaches}

To account for the settlement processes discussed in the previous Section, many models have been developed and published to account for one or more of the processes. Most models can be classified into four broad categories: (1) rheological models; (2) empirical models; (3) models incorporating biodegradation; and (4) soil-mechanics based models. Many models fall into multiple categories, as they superimpose or combine approaches; for example, an empirical component for instantaneous compression and a combined biodegradation/soils-based component for secondary compression.

\subsubsection{Rheological Models}

Rheological models use a phenomenological approach to duplicate the mechanical behaviour of materials. Typically, a system of theoretical mechanical models is created to mathematically describe the settlement behaviour of the material. These mechanical models are composed of elements such as sliders, springs, or dashpots - either in series or parallel, and can exhibit linear or non-linear behaviour. The modelling approach assumes the material to be continuous and homogeneous, which does not completely replicate the reality of MSW. Another drawback in using rheological models to simulate the 
mechanical behaviour of MSW, is the derivation of spring and dashpot parameters, which are stressdependent . However, rheological models can account for the effects of large early-stage consolidation and are also effective at separating the elastic and plastic settlement components.

The most widely referenced model in literature is the Gibson and Lo (1961) model. This model was particularly useful in predicting the settlement of peats (Gibson and Lo, 1961), which possess similar compressibility characteristics to MSW, as show by Edil et al. (1990). The model contains a spring to model the immediate compression, followed by a spring and dashpot in parallel to model the secondary compression over time. The behaviour of the model is expressed in Equation 2-A.

Equation 2-A

$$
S(t)=H_{0} \Delta \sigma\left[a+b\left(1-e^{-(\lambda / b) t}\right)\right]
$$

where $\mathrm{S}(\mathrm{t})=$ settlement, $\mathrm{m}$;

$$
\begin{aligned}
& \mathrm{H}_{0}=\text { initial height of refuse, } \mathrm{m} ; \\
& \Delta \sigma=\text { compressive stress, } \mathrm{kPa} ; \\
& \mathrm{a}=\text { primary compressibility parameter, } 1 / \mathrm{kPa} ; \\
& \mathrm{b}=\text { secondary compressibility parameter, } 1 / \mathrm{kPa} ; \\
& \lambda / \mathrm{b}=\text { rate of secondary compression, } 1 / \mathrm{day} ; \\
& \mathrm{t}=\text { time since load application, day }
\end{aligned}
$$

\subsubsection{Empirical Models}

Arising from uncertainty in using theoretical formulations to predict MSW settlement, empirical models have been developed to simulate general waste settlement behaviour. Unfortunately these empirical 
parameters are typically very site-specific and generally do not have any physical significance. Matching mathematical equations to MSW behaviour has somewhat limited success unless additional behavioural data are incorporated, such as distinguishing or parsing settlement phases or modes. The two most popular empirical models referenced throughout literature are the power creep model (Edil et al., 1990), and the hyperbolic function model (Ling et al., 1998). The power creep model is a simple relation for time dependant deformation under constant stress, shown in Equation 2-B. The hyperbolic function model computes settlement at a given time if the ultimate settlement of the landfill is previously known and thus is not a predictive tool. In comparison to other proposed empirical functions (e.g. logarithmic function and power function), Ling et al (1998) found better prediction of long-term settlement with the hyperbolic function. The hyperbolic function model is expressed in Equation 2-C:

Equation 2-B

$$
S(t)=H_{0} \Delta \sigma M\left(t / t_{r}\right)^{N}
$$

Equation 2-C

$$
S(t)=\frac{t}{1 / \rho_{0}+t / S_{u l t}}
$$

where $t=$ time since beginning of filling, day;

$\mathrm{M}=$ reference compressibility, $\mathrm{kPa}-1$;

$\mathrm{N}=$ rate of compression;

$t_{r}=$ reference time, day (usually 1 );

$\rho_{0}=$ initial rate of settlement, $\mathrm{m} / \mathrm{day}$;

$\mathrm{S}_{\mathrm{ult}}=$ ultimate settlement, $\mathrm{m}$; 


\subsubsection{Models Incorporating Biodegradation}

When analyzing for long-term settlement, the secondary compression component is usually the major contributor to the total settlement. Within secondary compression, two time-dependent processes are notable : the biodegradation-induced settlement and the mechanical creep. Often, the biodegradation fraction is considered the largest contributor towards total settlement, depending on organic content of the waste. In some models, such as Machado et al. (2008), the effect of biodegradation on the mechanical response of MSW is considered, but the effect of mechanical creep is omitted - assuming the biodegradation component dominates the secondary compression. Separation of these two timedependent behaviours from field data settlement is difficult, unless more refined field data is acquired. Generally, to model the biodegradation contribution, Park and Lee (1997) made a basic assumption : the settlement rate is assumed to be the amount of subsidence that is directly proportional to the amount of solids solubilized. Because the solubilization of organic solids is often expressed using first order kinetics, the biodegradation-induced settlement is expressed mathematically using first order kinetics as well. Park and Lee's settlement model is expressed in Equation 2-D and Equation 2-E. The summation of strains from both equations yield the total compressive strain, and can be used with the initial height of the waste to determine the settlement. However, this method can become challenging, as determining the kinetic coefficients - and their variation with environmental conditions - is quite difficult in landfills.

Equation 2-A

$$
\varepsilon(t)_{m e c h}=C_{\alpha} \log \left(\frac{t+\Delta t}{t}\right)
$$

Equation 2-B

$$
\varepsilon(t)_{d e c}=\varepsilon_{t o t_{-} d e c}\left(1-e^{k 1}\right)
$$

where $\varepsilon(t)_{\text {mech }}$ is the mechanical compression

$\varepsilon(t)_{d e c}$ is the decomposition compression 
$\varepsilon_{\text {tot_dec }}$ is total amount of decomposition that will occur due to decomposition of the biological waste (=7.2 to $6.1 \%)$

$C_{\alpha}$ is the rate of secondary compression

$\mathrm{k}_{1}=$ first order composition strain rate constant/time $(=2.37$ to $1.35 /$ year $)$

\subsubsection{Soil Mechanics Based Models}

Many models prefer the conventional approach and attempt to match the physical behaviour of MSW with theories derived in geotechnique. As mentioned previously, MSW settlement is often separated mathematically into primary and secondary compression processes, which comes from Terzaghi's theory of consolidation in the field of soil mechanics. However for MSW, the deformation is a mechanism of compaction, rather than of consolidation. In the study of landfill settlement, Sowers (1968) identifies the waste being compressed by its self weight, overburden, and external loads (such as that induced by compaction), leading to the reduction of void space. Sowers would then be the first to apply a soil mechanics based model to estimate the settlement of MSW, by using Terzaghi's consolidation theory and extending it, to relate settlement with effective stress, with a compression index (Sowers, 1973). The general form of the model is illustrated in Equation 2-F; the second term of the equation is dubbed 'the extension of Terzaghi's theory'.

Equation 2-C

$$
S(t)=\frac{H_{i} C_{c}}{1+e_{0}} \log \frac{P_{0}+\Delta P}{P_{0}}+\frac{H_{p} C_{p}}{1+e_{p}} \log \frac{t}{t_{p}}
$$

where $S(t)=$ settlement due to primary and secondary consolidation, $\mathrm{m}$;

$H_{i}=$ thickness of waste layer under consideration after initial compression, $m$;

$\mathrm{C}_{\mathrm{c}}=$ primary compression index; slope of e-log p curve; 


$$
\begin{aligned}
& e_{0}=\text { void ratio after initial compression, } \mathrm{m}^{3} / \mathrm{m}^{3} ; \\
& P_{0}=\text { existing overburden pressure at mid level of layer, } \mathrm{kPa} ; \\
& \Delta P=\text { increment of overburden pressure at midlevel of layer, } \mathrm{kN} / \mathrm{m} 2 \\
& H_{p}=\text { thickness of refuse after primary consolidation, } \mathrm{m} ; \\
& C_{a}=\text { secondary compression index; slope of e-log t curve; } \\
& e_{p}=\text { void ratio after primary consolidation, } \mathrm{m}^{3} / \mathrm{m}^{3} ; \\
& t=\text { time, } t>t_{p}, \text { days; } \\
& t_{p}=\text { time for primary consolidation to occur, days (usually } 30 \text { ). }
\end{aligned}
$$

Because of its simplicity, practical application, and the familiarity of the consolidation approach, the Sowers model is widely used by practicing engineers. Although its simplicity in form is due to many assumptions made in the classic Terzaghi theory, which lose validity when applied to MSW, which are discussed in the following paragraph.

Terzaghi's theory assumes soil is homogenous and saturated, whereas MSW is heterogeneous and typically unsaturated. Soil particles and pore water are assumed to be incompressible while MSW can contain components that are highly compressible. Terzaghi's settlement is assumed to be onedimensional, whereas the settlement of MSW is three-, or at best two-, dimensional due to the heterogeneity of waste and the non-uniform loading/placement procedure. It is assumed only small strain and velocities are involved; this is not true for MSW that can be very compressible. In Terzaghi's assumptions, the relationship between void ratio and effective stress is assumed to be linear, but in application to MSW, many studies have shown the compressibility-time relationship of MSW varies heavily with the load increment ratio, showing non-linear compaction behaviour (El-Fadel and Khoury, 
2000); and additionally, the compressibility of MSW skeleton fails the analogous comparison of a linear spring in many cases. Finally, Terzaghi's theory assumes a constant coefficient of consolidation at small strain for a particular load increment; however, due to secondary compression, the void ratio - at a constant stress - will continue to decrease even after the quick dissipation of pore pressure (El-Fadel and Khoury, 2000).

Many of these assumptions fail due to a few common landfill properties: landfills are not typically saturated, MSW particles and voids are much larger than those of soil, material properties are in flux due to chemical processes, materials are highly compressible, and a significant amount of their settlement is attributed to biodegradation and creep. The highly compressible skeleton of MSW means primary and secondary compression occur simultaneously, making it difficult to identify the contributions from each in practice. Additionally, implementing Sowers method requires many precise parameters of the waste, which heterogeneity and fluxing material properties make difficult to obtain.

\subsection{Notable Modern Models}

Sower's model was based on measurements up to only 15 months, but the majority of models developed for MSW since, owe inspiration or credit to Sowers (1973) method. Most early models began as an improvement to Sower's, to fill in or replace inaccurate gaps left in the initial model. Other models created new approaches to better replicate the complexity of waste behaviour. With a complex material such as waste - with an equally complex combination of settlement mechanisms - the need for more complex models arise. While a simple model can have difficulty replicating the variability and complexity of settlement behaviour, an overly complex model can become too site-specific and lack applicability to a wider range of landfills.

Many distinct approaches have been proposed across literature to effectively model the stabilization process of MSW. The LDAT model from the University of Southampton (e.g. White and Beaven, 2013; 
White et al., 2014) is an example of a comprehensive, modular model that links the previously mentioned settlement mechanisms as well as other chemical and biological processes. The model computes settlement via a simple bulk modulus relationship in which the modulus is linked to dry density which varies over time due to biodegradation (Hudson et al., 2004). Other models, such as Liu et al. (2006) and Hettiarachchi et al. (2007) estimated settlement from the reduction of solids due to biodegradation and to the gas pressures generated from biodegradation. Marques at al. (2003) proposed a settlement model which includes three distinct and explicit expressions for the three previously noted processes of settlement; immediate compression, mechanical creep, and biocompression. Gourc et al. (2010) proposed a model with time-dependent mechanical settlement modeled as mechanical creep and the time-dependent biodegradation behaviour was linked to two different gas generations models, which were dependent on waste characteristics.

Any of the aforementioned models are impressive in their efforts to match data recorded from one particular landfill, but an ideal model is applicable across a wide array of landfills, given sufficient fitting parameters or waste characteristics. Simões and Catapreta (2013) used three models to simulate six years of settlement data from the top of a $3.2 \mathrm{~m}$ waste profile in Brazil. They compared a rheological model by Edil et al. (1990), a hyperbolic function by Ling et al. (1998), and the composite compressibility model proposed by Marques et al. (2003). They fit the models to the settlement data up to 1, 3, and 6 years and showed that the best fit parameters experienced significant variance depending on the timeframe used. The best fit parameters from only the first year of data performed very poorly at predicting the 6 year settlement for all three models, with each model grossly underestimating the final settlement.

Bareither and Kwak (2015) similarly compared several previously proposed models to new settlement datasets. They used Gibson and Lo (1961), Marques et al. (2003), Hossain and Gabr (2005), Bjarngard 
and Edgers (1990), Machado et al. (2008), Babu Sivakumar et al. (2010), Chen et al. (2010), Gourc et al. (2010) and Chen et al. (2012). They compared each of these models to settlement data captured at two field scale sites: Yolo (Yazdani et al., 2006) and Deer Track Bioreactor Experiment (Bareither et al., 2012). The Yolo dataset recorded surface elevation starting at 7 months after the placement of the final cover. The Yolo site dataset included results from both a control cell and a cell with a leachate recirculation system to enhance waste stabilization. Bareither and Kwak (2015) found that the models proposed by Gourc et al. (2010) and Marques et a. (2003) provided the best fit in terms of the coefficient of determination. The Deer Track Bioreactor Experiment was conducted in a 2.4 diameter lysimeter (height $8.2 \mathrm{~m}$ ) and measured settlement at four different plates of varying depths. The models were fit to record the waste settlement at each waste layer (i.e. the column of waste between each point of measurement), and to the overall settlement measured at the top of the waste profile. All models provided a good fit when parameters were fit for each layer, however, some models featured fitting parameters that varied from lift to lift. Bareither and Kwak (2015) determined that the model proposed by Gourc et al. (2010) provided the best fit, due to its lower number of optimized parameters, high statistical fit for the overall settlement data from the top of the waste profile, and because its model parameters were more physically based. Van Geel and Murray (2015) developed a three-component model based on Marques et al. (2003) using regressed parameters from the dataset of a lowermost waste lift placed at the Ste. Sophie Landfill in Quebec, Canada. Van Geel and Murray (2015) compared their model parameters to the values published by both Marques et al. (2003), for the Bandeirantes Landfill in Sao Paul, Brazil; and Barethier and Kwak (2015), for the Yolo site and Deer Track Bioreactor Experiment. Van Geel and Murray (2015) determined that both the regressed parameters by Marques et al. (2003) and from their own study provided a better estimate of settlement for typical field scenarios. 


\subsubsection{Machado Two Phase Constitutive Model (2002)}

In most models, the physical properties and mechanisms are handled by the concepts developed in soil mechanics and adapted for MSW. Achieving parameters for these methods proved difficult due to the heterogeneity of the waste and the biochemical changes taking place over time. Machado proposed a new model to represent the mechanical behaviour of MSW based off the theory of elasto-plasticity. Machado et al. (2002) reviewed the published behaviour of MSW in literature, and performed their own tri-axial compression tests for verification. The proposed model was centered around the principle of dividing the MSW into a coupled two-phase material, with each phase having its own particular constitutive model. The two phases proposed were: (1) the fibrous materials, composed of many types of polymers and plastic materials present in the waste; and (2) the MSW paste, composed of all other non-fibrous material - i.e. wood, organic compounds, rubber, glass, liquids, etc. The derivation and breakdown of this model is quite complex and will be omitted for brevity. The basic composition of waste is a parameter often easily obtained by landfill operators, and its use creates a more site-specific approach based on the specific attributes of the waste. This model however does not address the behaviour of secondary compression and instead adopts the generally accepted equations proposed by Ling et al. (1998). Another controversial omission of this model relates to the time effects observed in waste, such as the loss of shear strength over time. Machado et al. (2002)'s classification of wood as a 'non-fibrous material' is contrary to expectations, but the model appears to run under the assumption that the wood's organic fibers would decay into the MSW paste, whereas the plastic and polymer fibers would be more permanent. Regardless, the model is nonetheless impressive in introducing an overdue alternative approach to conceptualize the mechanical behaviour of the waste.

\subsubsection{Marques Composite Compressibility Model (Marques et al., 2003)}

Marques et al. (2003) noted the abundance of compression models preceding theirs, each of which addressed at least one of what they dubbed 'the three important mechanisms of MSW compression': (1) 
immediate response to applied loading, (2) time-dependent mechanical creep, and (3) biological decomposition of the waste. None of the proposed models prior to Marques' however, incorporates three separate expressions to explicitly account for all three important mechanisms. According to this model, the strain of the MSW can be expressed as the sum of the strain from all three mechanisms, as seen in Equation 2-G. The strain equations themselves (Equation 2-H) have been adopted from previous well-tested models, but modified for the one-dimensional settlement of a single layer or "lift". The performance of each lift would then be summed to provide the overall performance of all lifts in the landfill.

Equation 2-D

$$
\varepsilon=\varepsilon_{\mathrm{P}}+\varepsilon_{\mathrm{C}}+\varepsilon_{\mathrm{B}}
$$

where $\varepsilon=$ Strain of all three mechanisms

$\varepsilon_{\mathrm{P}}=$ Strain resulting from instantaneous applied load

$\varepsilon_{\mathrm{C}}=$ Time dependent strain due to mechanical creep

$\varepsilon_{\mathrm{B}}=$ Time dependent strain due to biodegradation

Each strain component can be expanded into a more useful form as seen in Equation 2-H. The instantaneous strain term expression is adopted from Sowers (1973), which has been used by York et al. (1977), Morris and Woods (1990), and several others. The time-dependent mechanical creep is adapted from the work of Gibson and Lo's (1961) model for secondary compression of clays, which was later applied to waste by Edil et al. (1990). The time dependent biological decomposition expression was proposed by Park and Lee (1997). 
Equation 2-E

$$
\varepsilon=C_{C}^{\prime} \log \frac{\sigma_{0}+\Delta \sigma}{\sigma_{0}}+b(\Delta \sigma)\left(1-e^{-c t^{\prime}}\right)+E_{D G}\left(1-e^{-d t^{\prime \prime}}\right)
$$

where $C_{C}^{\prime}=$ compression ratio;

$\sigma_{0}=$ initial vertical stress;

$\Delta \sigma=$ change in vertical stress;

$b=$ coefficient of mechanical creep;

$c=$ rate constant for mechanical creep;

$t^{\prime}=$ time since application of the stress increment, $\Delta$;

$E_{D G}=$ total amount of strain that can occur due to biological decomposition;

$d=$ rate constant for biological decomposition;

$t^{\prime \prime}=$ time since placement of waste in lift.

While this approach is not revolutionary in the conceptualization of MSW behaviour, the Marques model is notable due to its implementation. MSW is predominantly placed in landfills over periods of several years. In doing so, the waste is placed in a series of "lifts", which since they are placed and compacted at significantly different points in time, undergo unique settlement over time. The total settlement at the landfill surface at any time, $t$, can then be expressed as the sum of each of these lifts seen in Equation 2-I.

Equation 2-F

$$
S(t)=\sum_{i=1}^{N} \Delta H_{i}\left[\boldsymbol{\epsilon}_{\boldsymbol{P i}}+\boldsymbol{\epsilon}_{\boldsymbol{C} \boldsymbol{i}}(\boldsymbol{t})+\boldsymbol{\epsilon}_{\boldsymbol{B i}}(\boldsymbol{t})\right]
$$


where $S(t)=$ Settlement of the surface

$\mathrm{N}=$ Number of lifts in the landfill

$\Delta \mathrm{H}_{\mathrm{i}}=$ Initial thickness of compacted lift ' $i$ '

Separating the settlement into a series of lifts is ideal if studying MSW in landfill studies - especially if the study is ongoing during the placement of lifts, and not retroactive during post-closure of the landfill. Application of the model requires a time history of filling and a few other model parameters, noted above. It models the composite performance of waste and daily cover, and can easily be used to calculate the settlement of the surface of any lift in the landfill, or the settlements induced by any overlying surcharge pressures. Because Marques' approach is highly flexible and adaptable, it is a viable template for new models; Babu Sivakumar et al. $(2010,2011)$ modified the Marques et al. (2003) model by replacing the instantaneous compression term with a soil mechanics based elastic and plastic behaviour model.

\subsection{Viscoelasticity}

Many materials (under small strains) are described by Hooke's law of linear elasticity, where stress is proportional to strain by a relating modulus of elasticity. Materials that obey this trend are dubbed elastic materials. In contrast, in a viscous fluid, such as a honey, stress is proportional to the strain-rate by a relating viscosity. Viscoelastic materials exhibit both elastic and viscous properties; such properties include hysteresis (a pronounced influence on loading rate and history), creep (increasing strain under constant stress), and relaxation (decreasing stress under constant strain). Examples of viscoelastic materials include concrete, synthetic polymers, wood, and metals at elevated temperatures. Because MSW displays viscoelastic properties, notably creep and hysteresis, viscoelastic principles have been used to model the time-dependent settlement of MSW (Yu. et al., 2011). Viscoelastic principles have 
also been applied to frozen soils and ice across various literature (Li et al., 2011; Lackner et al., 2008), and should have similar applicability to frozen MSW.

Viscoelastic behaviour can be either linear or non-linear. The distinction between the two concerns the creep and relaxation behaviour; for a material to be considered linear, it is necessary, but not sufficient, for the measured creep or relaxation function to be independent of stress or strain (Lakes, 2009). Viscoelasticity is usually described using discrete elastic and viscous mechanical elements, such as springs and dashpots. The spring element will deform instantaneously by a certain amount under an applied force, whereas the dashpot element under the same force will deform more slowly, with faster behaviour being dictated by the magnitude of the stress and the viscosity of the dashpot. Viscoelastic behaviour can be demonstrated by combining these two types of elements. Mathematically, a spring is defined by its elastic stiffness, usually an elastic or shear modulus; whereas a dashpot is defined by its viscosity. A number of springs/dashpots can be added in series or parallel for a variety of possible patterns of viscoelastic behaviour. Two combinations of note in this thesis are the three-parameter solid (Figure 2) and the Kevlin-Voight body (Figure 3). The three-parameter solid contains a linear spring in series with a linear spring and dashpot in parallel. The component consisting of a spring and dashpot in parallel is called a Kelvin solid, or more commonly a Kevlin-Voight body or Kelvin-Voight branch. In the three-parameter solid, the Kevlin-Voight (KV) branch controls the time-dependent behaviour, while the initial spring in series controls the instantaneous response to load - i.e. the spring controls the impact response seen in most solid materials and the KV branch represents creep (Flügge, 1975). The three parameter solid is also remarkably similar to the rheological model proposed by Gibson and Lo (1961) and applied to waste by Edil et al. (1990). 


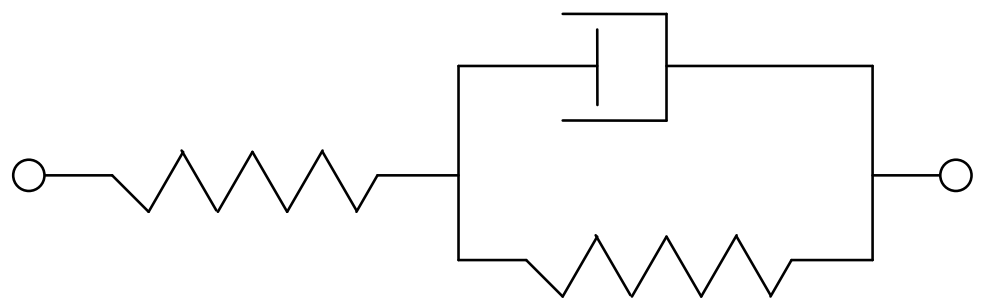

Figure 2 : Three Parameter Solid, a combination of a spring in series with a Kelvin-Voight branch

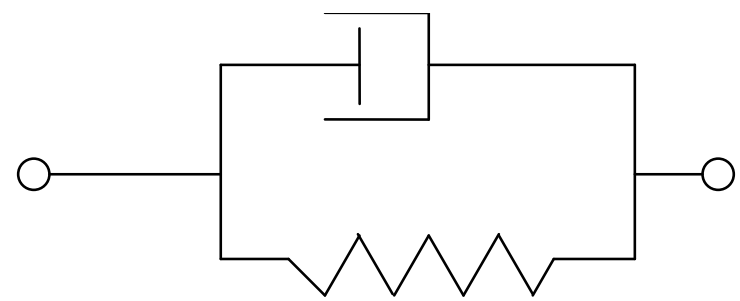

Figure 3: Kelvin-Voight branch, a combination of a spring and dashpot in parallel

\subsection{Predicting the Mechanical Behaviour of Solid Waste using Numerical}

Methods in Landfill Applications (McDonald et al., 2017)

The precursor to the work seen within this thesis was previously presented by the author in 2017 . This paper introduces early finite element versions of the Marques et al. (2003) based model and viscoelastic model presented later in this thesis.

McDonald et al. (2017) discusses the concept of complex versus simple modelling approaches for municipal solid waste. MSW, as a highly heterogeneous material with several simultaneous settlement modes, can be difficult to have represented by a numerical model that can be widely applied at different engineered landfill sites. However, it is often difficult to gauge the magnitude, separation, and significance of each settlement mode with simple models. McDonald et al. (2017) implements two numerical approaches into a finite element method (FEM) software; one model developed previously by Marques et al. (2003), and another more generalized model based on the concept of viscoelasticity. The viscoelastic model is idealized as a three parameter viscoelastic solid, which incorporates a linear spring 
and a Kelvin-Voight body. Both models were compared with their application to a landfill in Ste. Sophie, Quebec, Canada.

Both models performed adequately in matching the field data, but fared significantly better in matching the long-term trends over the short-term trends. The predictions for instantaneous response behaviour worsened over time with successive lifts applied. This behaviour could be improved in both models by potentially introducing a non-linear stress-strain relationship for the material, or including elastic material parameters which are reliant on changing factors of the waste over time. Possible factors of influence on the elastic material parameters include waste age, degree of decomposition, or degree of compaction, which were unaccounted for in the models. This is in agreement with observations by Beaven and Powrie (1995) in large-scale field experiments that exhibited variation in the elastic modulus with depth and age. A FEM material density that updates with biodegradation would also improve the model.

The most significant issue with the two models shown in McDonald et al. (2017), is the separation of the long-term settlement mechanisms; which is a common issue amongst many published MSW settlement models. Two time dependent mechanisms occur, mechanical creep and biodegradationinduced settlement. Trials of the models incorporated multiple KV branches in an attempt to separate the two long-term settlement mechanisms and reveal an apparent separation of two modes. However, without proper constraints to define and separate the components, the inclusion of additional KV branches worsened results. The biodegradation term should be linked to temperature with limits based on the organic content of the waste, among other factors. The data obtained from the Ste. Sophie landfill includes temperature data recorded at each bundle over time. This data could be used to fix the biodegradation behaviour separation with a temperature controlling the start and end of its timedependent effect. 


\section{Ste. Sophie Landfill Site, Experimental Setup, and Field Data}

\subsection{Ste. Sophie Site Details}

The research group of Dr. Paul Van Geel (which includes the author of this thesis) have been collecting data from Ste. Sophie Landfill since 2009. The landfill is located approximately $50 \mathrm{~km}$ northwest of Montreal, Quebec, and features a northern climate, typical to Canada, with temperatures as low as $30^{\circ} \mathrm{C}$ in the winter, and as high as $+30^{\circ} \mathrm{C}$ in the summer. As a result, the data recorded includes the effect of warm and frozen waste - which is a unique trait of this dataset compared to other published studies. The landfill is permitted to receive up to 1.0 million tonnes of waste each year, and takes in municipal solid waste, industrial waste, commercial/institutional waste, construction/demolition waste, automotive waste, and contaminated soils from nearby areas. The site incorporates a landfill gas collection system; approximately half the gas collected is sent to a nearby pulp and paper mill as an energy resource, while the other half is flared.

Van Geel's research group installed their instruments in the southern portion of Zone 4 at Ste. Sophie (Figure 4) before and during the filling stages of the zone. The first bundles were placed in November 2009, with additional bundles being installed between the placement of waste lifts; which lasted from January 2010 until mid 2012. The zone is filled primarily with municipal solid waste. This zone features horizontal gas collection but no leachate recirculation. The site features very good compaction, with an average waste density of $930 \mathrm{~kg} / \mathrm{m}^{3}$ achieved; good compaction is generally taken as $600 \mathrm{~kg} / \mathrm{m}^{3}$ (McBean et al., 1995). The landfill was designed to have a 30\% side slope and a $2 \%$ top slope. 


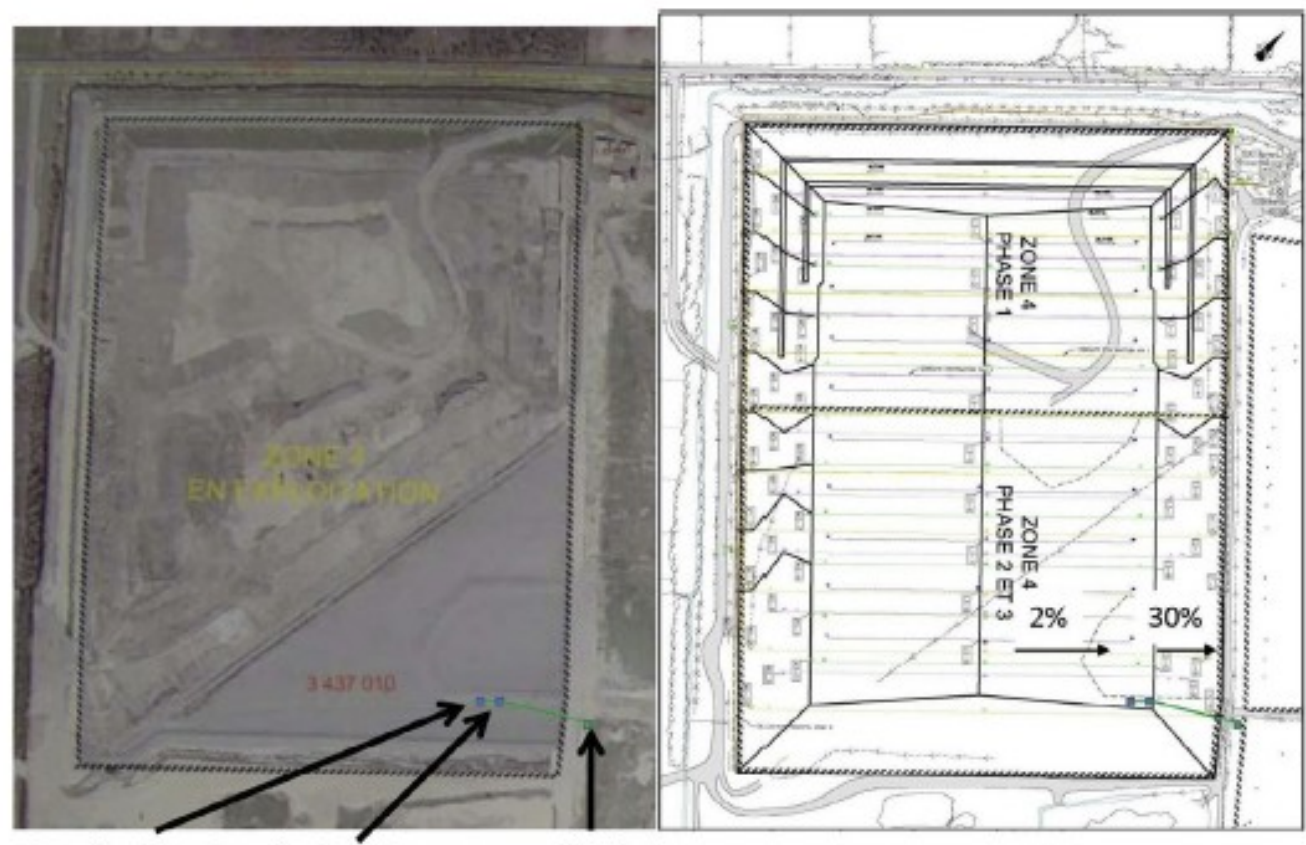

Bundle \#2 Bundle \# 1 Instrument Shed

Figure 4: Plan view of Zone 4 at Ste. Sophie Landfill

Figure 4 shows an aerial view of the zone, as well as the approximate location of the instrument shed and the two vertical columns where the field instruments were installed. The field instruments were planned to be installed just inside the top slope area in an effort to reduce the cable lengths and save on instrumentation costs. The cabling and tubing, which connect the field instruments to the instrument shed, accounted for $40 \%$ of the field equipment costs, and the installation of the field instruments in their present location allowed for a minimization of this cost. In regards to the mechanical behaviour, the instruments would have ideally been placed further into the middle of the landfill, or at least along an edge, instead of the corner where they were placed. The construction of the neighbouring landfill zone (Zone 5) and the effect of its proximity on the mechanical behaviour studied in this thesis is discussed in some detail in Appendix B. In this Appendix, it was determined by analyzing the quarterly GIS survey and TEPC dataset that only the waste (even if in a small or temporary pile) placed overlying the instrument bundles, or in close proximity, were producing significant stress readings (and therefore inducing mechanical settlement), and the larger waste lifts placed at a distance were far less significant. 


\subsection{Site Instrumentation}

As previously noted, two vertical columns of instruments bundles were installed during the filling stage of the landfill. Each column consists of 6 instrument bundles, for a total of 12 instrument bundles. At the time of installation, each instrument bundle was covered in $30-40 \mathrm{~cm}$ of sand for protection of the instruments. Each instrument bundle is comprised of a $60 \mathrm{~cm} \times 60 \mathrm{~cm}$ steel plate and outfitted with the following components:

- Total Earth Pressure Cell (TEPC) - to measure overburden earth pressure due to waste placed above the bundle. Also measures temperature.

- Oxygen Sensor - to measure the amount of oxygen present in the gas phase (\% by volume). Also measures temperature.

- Moisture and Electrical Conductivity Sensor - to measure moisture content of the sand covering the instrument bundle which should reflect the surrounding waste and leachate. Also measures temperature.

- Liquid Settlement Sensor - to measure the change in elevation of the bundle. Also measures temperature.

- Vibrating Wire Piezometer (Bundles 1, 2, 3, and 4 only) - to measure leachate mounding.

- Two Hollow Plastic Lines Connected to the instrument shed - to draw out leachate or landfill gas, or to inject air into the landfill.

A photograph of one of the instrument bundles installed is provided in Figure 5. 


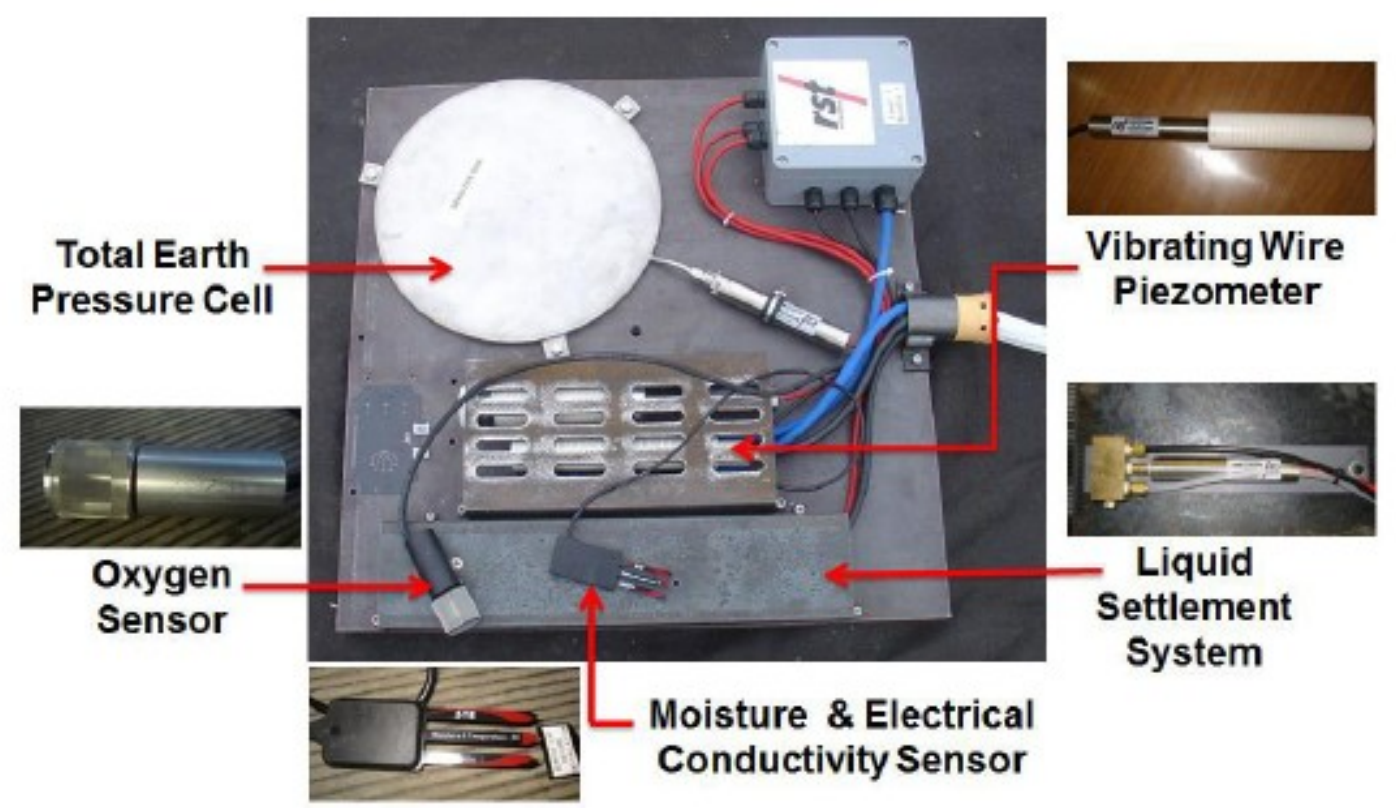

Figure 5: Instrument Bundle for Ste. Sophie Instrumentation

All of the sensors on the instrument bundle feed into a single junction box on the instrument bundle and connect to a single 24-pair cable (blue cable in Figure 5), which goes back to the instrument shed through a corrugated plastic pipe. The two plastic lines for gas and leachate testing also lead back to the instrument shed through corrugated pipe, but are also encased in a protective sheath inside the pipe. A similar protective sheath also covers the tubing used for the liquid settlement system, which also travels through the corrugated pipe back to the instrument shed. At the shed, the 24-pair cable feeds into a Campbell Scientific CR1000 FlexDAQ Datalogger. The data from the datalogger can be accessed on site by connecting a computer, or can be downloaded wirelessly offsite thanks to the inclusion of a modem connected to the datalogger.

The bundles were placed progressively in the waste profile as it was constructed in a series of lifts.

Figure 6 shows the bundle locations in the full waste lift profile of the landfill. It should be noted here however, that Figure 6 depicts the full height of each waste lift at placement, while in reality, each lift decreased in height over time before subsequent waste lifts were placed. 


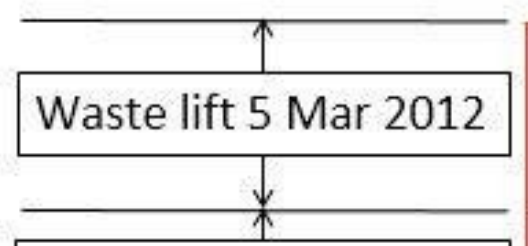

Waste lift 4 Aug 2011
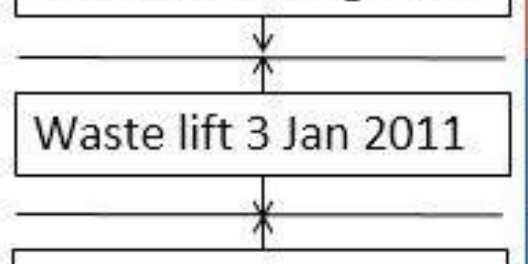

Waste lift 2 Dec 2010

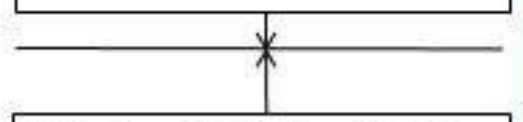

Waste lift 1 Jan 2010

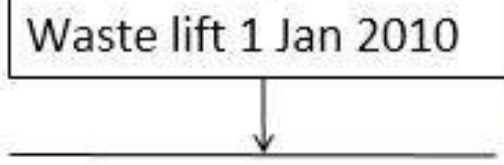

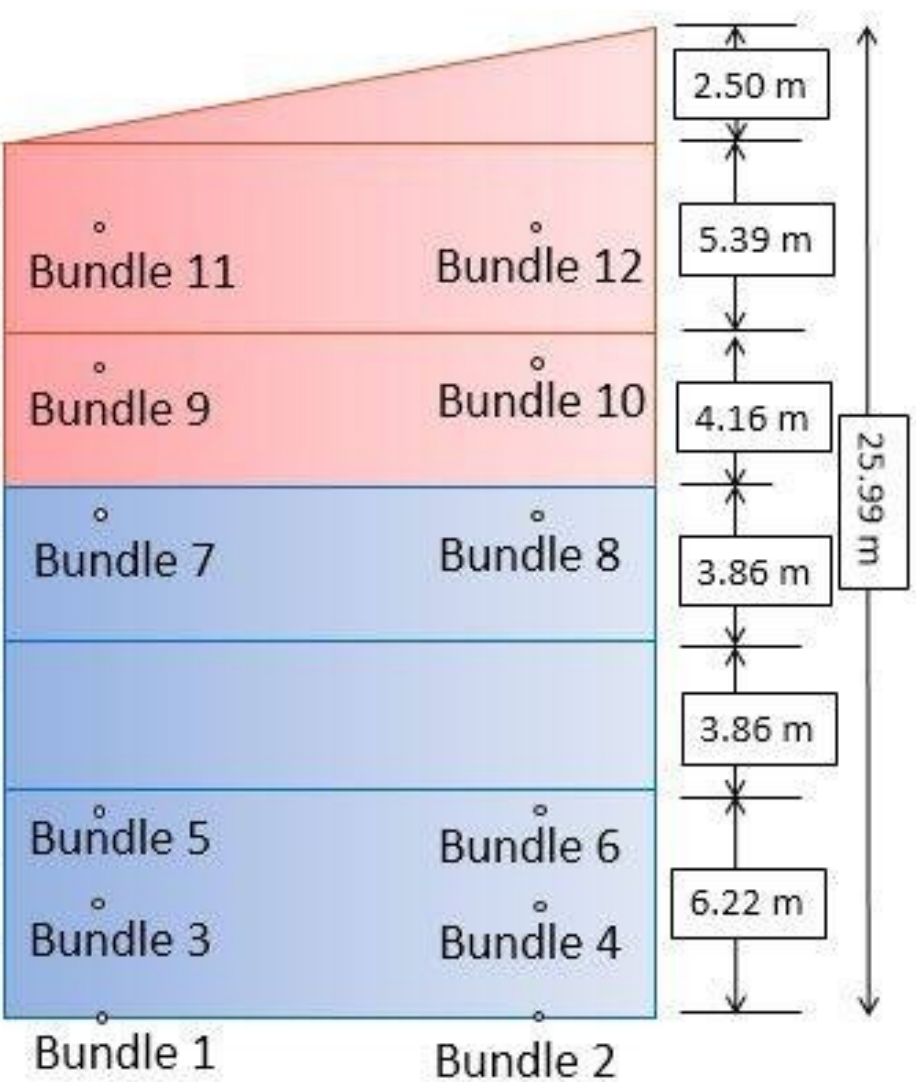

Figure 6: Ste. Sophie Full lift Cross Section with Bundle Locations (Berquist, 2017)

Bundles 1 and 2 were placed in November 2009, at a distance of 21.5m apart, at the base of the landfill, on top of the leachate collection system. Starting in January 2010, the first layer of waste placed onto the leachate collection system was approximately $1-1.5 \mathrm{~m}$ in thickness and was left uncompacted. Each following layer was $30-40 \mathrm{~cm}$ in thickness and compacted by $4-5$ passes of a sheepsfoot roller. Landfill operators placed half the first waste lift and permitted the installation of Bundles 3 and 4 at the centre of the waste lift in January 2010. Placement of the second half of the first waste lift continued immediately after the installation of Bundles 3 and 4 . Bundles 5 and 6 were placed approximately $0.5 \mathrm{~m}$ below the top of the first waste lift in June 2010. The second waste was placed in December 2010 and the third waste lift was placed shortly after in January 2011. Bundles 7 and 8 were installed approximately $0.5 \mathrm{~m}$ below the top of the third waste lift in February 2011. While the first three waste 
lifts were placed during the winter months and experienced freezing ambient conditions, the fourth waste lift was requested by the research team to be placed in the summer months under warmer conditions for a more diverse data set. The fourth waste lift was thus placed in August 2011 and Bundles 9 and 10 were installed in September 2011, approximately $1 \mathrm{~m}$ below the top of the lift. The fifth and final waste lift was placed starting in March 2012. Bundles 11 and 12 were placed in late May, and approximately $5 \mathrm{~m}$ of waste was added above the bundles to bring the top of the waste profile close to its permitted elevation. When Bundles 11 and 12 were placed, they were not placed in the same two vertical columns in which the rest of the bundles were placed; they were instead placed further towards the middle of the landfill. The reasons for offsetting Bundles 11 and 12 was to allow sufficient coverage under the side slope (the slope seen in Figure 6); and to move away from the end slope (which would be perpendicular to the slope in Figure 6) and a temporary road being used there. The road was eventually covered with waste. The specific location of each bundle can be seen in Table 1 below.

Table 1: Surveyed locations of bundles and elevations during installation and total settlement as of September 2014 (Megalla, 2015)

\begin{tabular}{|c|c|c|c|c|c|c|}
\hline Bundle & Northing & Easting & $\begin{array}{l}\text { Elevation - As } \\
\text { placed (masl) }\end{array}$ & $\begin{array}{c}\text { Distance from } \\
\text { landfill bottom as } \\
\text { placed (m) }\end{array}$ & $\begin{array}{c}\text { Elevation as of } \\
\mathbf{2 3 / 9 / 2 0 1 4}(\mathbf{m})\end{array}$ & $\begin{array}{c}\text { Total Settlement as } \\
\text { of 23/9/2014 (m) }\end{array}$ \\
\hline 1 & 5070938 & 273056.7 & 72.58 & 0 & 72.09 & 0.49 \\
\hline 2 & 5070925 & 273045.4 & 72.47 & 0 & 71.78 & 0.69 \\
\hline 3 & 5070938 & 273056.7 & 75.46 & 2.88 & 74.00 & 1.46 \\
\hline 4 & 5070925 & 273044.9 & 75.63 & 3.16 & 73.85 & 1.78 \\
\hline 5 & 5070939 & 273057.3 & 77.79 & 5.21 & 75.94 & 1.85 \\
\hline 6 & 5070925 & 273044.9 & 78.31 & 5.84 & 76.24 & 2.07 \\
\hline 7 & 5070938 & 273056.4 & 84.57 & 11.99 & 81.88 & 2.69 \\
\hline 8 & 5070925 & 273045.1 & 84.76 & 12.29 & 81.86 & 2.90 \\
\hline 9 & 5070938 & 273056.5 & 87.71 & 15.13 & 85.28 & 2.43 \\
\hline 10 & 5070925 & 273044.3 & 87.77 & 15.3 & 85.28 & 2.49 \\
\hline 11 & 5070940 & 273051.1 & 91.918 & 19.338 & 90.03 & 1.88 \\
\hline 12 & 5070930 & 273036.5 & 92.272 & 19.802 & 89.71 & 2.56 \\
\hline
\end{tabular}




\subsection{Settlement Field Data}

The majority of instruments remain in working condition even after almost 8 years of being entombed in waste. The moisture and electrical conductivity sensors failed within the first $6-12$ months and did not provide adequate data for analysis. The focus of this thesis was on replicating the settlement behaviour, and will be more thoroughly discussed in the following Section. Temperature data is included in Appendix $A$ as it pertains to the biodegradation-induced settlement behaviour, but was not a main focus of this thesis. Total earth pressure cell (TEPC) results would have been beneficial to the analysis, but are omitted due to the reliability of the data acquired. The TEPC data produced the expected trends under waste placement, however the magnitude of the values reported appear to overestimate the stress given the weight of overlying waste. Further discussion of TEPC results is included in Appendix B.

The settlement data is estimated using the liquid settlement system installed on each of the instrument bundles. The settlement sensor on each bundle consists of a pressure transducer that is hydraulically connected to a reference pressure recorded by a pressure transducer in the instrument shed. The instrument shed (as seen in Figure 4) is located outside the footprint of the landfill. As previously mentioned, the hydraulic connection between the transducers in the instrument bundles and the instrument shed for the liquid settlement system consists of tubing covered by a single protective sheath, which, along with the instrument cabling, is placed is a corrugated plastic pipe. This tubing is filled with a $50 \%$ water, $50 \%$ glycol solution to prevent freezing. The settlement data at any time is calculated by the pressure difference between the instrument bundle and the reference pressure in the instrument shed. The value of pressure difference at the time of placement is recorded and becomes the datum for zero displacement. The increase in differential pressure can then be converted to a settlement based on the density of the water/glycol. The pressure transducers are vibrating wire pressure transducers, corrected for temperature and have an accuracy of $<0.1 \%$ full scale, which is 170 $\mathrm{kPa}$ ( 17.3 m equivalent water head); $0.1 \%$ of full scale corresponds to approximately $1.7 \mathrm{~cm}$ of $50 \%$ 
water-glycol solution at $20^{\circ} \mathrm{C}$ (Van Geel and Murray, 2015). The settlement data recorded from November 2009 until March 2017 is presented in Figure 7.

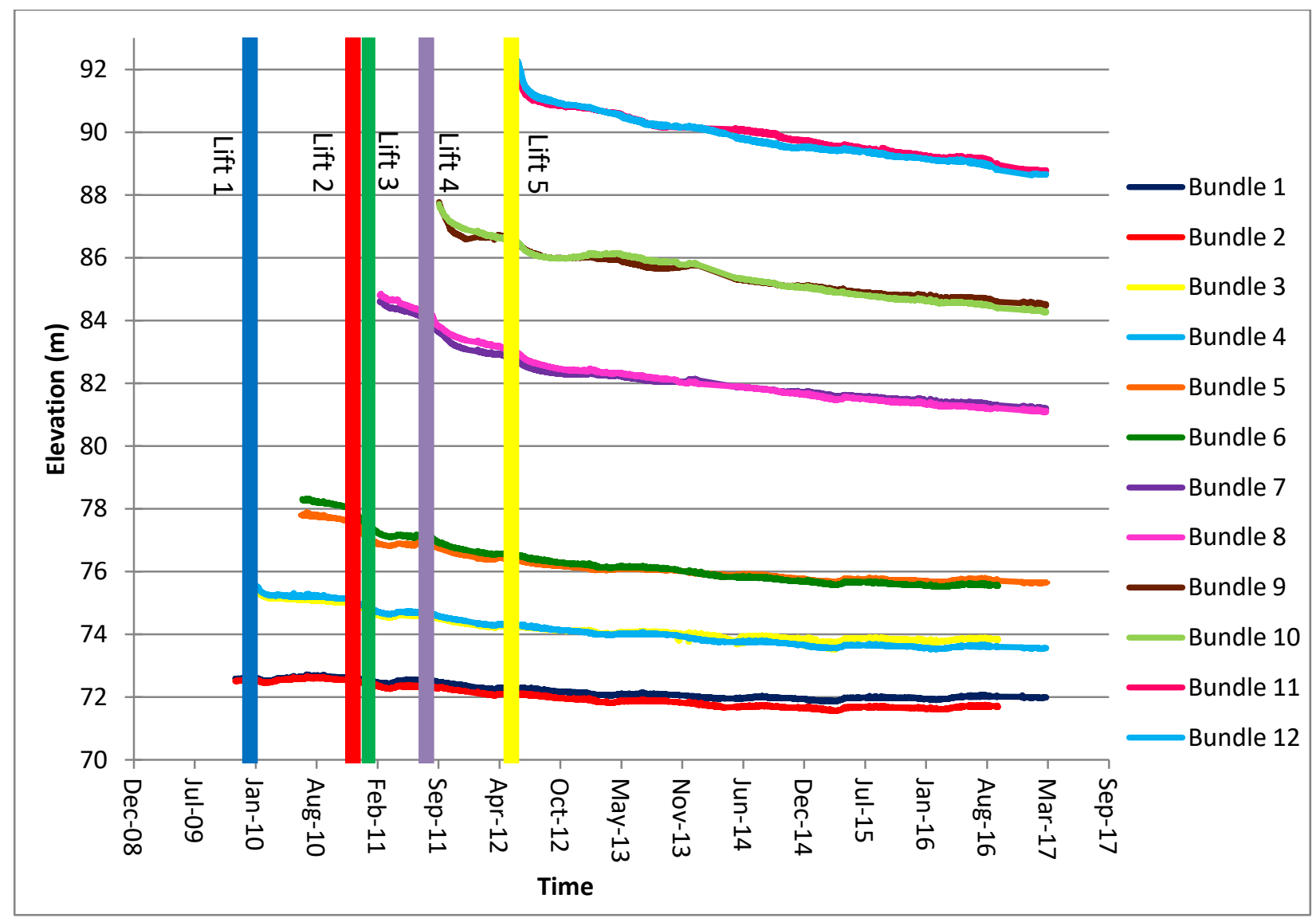

Figure 7: Settlement Data by Elevation. Vertical bars indicate placement of waste lifts.

Figure 7 shows the change in elevation of each instrument bundle due to settlement over time. The vertical bars depict the placement of each of the five waste lifts. Notably, at the placement of each waste lift, the instrument bundle locations undergo a sharp increase in settlement due to an instantaneous compression component, followed by a prolonged increase in settlement due to mechanical creep and biodegradation effects. These effects are more apparent when the settlement is depicted as cumulative settlement without including the elevation, which can be seen in the following plots. The lower most bundles (Bundles 1 and 2) were placed under the waste profile, and their settlement is representative of the consolidation of the clay unit which underlies the landfill. A design 
engineer for the landfill site predicted that this clay unit would yield a settlement of approximately $2 \mathrm{~m}$ due to consolidation effects under the increased vertical stress of the landfill. The settlement of these two lowermost bundles is shown in Figure 10 and 11.

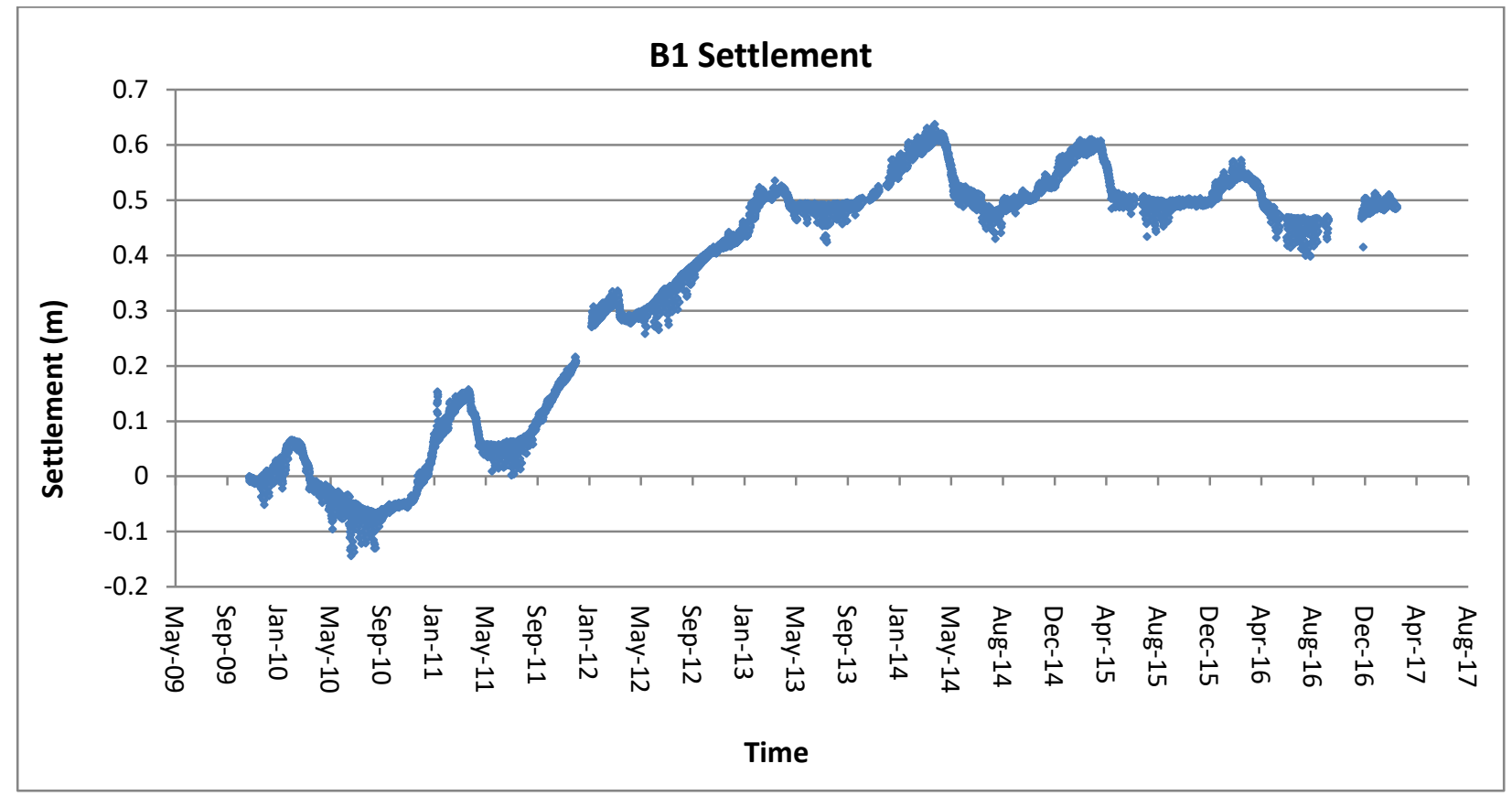

Figure 8: Settlement of Bundle 1 


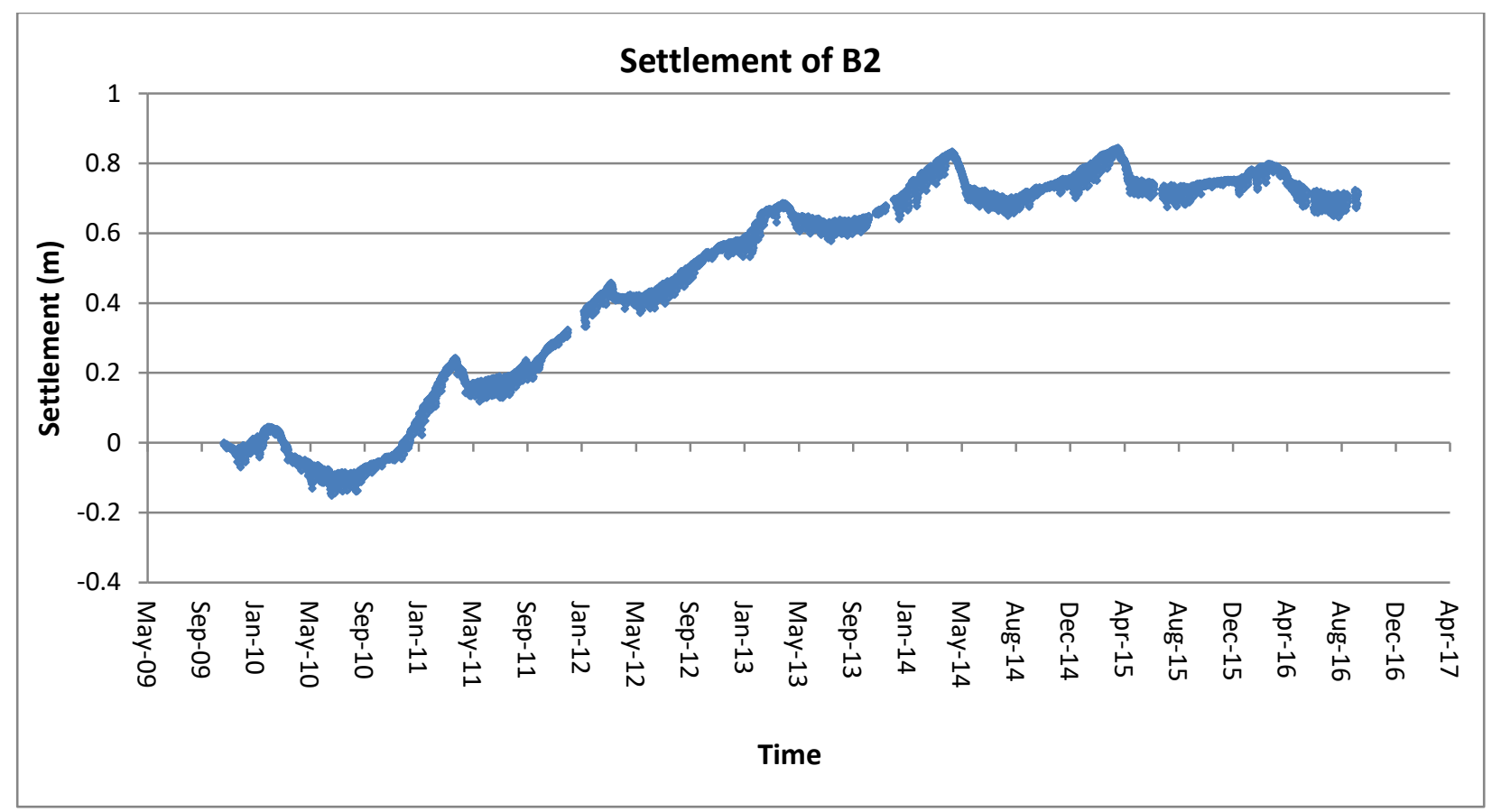

Figure 9: Settlement of Bundle 2

The general trend seen in Figures 8 and 9 shows an increase in settlement due to the placement of overlying waste on top of the clay unit. An apparent seasonal effect is also emergent from the dataset; which shows local peaks in recorded settlement annually circa February or March in a given year. Additionally, there are local lows in the dataset circa July of each year. This fluctuation is speculated to be due to poor temperature correction upon the pressure transducers. The effect of temperature variation on the reference pressures or accumulators in the instrument shed is also suspected. Inspecting the raw settlement data (without B1 or B2 removed) seen in Figures 10-19 shows seasonal trends for Bundles $1-6$, but not so for Bundles $7-12$. The first six bundles are connected to pressure accumulators where the reference pressure is maintained by air in a cylinder. The latter six bundles are connected to a different accumulator where the reference pressure is maintained by a hanging weight on fluid. The air pressure in the cylinder attached to the first six bundles is thought to be varying with temperature and affecting the differential pressure calculated for measuring the settlement. Further investigation on the seasonal variance is ongoing. Because each of the instrument bundles installed in 
the waste profile (B3-B12) are measuring the settlement due to everything beneath them, the bundles' datasets were normalized to the base of the landfill by subtracting the results from Bundles 1 and 2 . This will also allow for a simpler model to be built using a fixed based constraint. The settlement recorded from Bundle 1 was subtracted from all the odd numbered bundles (as they are placed in the same vertical column); likewise, the settlement of Bundle 2 was subtracted from all the even numbered bundles. The settlement of Bundles 3-12 are shown in Figure $10-19$. The 'Fixed Base' results show the corrected settImement results after subtracting B1 or B2; 'With B1' or 'With B2' shows the raw settlement data.

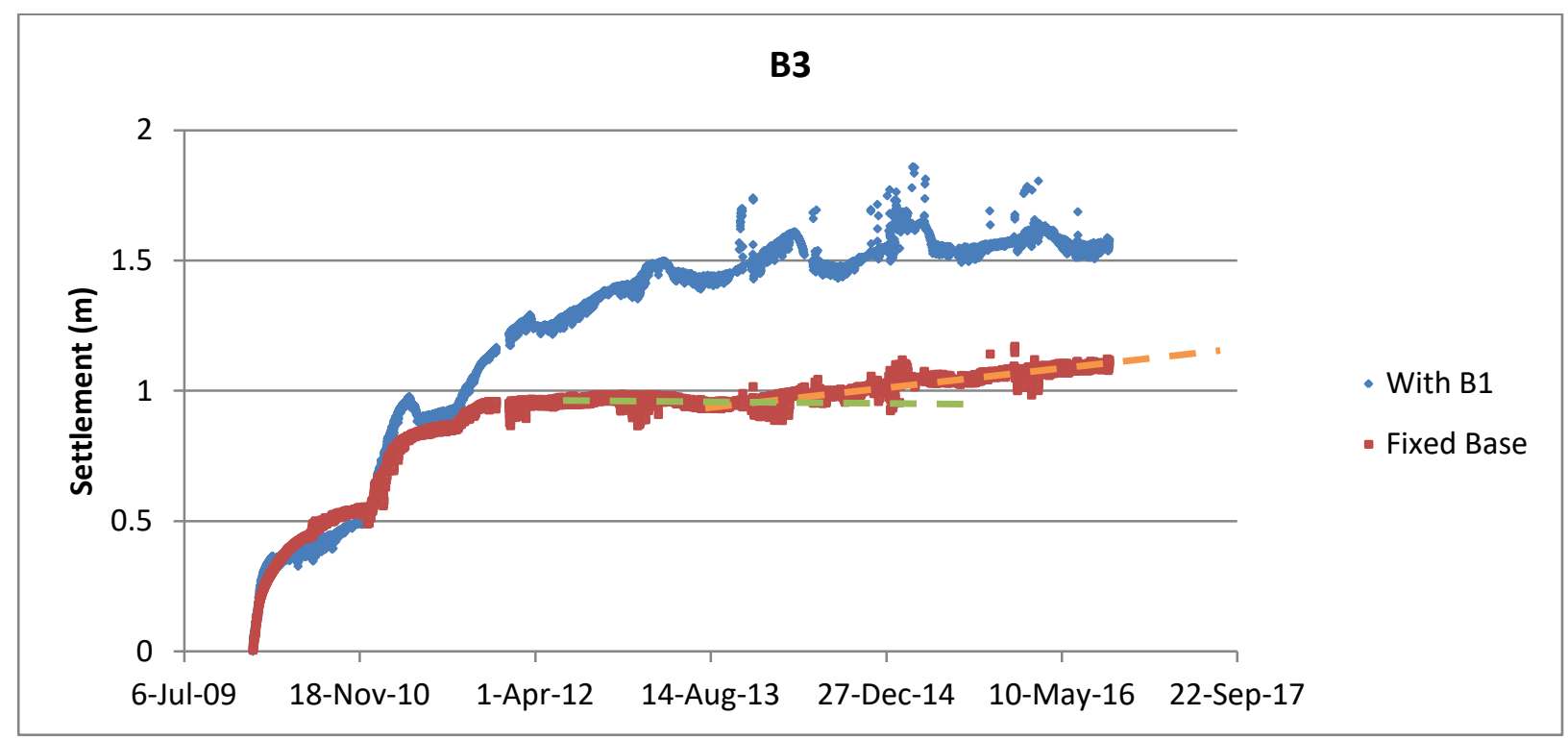

Figure 10: Settlement of Bundle 3. Green line indicates expected settlement rate without temperature effect. Orange line indicates start of new settlement rate due to delayed biodegradation. 


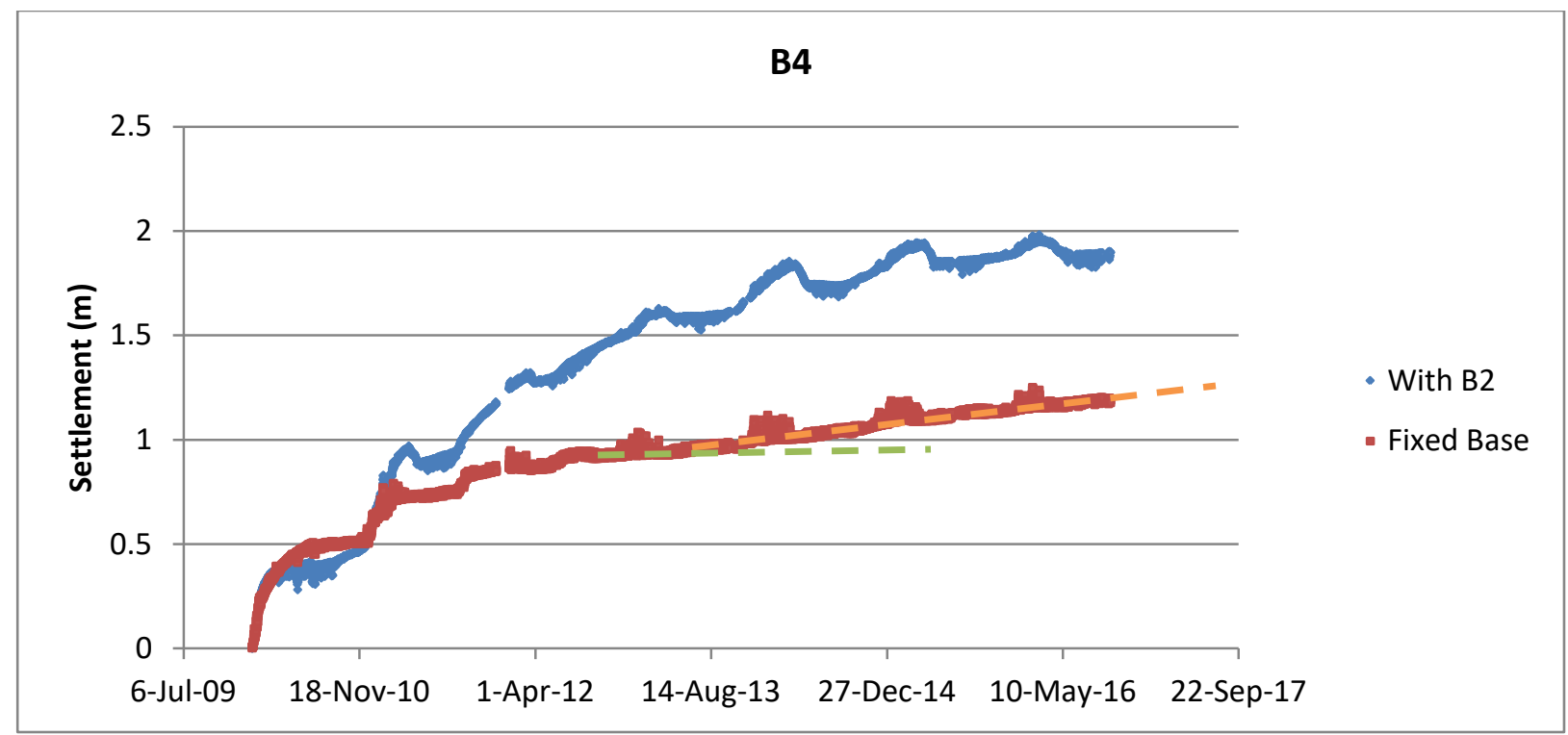

Figure 11: Settlement of Bundle 4. Green line indicates expected settlement rate without temperature effect. Orange line indicates start of new settlement rate due to delayed biodegradation.

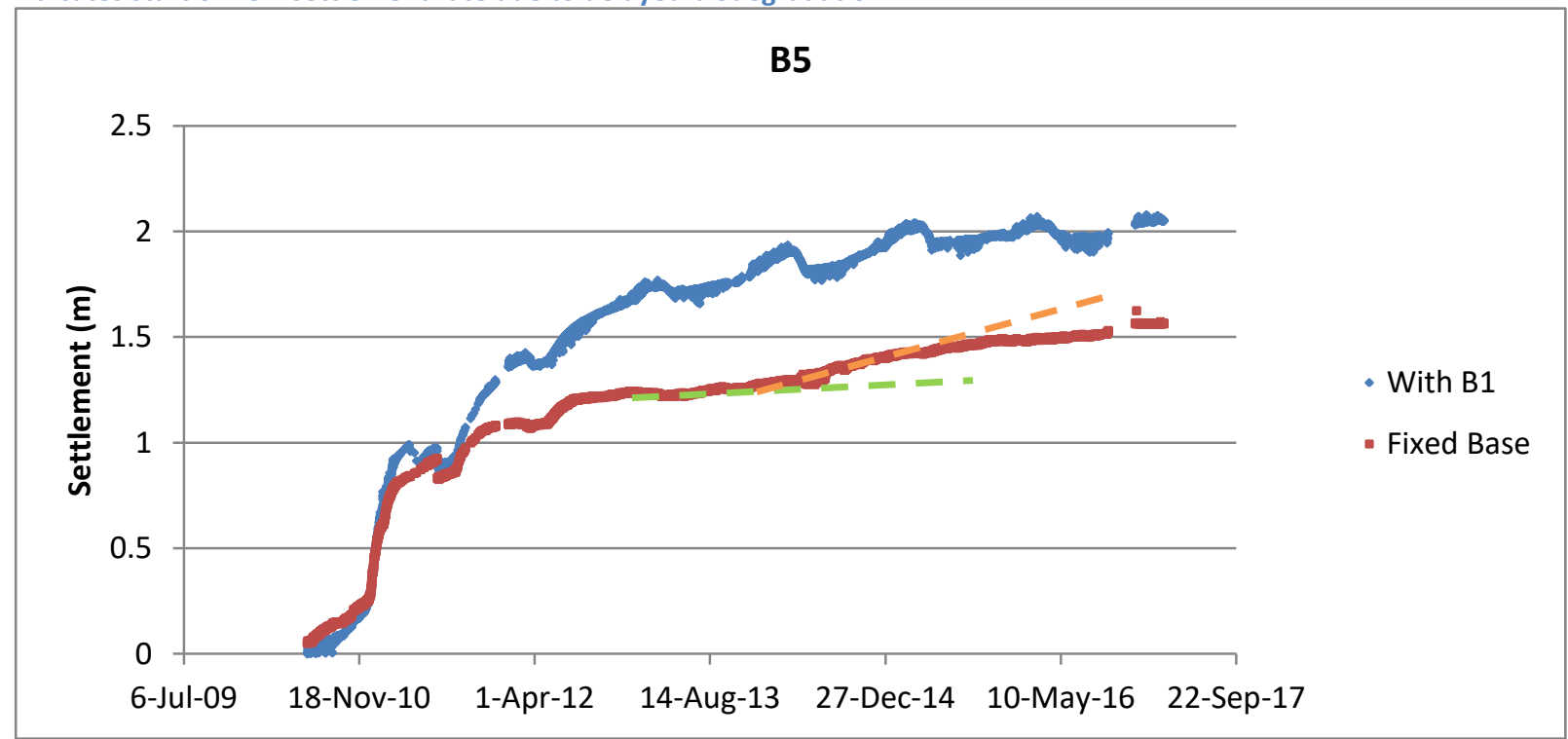

Figure 12: Settlement of Bundle 5. Green line indicates expected settlement rate without temperature effect. Orange line indicates start of new settlement rate due to delayed biodegradation. 


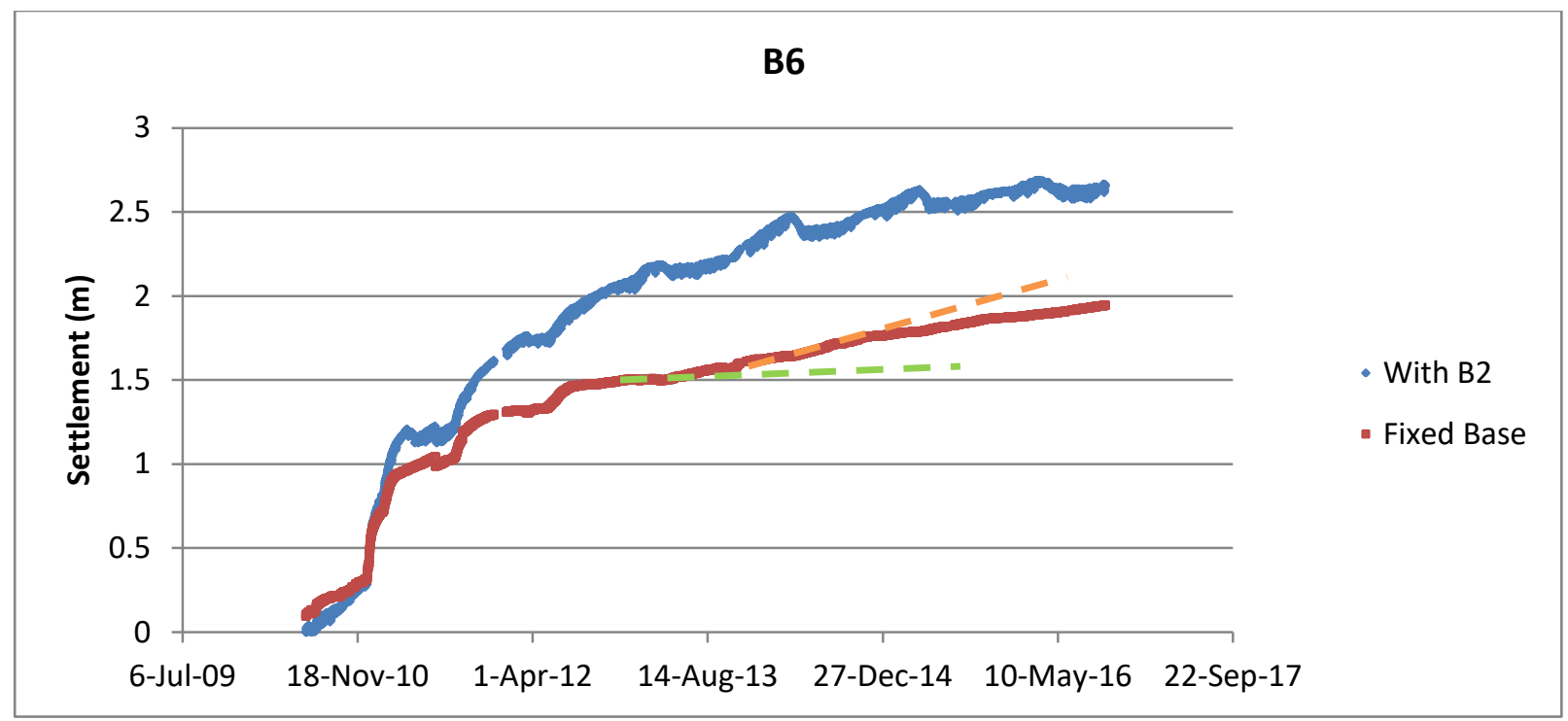

Figure 13: Settlement of Bundle 6. Green line indicates expected settlement rate without temperature effect. Orange line indicates start of new settlement rate due to delayed biodegradation.

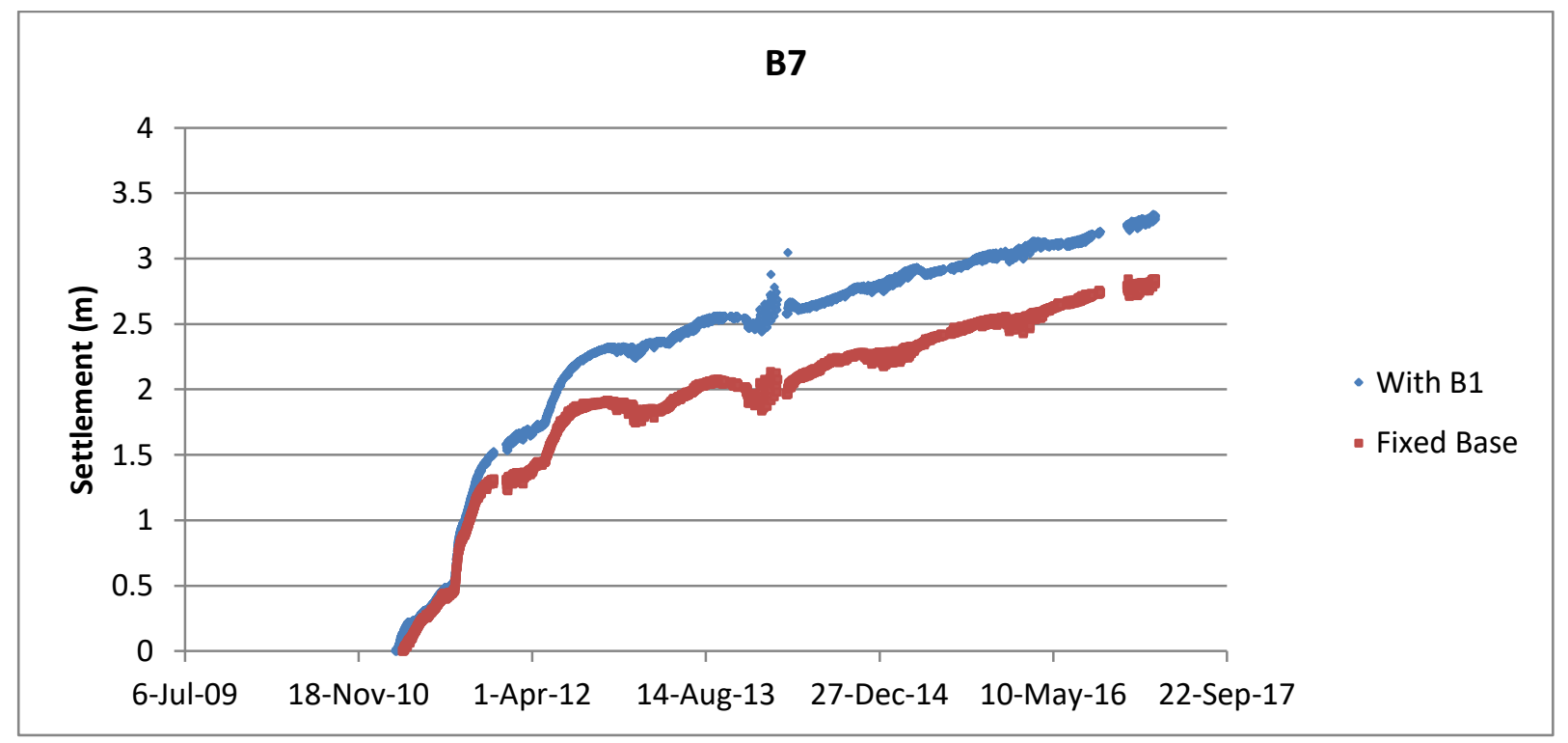

Figure 14: Settlement of Bundle 7 


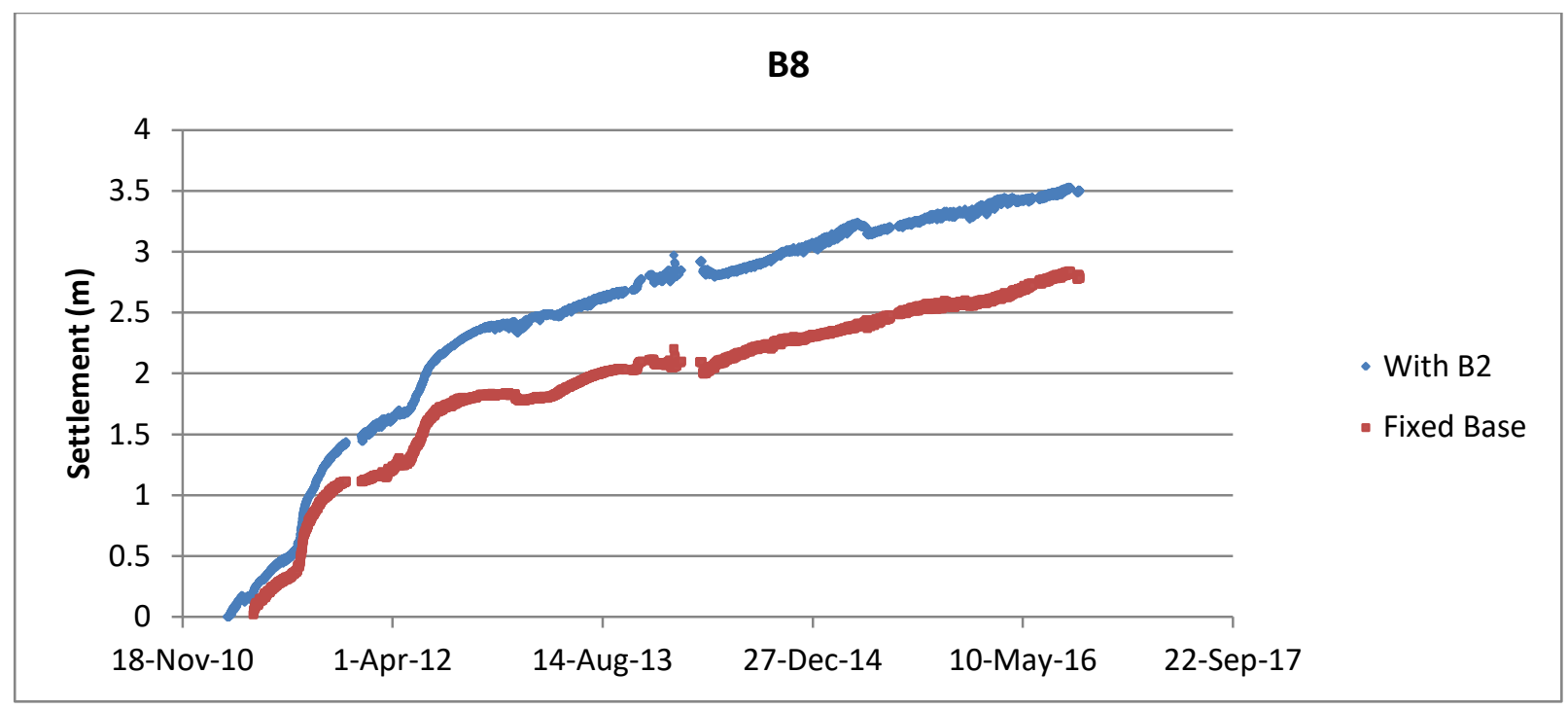

Figure 15: Settlement of Bundle 8

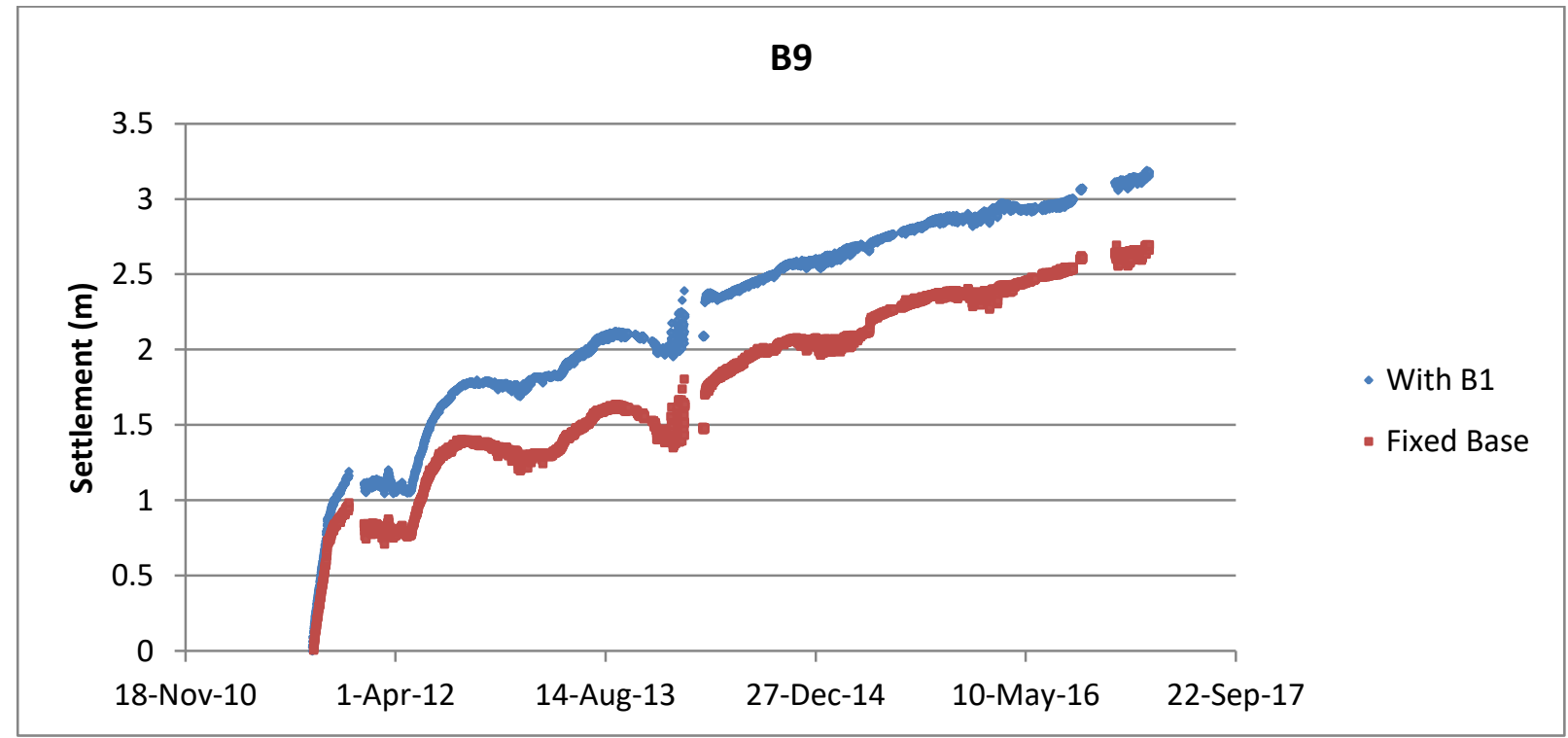

Figure 16: Settlement of Bundle 9 


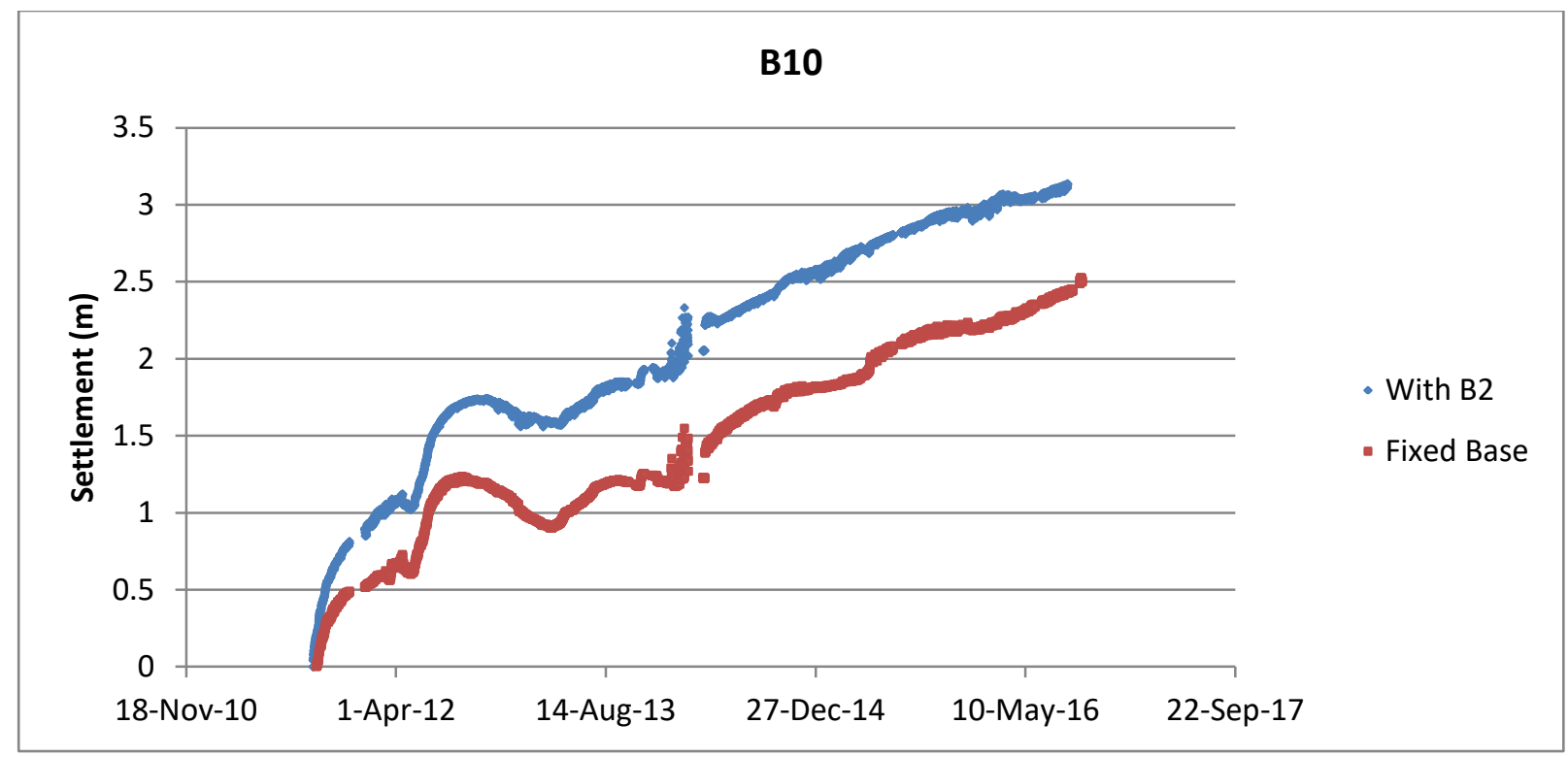

Figure 17: Settlement of Bundle 10

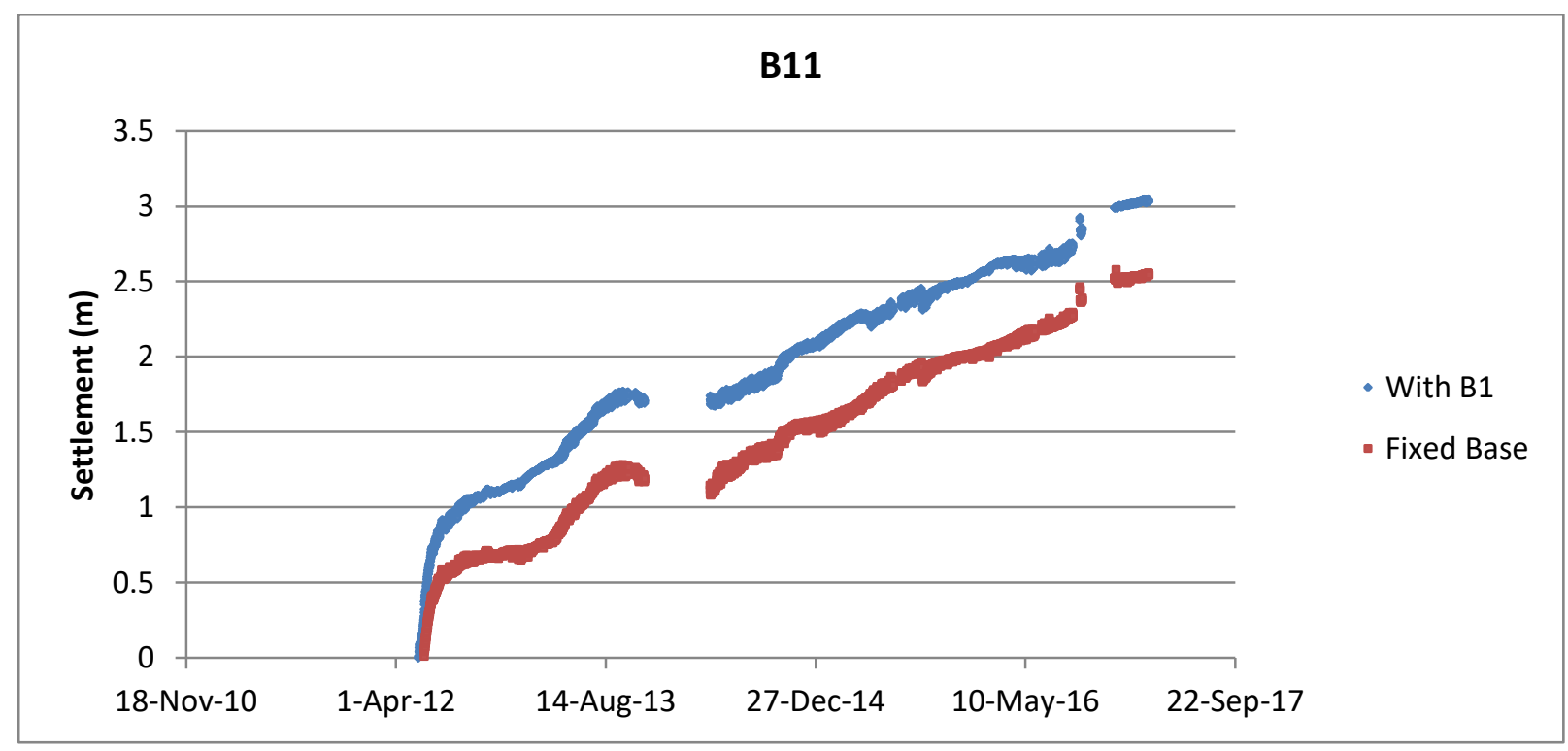

Figure 18: Settlement of Bundle 11 


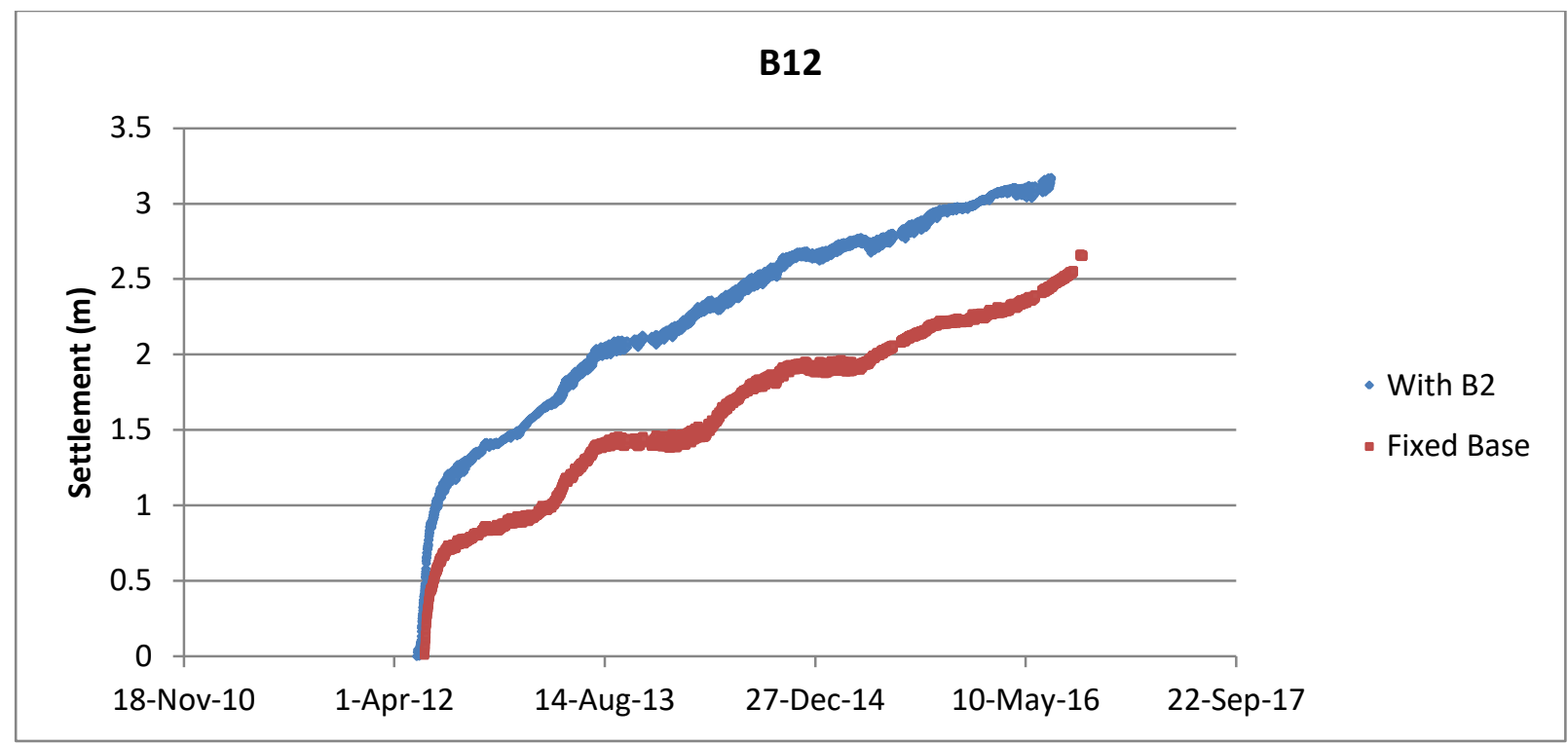

Figure 19: Settlement of Bundle 12

The removal of the underlying clay influence by subtracting Bundles 1 and 2 reduced much of the variability and temperature variance from the data and shows a more favourable pattern of settlement that is common across literature. Inspecting the 'fixed base' plots for Bundles 3, 4, 5, and 6 (Figures 10, 11,12 , and 13) also reveals additional details concerning the active settlement modes. In each bundle's plot, the settlement rate slows after the placement of the fifth waste lift and plateaus before circa August 2013 (see green line). After August 2013, there is an apparent increase in the rate of settlement (see orange line). By correlating the time when the rate of settlement increased with the temperature data recorded (Appendix A), the change in settlement rate beginning in August 2013 is due to the temperature-dependent biodegradation-induced settlement that was delayed due to the bundles being placed in frozen waste. In August 2013, the Bundles 3, 4, 5, and 6 reach a sufficient temperature to allow biodegradation to occur.

Two bundles which did not generally improve after correcting the data set were Bundle 9 and Bundle 10 (Figure 16, 17).These two data sets still feature some pronounced "rebound" behaviour. These rebounds were previously discovered and discussed by Van Geel and Murray (2015) when working with an earlier 
version of the data set. Van Geel and Murray (2015) performed a thorough investigation into the reason for the rebound and deduced that the temperature effects nor the shed's pressure transducer were to blame for the rebound. Additionally, the TEPC recorded constant pressures during the period of rebound. The effect of the rebound remains unknown. One hypothesis considered for the rebound was that the waste placed in the new Zone 5 along the southern slope of Zone 4 caused the uplift of Bundles 9 and 10. Note Bundles 9 and 10 were installed close to the southern slope and the locations of Bundles 11 and 12 were moved further into the landfill to avoid this slope. It could be hypothesized that given the proximity of the southern slope to Bundles 9 and 10, waste placed and compacted in this area may have increased the lateral stress causing the rotation or rebound of Bundles 9 and 10. Lower bundles were not impacted given the greater distance between the southern slope and bundle locations. Bundles 11 and 12 also were not impacted given their locations were moved towards the centre of the landfill to avoid placement so close to the southern slope. For this reason, Van Geel and Murray (2015) considered the compaction or settlement of the waste layer between Bundles 7 and 11 and Bundles 8 and 12 in their analyses.

\section{Finite Element Conceptual Models for Mechanical Settlement}

A more refined mechanical settlement model is proposed to replace the simplistic mechanical component used by Doyle (2015) and to complement the work by Berquist (2017). Two different FEM models are presented in this thesis: one model developed based on the previous work by Marques et al. (2003); and a second model based on the principles of viscoelasticity. Both models were developed in COMSOL Multiphysics, a FEM software package. This software was selected for its functionality in linking mechanical, thermal, and hydraulic processes, which is ideal for future development of this work. Both the Marques et al. (2003) model and the general viscoelastic model were implemented into the same base model, with identical geometry and boundary conditions, but were customized with their unique 
constitutive relations and parameters. Both models feature prescribed 1-D settlement behaviour, but in a 2-D geometry, which features some 2-D loading as a result. Both models idealized MSW as a linear elastic material with time dependent strain contributions.

\subsection{Base Model Setup}

The model geometry was built in 2-D to replicate a cross-section of the Ste. Sophie landfill, but can be easily modified to apply to other landfill sites. A 3-D analysis using a Boussinesq formulation (Boussinesq, 1885) was performed to assure the applicability of a 2-D geometry (Appendix B). The height of the model is determined by the total height of all waste lifts placed. The lower boundary of the model is fixed for all displacement, the top boundary is free to displace, and the side boundaries are subjected to vertical displacement only. The model was built with all waste lifts initially defined and present within the model, but not always active. The model geometry was also discretized into various sections to apply different time-dependent behaviour to different portions of waste, specifically sections defined by the waste lifts and overlapping sections defined by the instrument bundles. The sections defined by the instrument bundles were discretized as layers centered around the instrument bundle locations, i.e. layers with each instrument bundle at its midpoint. These instrument bundle layers will allow behaviourmatching for the area around each instrument bundle, instead of each lift. These bundle layers are used in the FEM model specifically for the biodegradation behaviour.

Data probes are situated in the FEM model at the same estimated heights as the instrument bundles. Some small height adjustment of the probes is involved to account for the movement of the inactive waste lifts placed on top of the active lifts. These probes are used to output the results of the FEM model at the same relative location of the field instruments in the real landfill. The geometry of the model is sufficiently wide, with a sufficient finite element meshing, with a sufficiently small time-step 
used, so these probes will not experience influence from the side boundaries. The probes can be seen in Figure 20.

\subsection{Model Geometry}

The FEM model geometry can be seen in Figure 20. The width of the model is $36.75 \mathrm{~m}$, allows for both columns of instrument bundles in the same model (which are $21.75 \mathrm{~m}$ apart), with an additional $5 \mathrm{~m}$ on the left side and $10 \mathrm{~m}$ on the right side to prevent influence from the boundary conditions. The additional $5 \mathrm{~m}$ on the right side is to allot for the placement of B12, which is placed an additional $5 \mathrm{~m}$ towards the middle of the landfill (i.e. towards right side of the FEM model). The top of the model is sloped to match the measured top slope found overtop the instrument bundles in the field; approximately $11 \%$. The height of the model is determined by the lift heights (Figure 16). Van Geel and Murray (2015) used recorded elevations from the placement of each instrument bundle and their settlement over time to determine the exact height of each waste lift. However, this methodology did not account for the instantaneous settlement of the waste lift itself which occurs during placement of the waste lift. Since the full component of instantaneous effect is accounted for in the FEM model, the lift heights were modified to account for their "true" unsettled state. This instantaneous portion during the first few weeks of placement was found to account for approximately $1.25 \%$ of the lift height, and as such, the lift heights used in the model were increased from the heights from Berquist (2017) by $1.25 \%$ to compensate. The model was meshed using a Lagrangian mesh. The mesh uses full integrated, quadrilateral elements in a mapped sequence for the entire waste profile. The size of the mesh was prescribed as 'fine' with a maximum element size of $1.95 \mathrm{~m}$ and a minimum size of $0.011 \mathrm{~m}$. The mesh element quality was measured for 'volume versus length' and 'skewness'. Mesh element quality ranges from 0 to 1, with 1 representing a perfectly regular element, and 0 being a degenerated element. Average element quality achieved for 'volume versus length' and 'skewness' were good at 0.9172 and 
0.9732 respectively. A fine mesh with good element quality will ensure higher model accuracy, homogeneous settlement, and good convergence. The meshing results can be seen in Figure 21 .

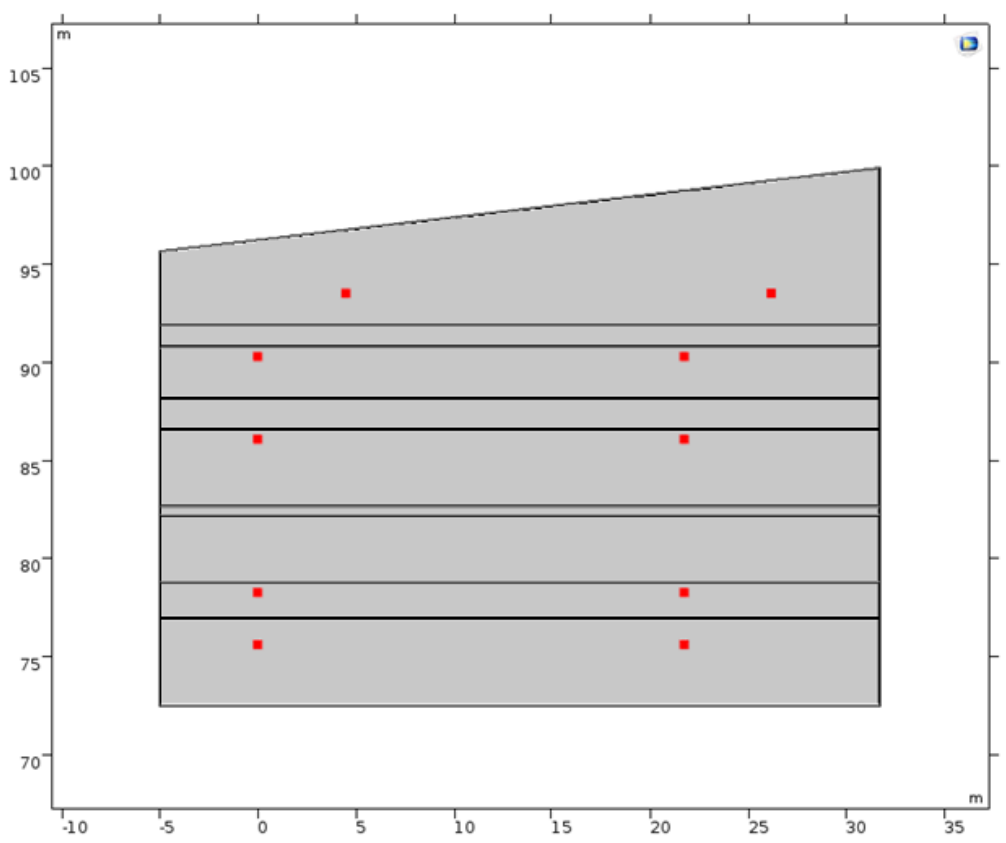

Figure 20:FEM Model Geometry with Probes

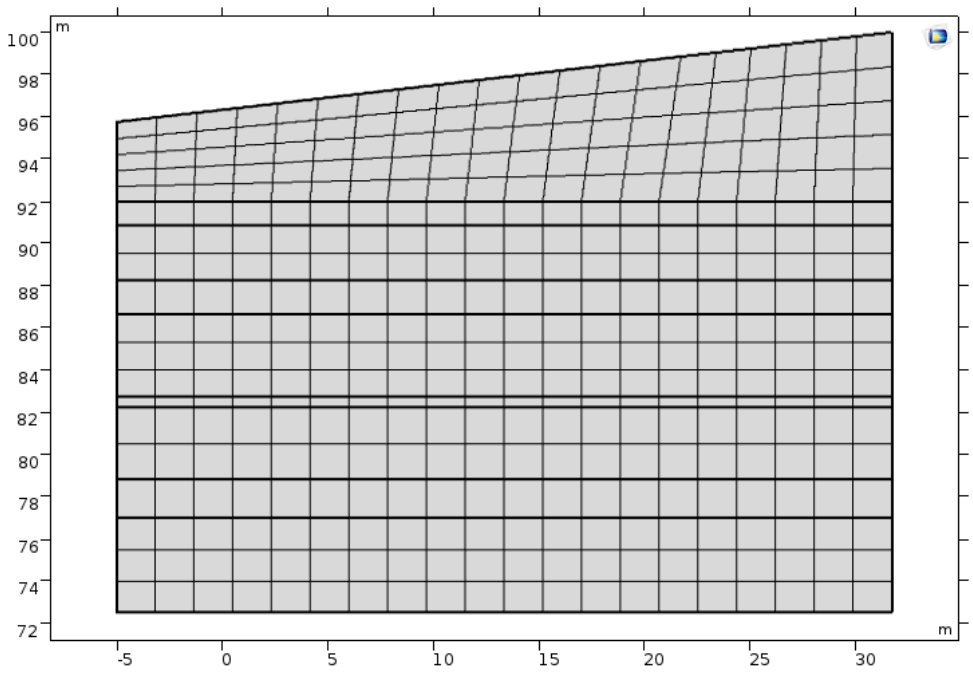

Figure 21: FEM Model Meshing 


\subsection{Construction-Equivalent Loading}

The applied loading in the model is handled by a body force on each waste lift to simulate the effect of gravity on the waste. The construction process of the landfill will be simulated by activating this gravitational force to each successive waste lift over time. The gravitational effect at each lift will be turned on over a short time interval to replicate the construction time of each lift (14 days). The gravitational force on a waste lift will start at a zero magnitude and progressively increase until it reaches its full magnitude $(9.81 \mathrm{~N} / \mathrm{kg})$ at the end of the 14 day interval. After 14 days, the gravitational force will remain at full magnitude for the remainder of the simulation. This methodology allows all waste lifts to exist during the entire model simulation, while allowing waste lifts to be inactive until such a time when a waste lift is "placed".

\subsection{Instantaneous Behaviour}

In the Marques version of the FEM model, the instantaneous response due to loading is handled by the linear elastic response of the finite elements themselves. In the viscoelastic version of the model, this same behaviour is present, but it is coupled with the viscoelastic response (which is discussed Section 4.5). The original incarnation of the viscoelastic model in McDonald et al. (2017) used a three-parameter solid for the viscoelastic body, which has now been replaced with a single Kelvin-Voight body. During McDonald et al. (2017)'s parametric optimization of the three-parameter solid to field data, the parameters controlling the initial linear spring became increasingly insignificant as the optimization approached better data matching. The optimization suggested the initial spring was unnecessary and was subsequently removed in favour of implementing a lone KV body. Overall, the time-dependent response was more dominant and allowed a better fit to the data.

In COMSOL Multiphysics, the MSW is idealized as a linear elastic material with stress-dependent parameters. Even though MSW is highly variable from layer to layer, the material as it exists in the FEM 
computation is an isotropic material. Due to the majority of settlement mechanisms being investigated are one dimensional in nature, the implementation of isotropy was deemed fair.

In COMSOL, the response of the instantaneous behaviour is controlled by the elasticity matrix, which dictates the stiffness of the elastic material. The elasticity matrix is defined by any two elastic material parameters; such as Young's modulus, Poisson's ratio, Shear Modulus, Bulk Modulus, Lamé parameters, pressure-wave speed, or shear-wave speed. In this model, Young's modulus and Poisson's ratio are used to define the elasticity matrix as they are more commonly reported in literature concerning MSW (or similar material) properties. The elasticity matrix is shown in Equation 4-A.

Equation 4-A

$$
D=\frac{E}{(1+v)(1-2 v)}\left[\begin{array}{cccccc}
1-v & v & v & 0 & 0 & 0 \\
v & 1-v & v & 0 & 0 & 0 \\
v & v & 1-v & 0 & 0 & 0 \\
0 & 0 & 0 & \frac{1-2 v}{2} & 0 & 0 \\
0 & 0 & 0 & 0 & \frac{1-2 v}{2} & 0 \\
0 & 0 & 0 & 0 & 0 & \frac{1-2 v}{2}
\end{array}\right]
$$

where $D$ is the elasticity matrix

$E$ is the Young's Modulus

$v$ is the Poisson's ratio

To better match the displacement data recorded in-situ at the Ste. Sophie Landfill, the elastic material behaviour was modified to change with increased loading. This was achieved by making the elastic material parameters, Young's modulus and Poisson's ratio, stress/strain-dependent.

Young's modulus for MSW is thought to behave similar to soils, and should increase with depth and confining stress. This theory is consistent with large scale compression cell tests by Beaven and Powrie 
(1995) and by Singh and Fleming (2008a). To implement this effect, a modified version of the power function proposed by Janbu (1963) was selected (Equation 4-B). The modification made to Janbu's original equation is the addition of a "starting Young's Modulus", $E_{\text {start }}$. Typically, Young's modulus is thought to approach zero with zero confining stress, which is consistent with laboratory compression cell data (Singh and Fleming, 2008a). However in the field, after placement, waste undergoes compaction under heavy machinery as part of regular landfill operations. It is thought that this loading and unloading would leave a residual stiffness in the waste and thus a starting Young's modulus above zero. This initial compaction behaviour is not present in the FEM model due to insufficient data to implement a correct loading sequence at the appropriate time to be in accordance with the field data. For this reason, a starting value of Young's Modulus is included to account for the compaction of the waste in the field. Janbu (1963) shows values of ' $\mathrm{N}=0$ ' for solid rock, ' $\mathrm{N}=0.5$ ' for aggregates, and ' $\mathrm{N}=1$ ' for wet clay. To match the trends seen in Beaven and Powrie (1995) and by Singh and Fleming (2008a) with Equation 4-B, the fitting parameter ' $\mathrm{N}$ ' is expected to have a value between 1 and 0.5 for MSW. These limits are for a comparable medium that ranges between an aggregate and a wet clay.

Equation 4-B

$$
E=E_{\text {start }}+M * P_{A T M}\left(\frac{\sigma_{y}}{P_{A T M}}\right)^{N}
$$

where $E_{\text {start }}$ is the predefined starting value of Young's Modulus ( $\left.\mathrm{kPa}\right)$

$$
\begin{aligned}
& M, N \text { are fitting parameters } \\
& P_{A T M} \text { is atmospheric pressure }(=101.3 \mathrm{kPa}) \\
& \sigma_{y} \text { is the vertical stress }(\mathrm{kPa})
\end{aligned}
$$


A similar modification was made for the elastic material's Poisson's ratio. Ramaiah and Ramana (2017) used large-scale triaxial tests to study the stress-strain volume change behavior of MSW. Their findings show Poisson's ratio varying with axial strain for different specimens of MSW with varying fiber content and a local soil. To replicate this strain-dependency in the FEM models, the Poisson's ratio is now defined by Equation 4-C. Incorporating this strain-dependency into the FEM model will also connect the change in elastic material properties with the time-dependent strain effects from mechanical creep and biodegradation-induced strain. The equations features two fitting parameters, $A$ and $B$, and a starting value of 0.1 for zero axial strain. This starting value could differ depending on the true fiber content, but 0.1 is a moderate compromise and achieves a good fit. The fitting parameter ' $\mathrm{B}$ ' should be close to 1 to match the trends in Ramaiah and Ramana (2017) and produce a near-linear fit.

Equation 4-C

$$
v=0.1+A * a b s\left(\varepsilon_{\text {axial }}\right)^{B}
$$

where $A, B$ are fitting parameters

$\varepsilon_{\text {axial }}$ is the axial strain in the material

\subsection{Time-Dependent Strain}

Municipal solid waste features two main contributors to time-dependent strain: mechanical creep and biodegradation-induced strain. Separating these two mechanisms is a known difficulty across many models published in literature, as underestimating or overestimating one of the contributors can drastically affect the long-term behaviour. Proper separation typically requires more detailed field data, refined settlement behaviour, or both. 


\subsubsection{Mechanical Creep}

\subsubsection{Marques et al. (2003) Model}

The model proposed by Marques et al. (2003) included three distinct explicit expressions for the three mechanisms of settlement for MSW : instantaneous compression, biodegradation-induced settlement, and mechanical creep. The expression used by that model for mechanical creep can be seen in Equation 2-H. For implementation of this equation in COMSOL, the first derivative of Equation 2-H was taken and included as creep strain rate (see Equation 4-D).

Equation 4-D

$$
\frac{\partial \varepsilon_{C}}{\partial t^{\prime}}=b(\Delta \sigma)(c)\left(e^{-c t^{\prime}}\right)
$$

This creep strain rate is applied to each waste lift in the model and is activated using the same approach to mobilize gravitational forces. The creep strain is only effective after the waste lift is "placed" and becomes active, and then remains active for the rest of the simulation. The stress term in this equation is taken as the total vertical stress in COMSOL, which may change throughout the simulation due to the placement of additional overlying lifts.

\subsubsection{Viscoelastic Model}

In contrast with the explicit creep equation used in the Marques et al. (2003) approach, the timedependent response for mechanical creep will be idealized as a linear viscoelastic response. This linear viscoelastic approach uses an additive decomposition of stresses and strains and assumes the volumetric deformation to be elastic. The specific viscoelastic model being used in this computation is a single Kelvin-Voight viscoelastic body. This model can be represented by a spring in parallel with a dashpot. The spring seen in the model is the linear elastic material response of the FEM material, which in this case is synonymous with what was previously noted as the 'instantaneous response to loading'. 
This instantaneous response (with its stress-dependent features) is now coupled with a viscous dashpot to produce a nonlinear, time dependent viscoelastic response.

The shear modulus, $G$, defines the stiffness of the spring and is derived from the two other elastic material properties, Young's Modulus, $E$, and Poisson's ratio, $v$. The computation of $G$ from $E$ and $v$ can be seen in Equation 4-E. The stress tensor of the viscoelastic response is calculated from the elastic strain rate (Equation 4-F), and $\tau$ represents the relaxation time of the dashpot, which in a rheological sense, is the time it takes for the dashpot to dissipate. The dashpot relaxation time can be considered the effective time span of the mechanical creep response. The relationship between viscosity and relaxation time is shown in Equation 4-G.

Equation 4-E

$$
G=\frac{E}{2(1+v)}
$$

Equation 4-F

$$
\sigma_{q}=2 \eta \dot{\varepsilon}=2 G \tau \dot{\varepsilon}
$$

Equation 4-G

$$
\eta=G \tau
$$

where $G$ is the shear modulus

$\sigma_{q}$ is the stress in the viscoelastic response

$\eta$ is the viscosity

$\tau$ is the relaxation time of the dashpot

$\dot{\varepsilon}$ is the elastic strain rate 


\subsubsection{Biodegradation-Induced Strain}

Unlike the mechanical creep behaviour, the same component for calculating biodegradation-induced strain is included in both the Marques version and the viscoelastic version of the FEM model. Marques at al. (2003) uses an exponential relationship for biodegradation-induced strain that was previously proposed by Park and Lee (1997). The expression assumed biodegradation-induced settlement starts immediately after the waste is placed and is not temperature dependent. Using the exponential relationship from Marques et al. (2003) for biodegradation-induced settlement would not accurately reflect the true biodegradation behaviour and the time it takes for the waste placed under frozen conditions to reach a sufficient temperature $\left(\operatorname{circa} 10^{\circ} \mathrm{C}\right)$ to support biodegradation. This temperature dependency indicates that for MSW placed in cold temperatures, the biodegradation effect does not occur immediately after lift placement, such as in Marques et al. (2003), but is a delayed response dependent on the thermal profile of the waste.

Temperature data from Ste. Sophie indicated that waste placed during the winter season can take months or years before the MSW's temperature approached ideal levels for anaerobic biodegradation to occur. This behaviour can be seen clearly in the 'fixed base' plots for Bundles 3, 4, 5, and 6 (Figures 10, 11,12 , and 13). In each bundle's plot, the settlement behaviour slows after the placement of the fifth waste lift and plateaus before circa August 2013. After August 2013, there is an apparent increase in the rate of settlement. By correlating this settlement pattern with the temperature data recorded (Appendix A), the settlement beginning in August 2013 is the temperature-dependent biodegradation-induced settlement that was delayed due to the bundles being placed in frozen waste. In August 2013, Bundles $3,4,5$, and 6 reach a sufficient temperature to allow biodegradation to occur.

Without a temperature-dependency, the biodegradation-induced settlement would also be difficult to distinguish from the mechanical creep-induced settlement, as both terms are depicted by exponential 
curves in Marques et al. (2003). For these reasons, the biodegradation term featured in Marques et al. (2003) was replaced by a temperature-dependent biodegradation-induced settlement term proposed by Berquist (2017).

Berquist (2017) in her thermal model simulated the temperature changes and expended energy (heat) due to anaerobic biodegradation using a model proposed by Hanson et al. (2013). Berquist's model tracked the energy expended with time for the Ste. Sophie site. The biodegradation-induced settlement with time was a function of the expended energy relative to the total expended energy; i.e. if $50 \%$ of the energy has been expended, then $50 \%$ of the biodegradation-induced settlement has occurred. The simulation expressed as the fraction of expended energy over the total expended energy with time, are shown in Figure 22 and 23. To incorporate temperature-dependent biodegradation-induced settlement term in the current model, a cumulative gamma function was fit to the data presented in Figure 22 and 23. Figure 22 shows the biodegradation behaviour up to Year 7, which is the time-span used in the current model`s results. Figure 23 shows the continuation of the biodegradation behaviour past the model results up to 13 years. Both the proposed viscoelastic model and the Marques et al. (2003) model use these cumulative gamma distribution functions to approximate biodegradation-induced strain at the various bundle locations.

Multiplying this gamma function by $E_{D G}$, the total amount of strain that can occur due to biological decomposition, the curves represent the biodegradation-induced strain over time at the instrument bundle locations. The differences in these curves stem from the presence of frozen waste, the time of bundle installation, and temperature variations throughout the waste lifts placed in the landfill. Bundle 5, for example, was placed at the top of the first waste lift in June. Being close to the top of a waste lift, which was not covered by any other lifts at this point, B5's temperature lagged the seasonal ambient temperature. Being placed in warm conditions, the waste surrounding B5 began to slowly biodegrade. 
This biodegradation slowed and eventually stopped when the climate changed to colder temperatures which would not permit biological activity. The second waste lift was placed during this period of cold temperatures, which covered and insulated B5 at a colder temperature, causing the plotted plateau in Figures 22 and 23, until the surrounding waste achieved a warmer temperature.

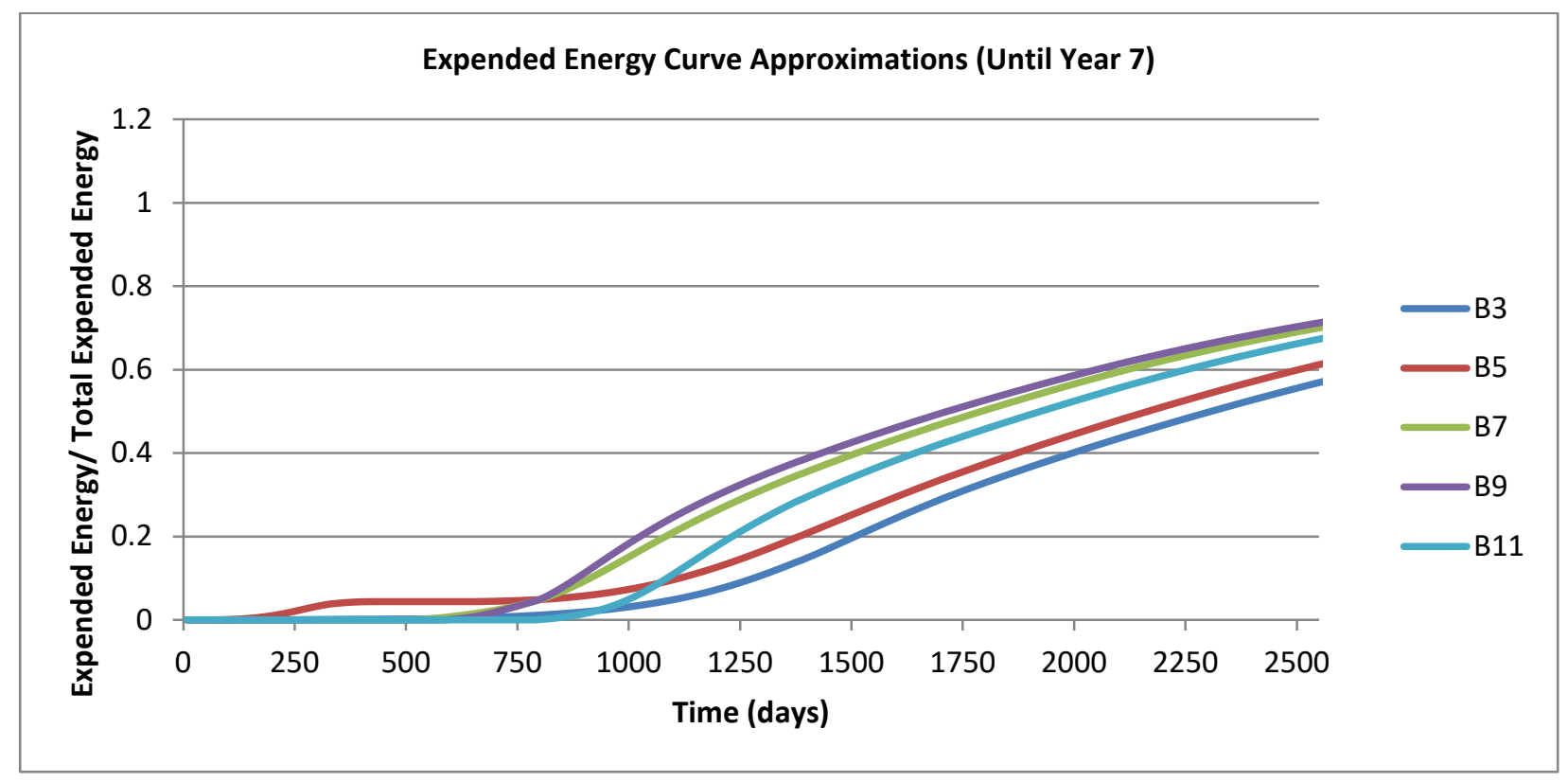

Figure 22: Cumulative Gamma Distribution Function for Expended Energy Curve Approximation 


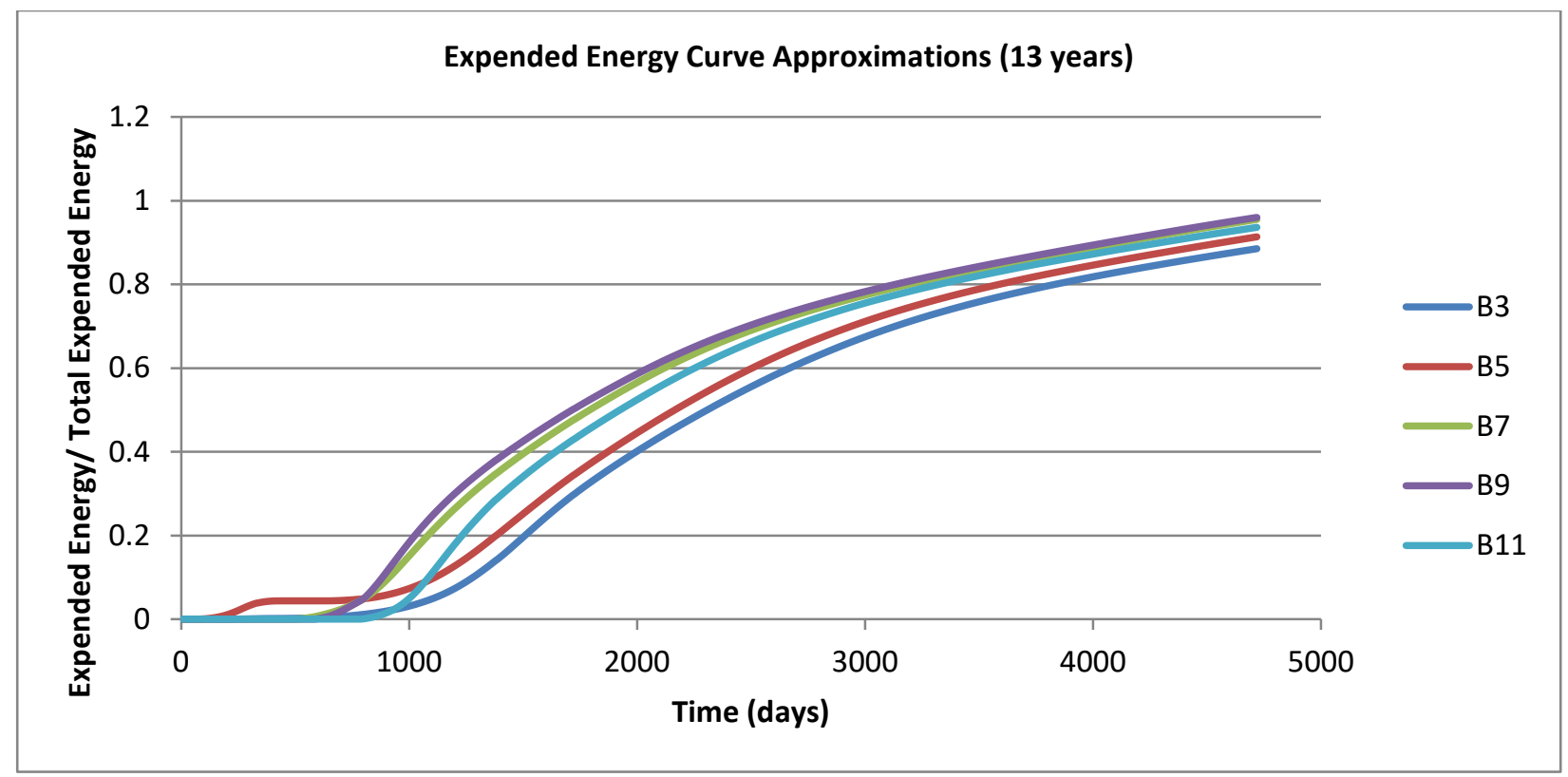

Figure 23: Cumulative Gamma Distribution Function for Expended Energy Curve Approximation

Each of these curves was inputted into COMSOL Multiphysics as an interpolated function. Each curve describes the biodegradation activity in the area surrounding each instrument bundle. Because each bundle does not align perfectly with a waste lift, the model geometry was further discretized into bundle layers; layers created in the waste profile by having each instrument bundle as its midpoint. Each bundle layer then receives additional strain as dictated by its respective curve multiplied by $\mathrm{E}_{\mathrm{DG}}$. Only one set of energy curves are used in this model. This means the odd bundle curves seen in Figures 22 and 27 control the biodegradation behaviour surrounding the even bundles as well. The odd bundles also control the bundle layer locations in the cross-section of the waste.

\section{Mechanical Model Optimization, Results, and Discussion}

The Marques-based model and the viscoelastic model both underwent a multiple-objective least squares objective optimization to solve for optimal control parameters. The optimization was done within COMSOL Multiphysics using a Nelder-Mead method based solver. The optimization was 
performed to match the field settlement data to the results simulated in COMSOL. The field data used in the optimization was the difference between the vertical spatial coordinate of the instrument bundles' installation $(\mathrm{Y})$, and the vertical displacement (v) relative to the aforementioned spatial coordinate. Each mechanical compressibility model was optimized to three sets of field data; the first set contained the displacement data for Bundle 3, the second set contained the displacement data for Bundle 4, and the third set contained the displacement data for both Bundle 3 and Bundle 4. Notably, these three field displacement sets are corrected to a "fixed-base" condition by removing the settlement of Bundle 1 and Bundle 2 for the odd and even bundle sets respectively. This fixed-base condition is discussed in Section 3 and shown in Figures $10-19$.

All optimization runs provided a good match to the field data, i.e. Bundles 3 and 4 , and good prediction of the upper bundles, i.e. Bundles 5-12. As was previously discussed in Section 3, the field data for Bundles 9 and 10 displayed results which illustrated they may have been impacted by waste placement in Zone 5. Because of this discrepancy, the results of Bundle 9 and Bundle 10 are included in the following results, but will not be considered in discussions of fitting or data matching.

The results presented in the following subsections compare the FEM model predictions with field data. Data probes were positioned in the FEM model to monitor settlement-time relationships within the bundle layer for comparison with the field data. Because the probes are omnipresent throughout the entire simulation, the probes record displacement before the 'true' start (i.e. installation date) of the instrument bundles. This is due to the simulated settlement of the active waste layers below the probes and the corresponding movement of the mesh due to this simulated settlement. Given this, it was difficult to correctly identify an accurate initial probe elevation such that when the waste surrounding the probe was placed (i.e. the gravity term turned on), the probe elevation was the same as the actual field elevation. Therefore the probe data were adjusted to synchronize the predicted settlement with 
the measured field data by adjusting the height of the trend line of the probe data by a linear shift such that the elevation change overlayed the field data elevations, which was the basis for the parameter optimization within the numerical modelling procedures. Significance of the probe data offset can be seen through comparison of Figures 24 with Figure 25, which corresponds to before and after the probe data calibration respectively. Table 2 shows the magnitude of the probe data adjustment for each optimization run. The probes for B3 and B4 did not require any adjustment due to their installation near the start of the simulation, as the probes did not experience any significant displacement before their 'true installation'. Upper bundles required larger values of shifting due to the larger amount of underlying waste beneath the probe undergoing compression, and the larger amount of time passed before 'true installation'.

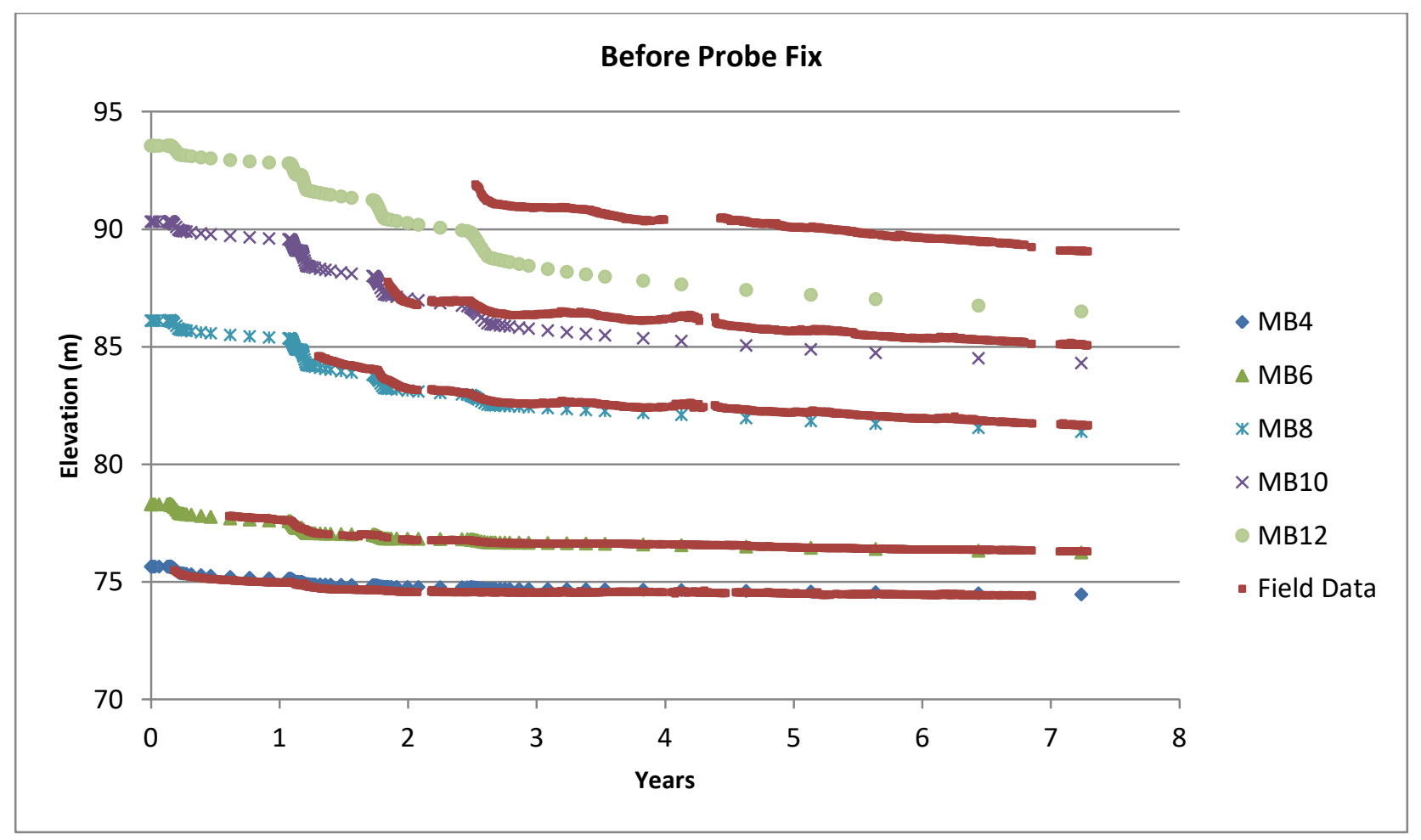

Figure 24: Marques Model results (MB\#) compared to field data before data probe location fix 


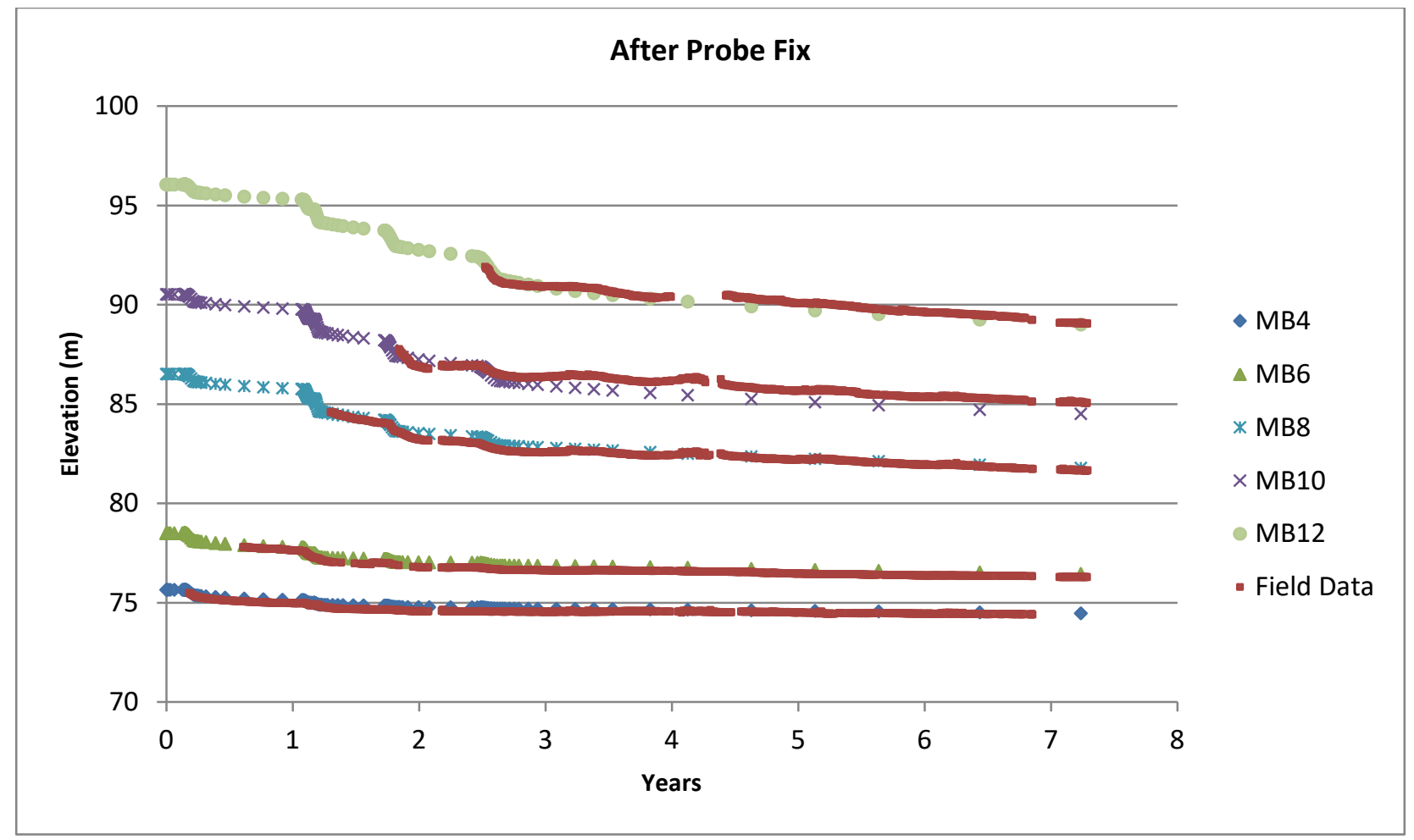

Figure 25: Marques Model results (MB\#) compared to field data after data probe location fix

Table 2: Data Probe Adjustments per bundle, per optimization run

\begin{tabular}{|c|c|c|c|c|c|}
\hline & B3 & B5 & B7 & B9 & B11 \\
\hline $\begin{array}{l}\text { Marques } \\
\text { (B3+B4) }\end{array}$ & 0 & $+0.1 \mathrm{~m}$ & $+0.4 \mathrm{~m}$ & $+0.2 \mathrm{~m}$ & $+2.5 \mathrm{~m}$ \\
\hline $\begin{array}{l}\text { Marques } \\
\text { (B3) }\end{array}$ & 0 & $+0.1 \mathrm{~m}$ & $+0.4 \mathrm{~m}$ & $+0.2 \mathrm{~m}$ & $+2.7 \mathrm{~m}$ \\
\hline $\begin{array}{l}\text { Viscoelastic } \\
\text { (B3+B4) }\end{array}$ & 0 & $+0.2 \mathrm{~m}$ & $+0.6 \mathrm{~m}$ & $+0.3 \mathrm{~m}$ & $+3.2 \mathrm{~m}$ \\
\hline \multirow{2}{*}{$\begin{array}{l}\text { Viscoelastic } \\
\text { (B3) }\end{array}$} & 0 & $+0.2 \mathrm{~m}$ & $+0.6 \mathrm{~m}$ & $+0.5 \mathrm{~m}$ & $+3.1 \mathrm{~m}$ \\
\hline & B4 & B6 & B8 & B10 & B12 \\
\hline $\begin{array}{l}\text { Marques } \\
\text { (B3+B4) }\end{array}$ & 0 & $+0.5 \mathrm{~m}$ & $+0.6 \mathrm{~m}$ & $+0.3 \mathrm{~m}$ & $+3 \mathrm{~m}$ \\
\hline $\begin{array}{l}\text { Marques } \\
\text { (B4) }\end{array}$ & 0 & $+0.5 \mathrm{~m}$ & $+0.6 \mathrm{~m}$ & $+0.3 \mathrm{~m}$ & $+3 \mathrm{~m}$ \\
\hline $\begin{array}{l}\text { Viscoelastic } \\
\text { (B3+B4) }\end{array}$ & 0 & $+0.5 \mathrm{~m}$ & $+0.8 \mathrm{~m}$ & $+0.5 \mathrm{~m}$ & $+3.5 \mathrm{~m}$ \\
\hline $\begin{array}{l}\text { Viscoelastic } \\
\text { (B4) }\end{array}$ & 0 & $+0.5 \mathrm{~m}$ & $+0.8 \mathrm{~m}$ & $+0.6 \mathrm{~m}$ & $+3.5 \mathrm{~m}$ \\
\hline
\end{tabular}




\subsection{Marques-based model Results}

Overall, the Marques-based model performed very well. It yielded a very good match with the even bundle field data and a good match with the odd bundle field data. Figures $26-33$ show the plots of the simulated and field settlement based on optimizations using Bundle 3, Bundle 4, and Bundles 3 and 4 together. Figures 26, 28, 30, and 32 show the elevation of all bundles. Figures 27, 29, 31, and 33 show the corresponding simulated and field displacement at the bundles used for optimization. Table 3 shows the achieved best fit parameters from model optimization. The table has an additional row for 'Resulting Fit' - this is the degree of data matching to the input field data by the Nelder-Mead method based solver. A 'Resulting Fit' of zero would indicate a perfect match. The value of the resulting fit does not indicate which parameter set is best, as it does not correlate with the accuracy in predicting the upper bundles, and is dependent on the number of data points input. The resulting fit is only useful as a value for comparing the magnitude of correlation to the field data of the lower bundles for which it is optimized. The table also includes columns for comparing the relative difference of each optimization's parameters to the parameters obtained from B4's optimization. B4's optimization was selected as the reference case because the B4 field data used in optimization was free of any large abnormalities in settlement response. 


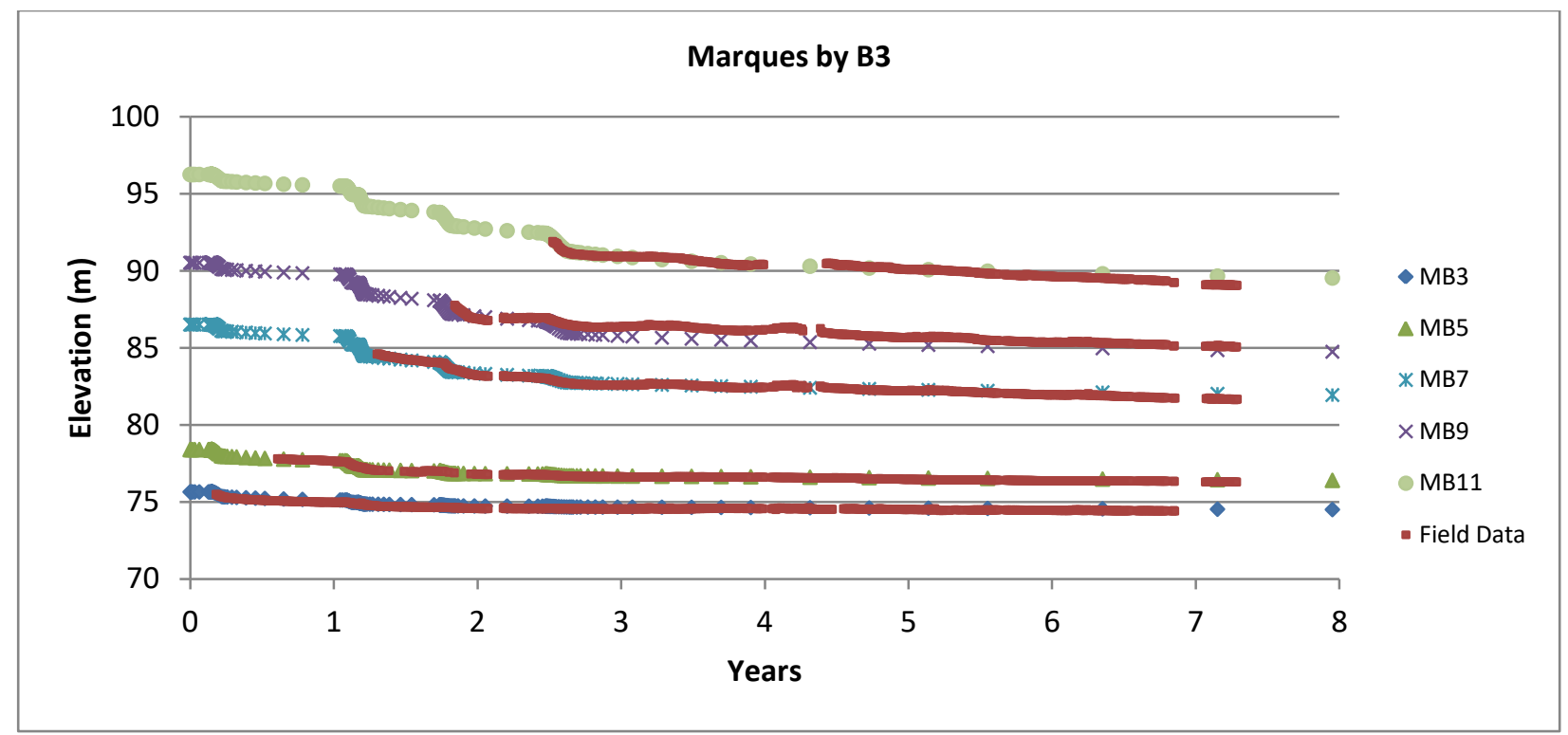

Figure 26: Marques-based model optimized to B3, elevation of odd numbered bundles results

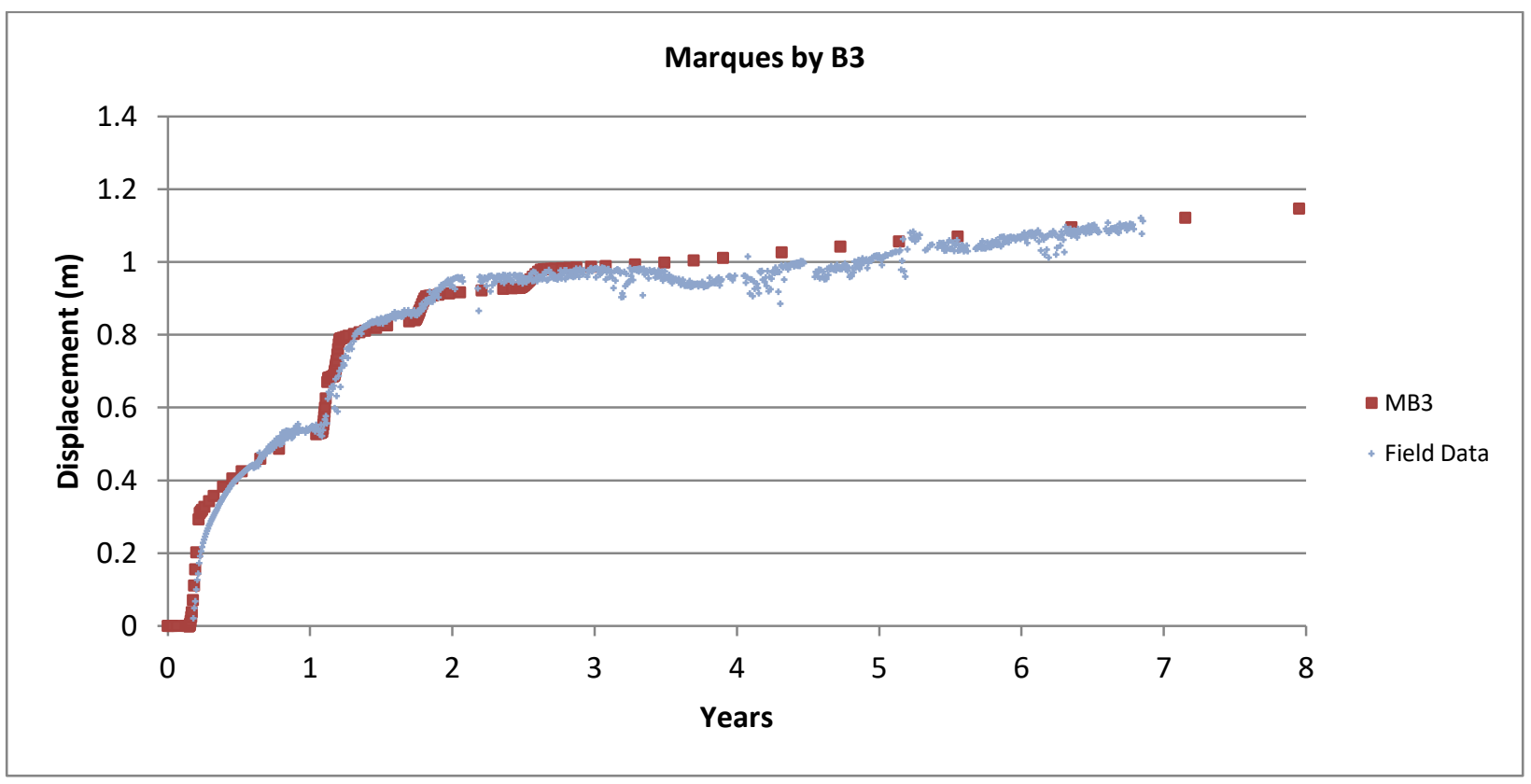

Figure 27: Marques-based model optimized to B3, displacement of B3 results 


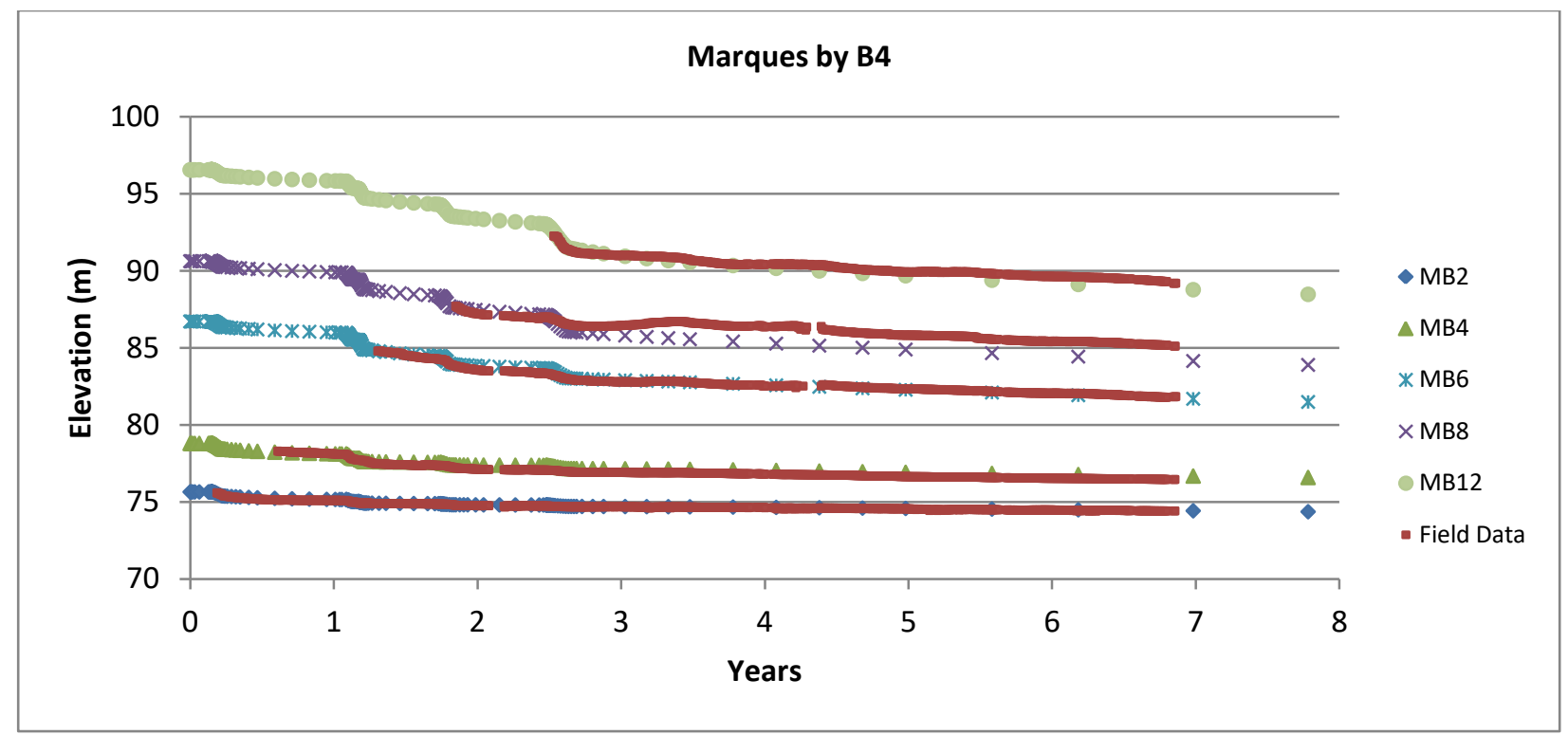

Figure 28: Marques-based model optimized to B4, elevation of even numbered bundles results

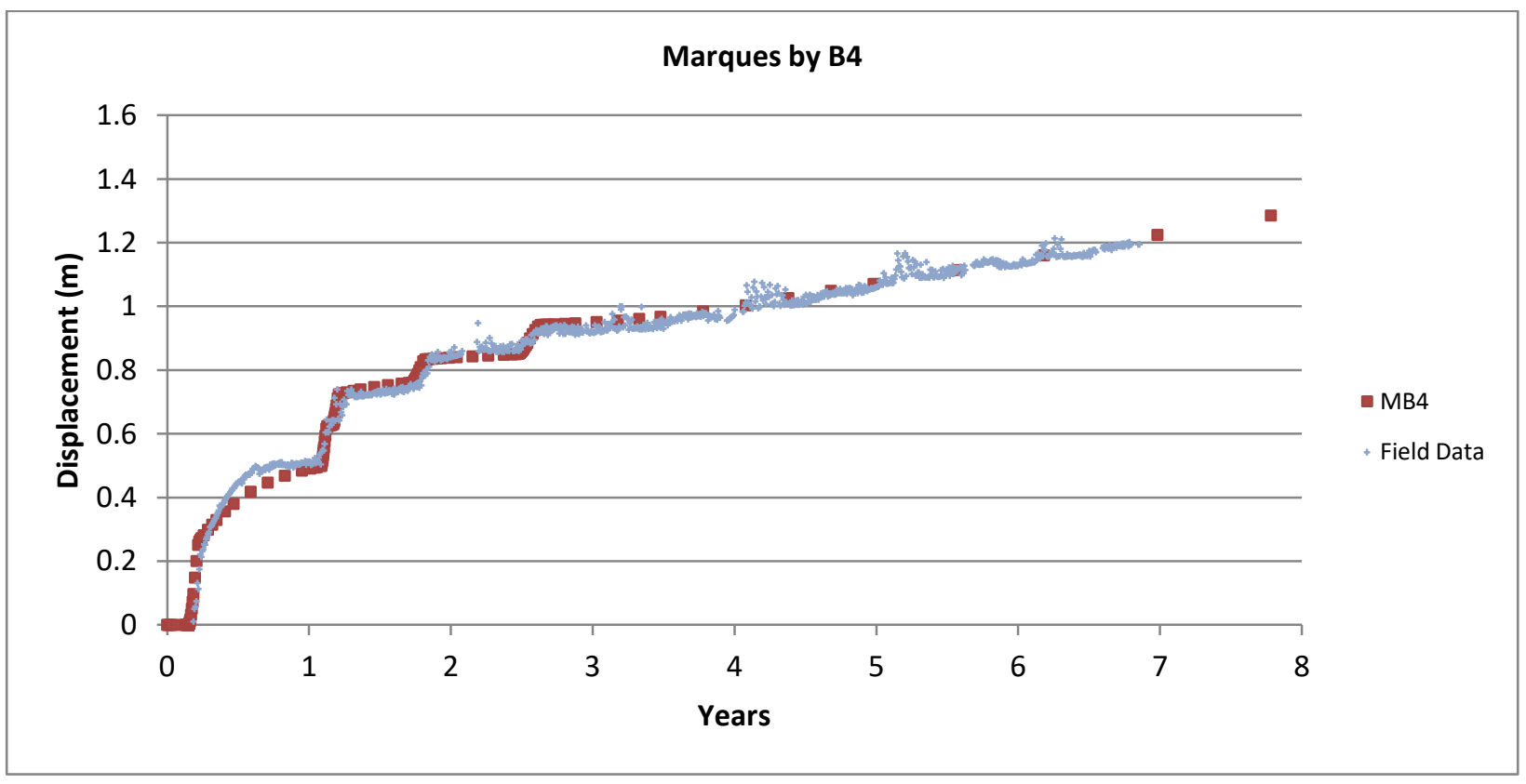

Figure 29: Marques-based model optimized to B4, displacement of B4 results 


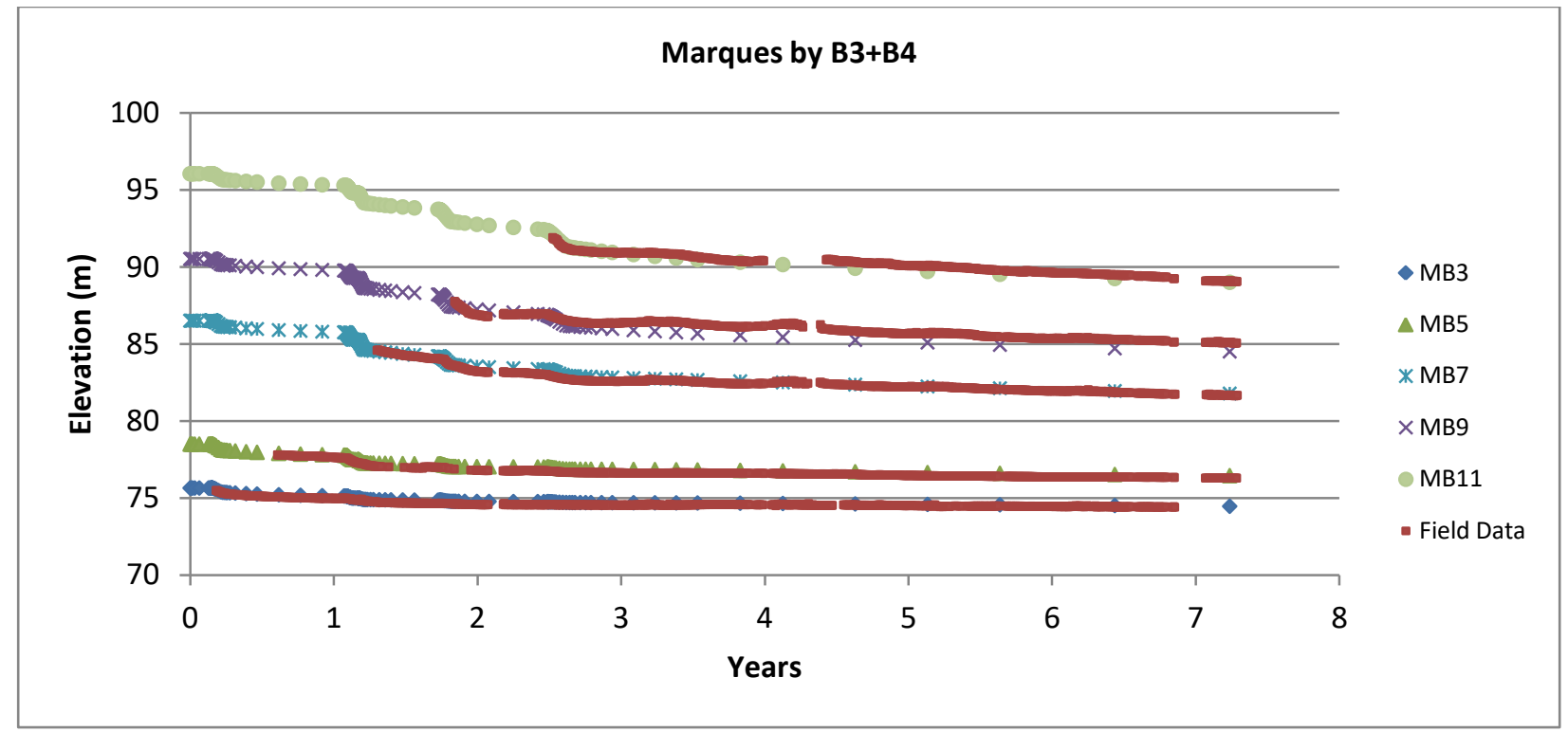

Figure 30: Marques-based model optimized to B3 and B4, elevation of odd numbered bundles results

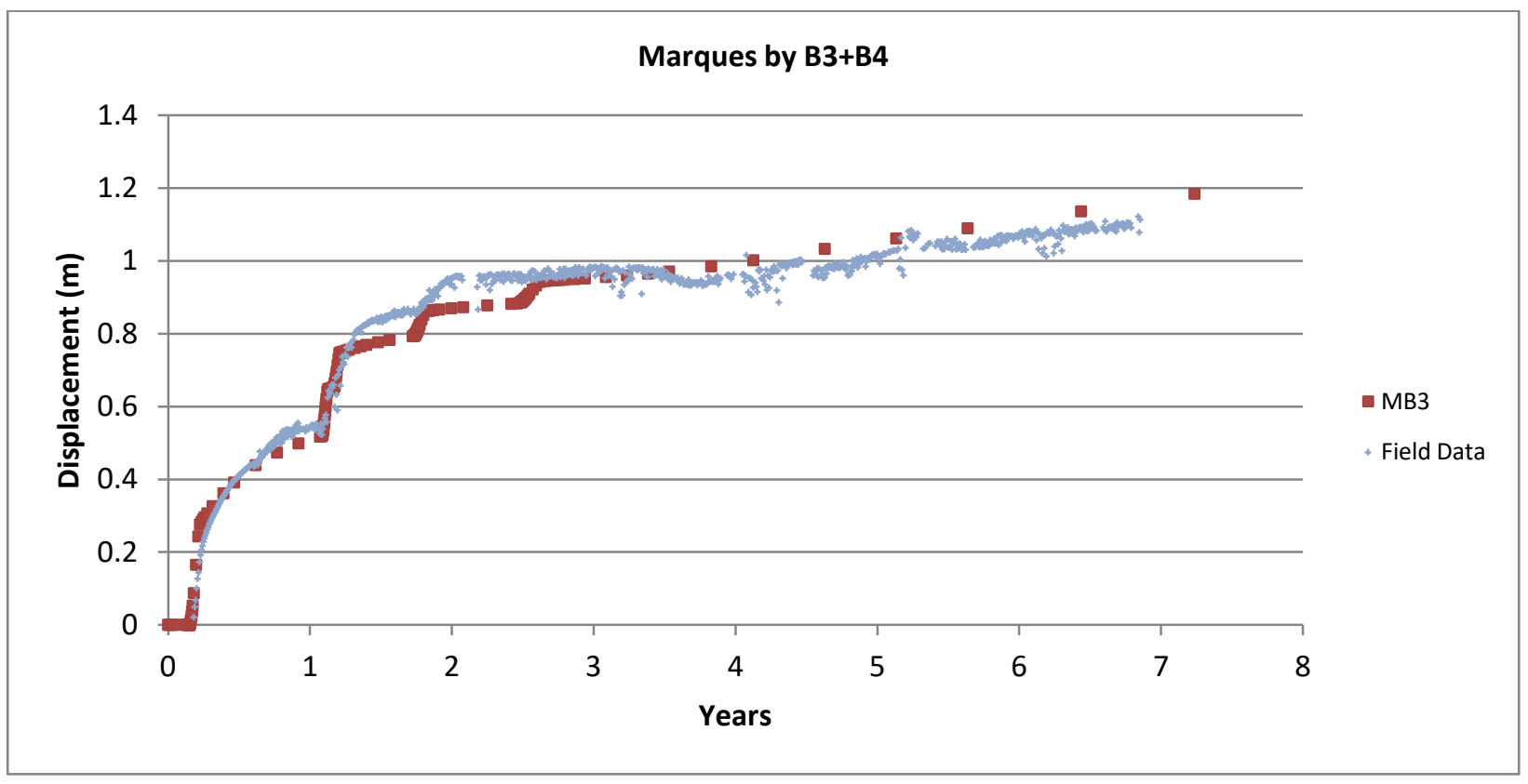

Figure 31: Marques-based model optimized to B3 and B4, displacement of B3 results 


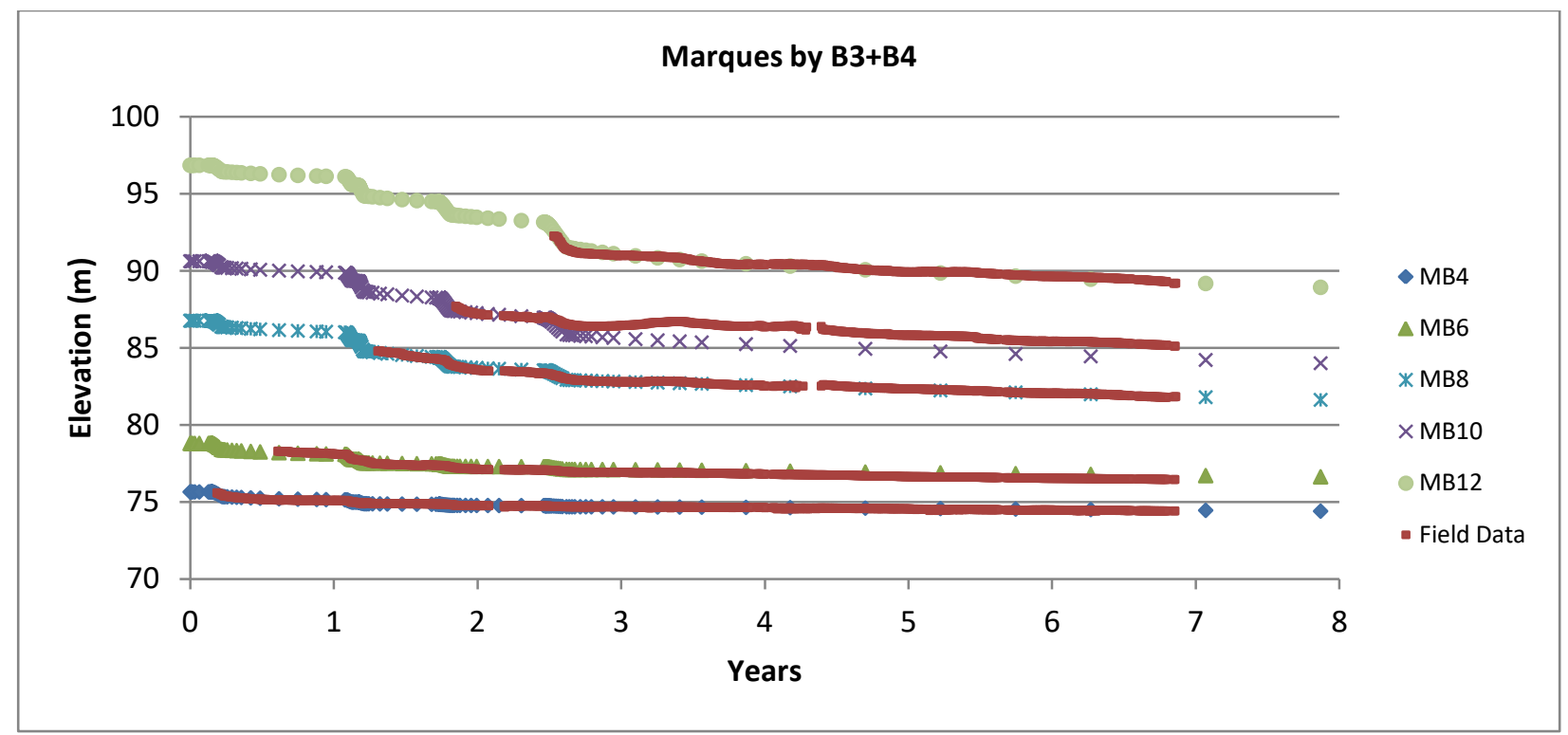

Figure 32: Marques-based model optimized to B3 and B4, elevation of even numbered bundles results

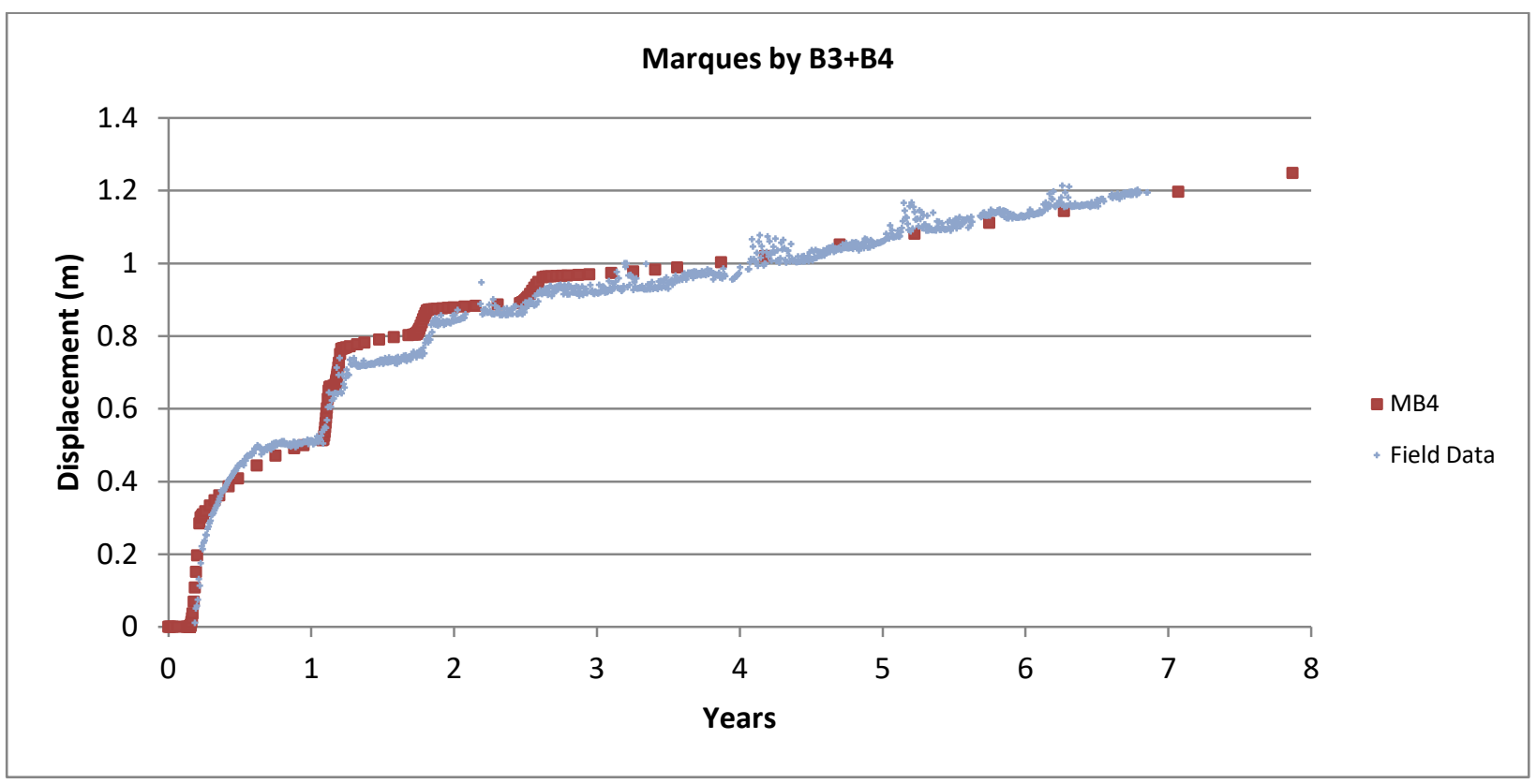

Figure 33: Marques-based model optimized to B3 and B4, displacement of B4 results 
Table 3: Optimization Parameters for the Marques-based model

\begin{tabular}{|l|l|l|l|l|l|}
\hline & B4 Optimization & B3 Optimization & B3 relative to B4 & $\begin{array}{l}\text { B3 + B4 } \\
\text { Optimization }\end{array}$ & $\begin{array}{l}\text { B3+B4 } \\
\text { relative to } \\
\text { B4 }\end{array}$ \\
\hline $\begin{array}{l}\mathrm{E}_{\mathrm{dg}} \text { [maximum } \\
\text { biodegradation } \\
\text { strain] }\end{array}$ & 0.26878 & 0.1589 & $\begin{array}{l}100 *(1- \\
0.1589 / 0.26878) \\
-41 \%\end{array}$ & 0.23307 & $13 \%$ \\
\hline $\begin{array}{l}\mathrm{E}_{\text {Start }} \text { [Starting } \\
\text { Young's Modulus] }\end{array}$ & $4.4306 \mathrm{E}[\mathrm{Pa}]$ & $4.0459 \mathrm{E} 5[\mathrm{~Pa}]$ & $9 \%$ & $4.0296 \mathrm{E} 5[\mathrm{~Pa}]$ & $9 \%$ \\
\hline $\begin{array}{l}\text { M [Young's } \\
\text { Modulus fitting } \\
\text { parameter] }\end{array}$ & 1.3842 & 0.48987 & $65 \%$ & 1.3617 & $2 \%$ \\
\hline $\begin{array}{l}\boldsymbol{N} \text { [Young's } \\
\text { Modulus fitting } \\
\text { parameter] }\end{array}$ & 0.63402 & 1.3758 & $-117 \%$ & 0.61614 & $3 \%$ \\
\hline $\begin{array}{l}\text { A [Poisson's ratio } \\
\text { fitting parameter] }\end{array}$ & 0.89687 & 0.97837 & $-9 \%$ & 0.92974 & $-4 \%$ \\
\hline $\begin{array}{l}\text { B [Poisson's ratio } \\
\text { fitting parameter] }\end{array}$ & 0.95396 & 1.00 & $-5 \%$ & 0.95758 & $-0.4 \%$ \\
\hline $\begin{array}{l}\boldsymbol{b} \text { [coefficient of } \\
\text { mechanical creep] }\end{array}$ & $3.7843 \mathrm{E}-6[1 / \mathrm{Pa}]$ & $3.6145 \mathrm{E}-6[1 / \mathrm{Pa}]$ & $4 \%$ & $3.7692 \mathrm{E}-6[1 / \mathrm{Pa}]$ & $0.4 \%$ \\
\hline $\begin{array}{l}\boldsymbol{c} \text { [rate constant } \\
\text { for mechanical } \\
\text { creep] }\end{array}$ & $7.7158 \mathrm{E}-8[1 / \mathrm{Pa}]$ & $5.9258 \mathrm{E}-8[1 / \mathrm{s}]$ & $23 \%$ & $6.7216 \mathrm{E}-8[1 / \mathrm{s}]$ & $13 \%$ \\
\hline RESULTING FIT & 0.30596 & 0.50637 & & 1.3905 & \\
\hline
\end{tabular}

The Marques-based model performed well, with good data matching in the construction phase and post-construction lifespan of the landfill, especially with the B4 optimization. Overall, each optimization of the Marques-based model can match the long-term magnitude of the field data very well, but is less accurate with the gradient of settlement behaviour. This different gradient for time-dependent settlement is most noticeable after the application of a waste lift (Figures 27, 29, 315, 33). While each optimization of the Marques-based model was adequate, there were some notable strengths and weaknesses between them. The B3+B4 simultaneous optimization, for the majority of parameters, sided much more closely to the results of the B4 optimization, with a maximum relative difference of $13 \%$ between their parameters. One possible reason for the B4 bias may have to do with the field data results for B3 at the application of the fifth waste lift (circa year 2.5). As seen in the B3 displacement plots above (Figures 27, 31), the B3 field data does not yield a profound instantaneous response during the placement of lift 5. This may be due to Bundle 3's presence in the side slope and resulting distance 
from the placement of lift 5, or perhaps due to a stiffening phenomenon from the hydraulic behaviour not yet accounted for in the FEM model. Regardless of the phenomenon, the FEM model does not account for an absence of loading or additional material stiffening, and produces an instantaneous response that is unseen in the field data, so it is likely that the optimization solver would more closely match the B4 data set where this response to loading was recorded.

The instantaneous response to loading was a notable improvement from the preceding iteration of the model introduced in McDonald et al. (2017). The inclusion of stress/strain dependent elastic material parameters have allowed for an instantaneous response that stiffens and decreases over time and is much less linear overall, as seen in Figures 27, 29, 31, and 33. This pseudo-nonlinearity is very apparent and effective between the application of the first and second lifts. The B4 and B3+B4 parameters for instantaneous behaviour $\left(E_{\text {Start }}, M, N, A, B\right)$ were all within expectations concerning published values in waste literature. The Young's moduli of $\sim 402 \mathrm{kPa}$ is consistent with the average values observed in literature :50 kPa to $800 \mathrm{kPa}$ (Sing and Flemming, 2008; Beaven and Powrie, 1995). $A$ and $B$ are consistent with the results of Ramaiah and Ramana (2017), and $M$ and $N$ were consistent with Janbu (1963)'s data .

The B3 optimization parameters were generally consistent with the time-dependent parameter estimates from other optimizations (B4 and $B 3+B 4)$, however, the instantaneous elastic response parameters featured a large inconsistency with the Young's modulus fitting parameters, $M$ and $N$; at a relative difference of $65 \%$ and $-117 \%$. A $N$ value of approximately 0.5 was expected to produce a more familiar trend of an increasing Young's modulus with stress - with a sharper initial increase, tapering off gradually with more stress applied. This behaviour is consistent with the values of 0.6 obtained for B4 and B3+B4. However, the $N$ value obtained for B3 was 1.3758. This larger value of $N$ would suggest a sharper increase in stiffness later in the simulation. The likely reason for this alternate behaviour is the 
aforementioned lack of instantaneous response to lift 5 seen within the B3 displacement data. Allowing a sharper increase in stiffness around the placement of lift 5 would allow a diminished response to loading and better matching to field data. This trend also seemed to influence the values of $A$ and $B$, which also control the instantaneous elastic response, and show a larger relative to difference of $-9 \%$ and $-5 \%$ respectively, compared to $\mathrm{B} 3+\mathrm{B} 4$ 's $-4 \%$ and $-0.4 \%$. If the control parameter, $N$, is constrained during optimization to remain below a value of 1 , a good fit can still be achieved, but not a better fit than without the constraint. The 'constrained' parameters more closely align with the values obtained for B4 and B3+B4. Observing the relative difference of the 'constrained' B3 parameters, the correspondence to B4 is improved from the 'unconstrained' values across all the parameters, except for the coefficient of mechanical creep, $b$, which shows a deviation from $5 \%$ to $-7 \%$. These constrained parameters are presented in the Table 4.

Table 4: B3 Optimization values with $\mathbf{N}$ value constrained to expected trends for Marques-based model

\begin{tabular}{|l|l|l|l|l|l|}
\hline & B4 Optimization & $\begin{array}{l}\text { Constrained B3 } \\
\text { Optimization }\end{array}$ & $\begin{array}{l}\text { B3 relative } \\
\text { to B4 }\end{array}$ & $\begin{array}{l}\text { B3 + B4 } \\
\text { Optimization }\end{array}$ & $\begin{array}{l}\text { B3+B4 relative } \\
\text { to B4 }\end{array}$ \\
\hline $\begin{array}{l}\mathrm{E}_{\mathrm{dg}} \text { [maximum } \\
\text { biodegradation } \\
\text { strain] }\end{array}$ & 0.26878 & 0.16054 & $40 \%$ & 0.23307 & $13 \%$ \\
\hline $\begin{array}{l}\mathrm{E}_{\text {Start }} \text { [Starting } \\
\text { Young's Modulus] }\end{array}$ & $4.4306 \mathrm{E}[\mathrm{Pa}]$ & $3.9463 \mathrm{E}[\mathrm{Pa}]$ & $11 \%$ & $4.0296 \mathrm{E} 5[\mathrm{~Pa}]$ & $9 \%$ \\
\hline $\begin{array}{l}\text { M [Young's Modulus } \\
\text { fitting parameter] }\end{array}$ & 1.3842 & 1.3604 & $2 \%$ & 1.3617 & $2 \%$ \\
\hline $\begin{array}{l}\boldsymbol{N} \text { [Young's Modulus } \\
\text { fitting parameter] }\end{array}$ & 0.63402 & 0.54695 & $14 \%$ & 0.61614 & $3 \%$ \\
\hline $\begin{array}{l}\boldsymbol{A} \text { [Poisson's ratio } \\
\text { fitting parameter] }\end{array}$ & 0.89687 & 0.90988 & $-1 \%$ & 0.92974 & $-4 \%$ \\
\hline $\begin{array}{l}\text { B [Poisson's ratio } \\
\text { fitting parameter] }\end{array}$ & 0.95396 & 0.92683 & $3 \%$ & 0.95758 & $-0.4 \%$ \\
\hline $\begin{array}{l}\boldsymbol{b} \text { [coefficient of } \\
\text { mechanical creep] }\end{array}$ & $3.7843 \mathrm{E}-6[1 / \mathrm{Pa}]$ & $4.0283 \mathrm{E}-6[1 / \mathrm{Pa}]$ & $-7 \%$ & $3.7692 \mathrm{E}-6[1 / \mathrm{Pa}]$ & $0.4 \%$ \\
\hline $\begin{array}{l}\boldsymbol{c} \text { [rate constant for } \\
\text { mechanical creep] }\end{array}$ & $7.7158 \mathrm{E}-8[1 / \mathrm{Pa}]$ & $6.0146 \mathrm{E}-8[1 / \mathrm{s}]$ & $22 \%$ & $6.7216 \mathrm{E}-8[1 / \mathrm{s}]$ & $13 \%$ \\
\hline RESULTING FIT & 0.30596 & 0.52316 & & 1.3905 & \\
\hline
\end{tabular}

Regarding the long-term behaviour, the B3 optimization simulated significantly less long-term settlement in the post-construction lifespan (3 years+) for both mechanical creep and biodegradation- 
induced settlement when compared to B4. As a result, the B3 optimization underestimated the longterm settlement of the upper bundles, with an increasing error of prediction with increasing height of the bundles (Figure 26). Conversely, the B4 optimization overestimated much of the long-term behaviour with its higher creep and biodegradation parameters (Figure 28). The B3+B4 optimization produced creep and biodegradation parameters that were roughly an average of the individual B3 and B4 parameters, but weighed more closely to B4, with a relative difference of $9 \%$. This average set of parameters provided the best estimate at predicting the upper bundles, with good accuracy achieved on both the odd and even numbered bundle sets (Figure 30, 32). However, this set of average parameters did not yield great data matching of the lower bundles (Figures 31, 33), but the match is fair at the least.

Regarding the parameter controlling the magnitude of biodegradation-induced settlement, $E_{\mathrm{DG}}$, all three optimizations, in addition to the 'constrained' optimization, yielded values fairly consistent with literature, lying on the higher side of published values. It should be noted that the ranges for $E_{\mathrm{DG}}$ found in literature are quite wide, due to the variability of waste tested across landfills of varying age, climate, waste composition, and the effort to separate mechanical and biodegradation behaviours. Typical values of $E_{\mathrm{DG}}$ range from 0.11 - 0.24 (Marques et al., 2003; Babu et al., 2010; Van Geel and Murray, 2015).

Van Geel and Murray (2015) applied the Marques et al. (2003) numerical model to the same landfill presented in this thesis. The Marques-based model presented within this thesis is a modified version of the original Marques et al. (2003) model and does not use all the same parameters as Van Geel and Murray (2015). Compared to the original Marques et al. (2003) model (seen in Section 2, Equation 2-H), only the mechanical creep term remains entirely the same in the Marques-based model presented in this thesis. The Marques-based model from this thesis does feature a different biodegradation term based on Berquist (2017)'s thermal model, but the parameter $E_{D G}$ is featured in both models and 
functions the same in both. Van Geel and Murray (2015)'s odd-even bundle results are compared to the B3 and B4 individual optimizations in Table 5. A plot of Van Geel and Murray (2015)'s best fit model using the parameters in Table 5 is shown in Figure 34.

Table 5: Comparison to Van Geel and Murray (2015) Parameters

\begin{tabular}{|l|l|l|l|l|}
\hline Parameter & $\begin{array}{l}\text { This Thesis } \\
\text { B3 Results }\end{array}$ & $\begin{array}{l}\text { Van Geel and } \\
\text { Murray (2015) } \\
\text { Odd bundles }\end{array}$ & $\begin{array}{l}\text { This Thesis } \\
\text { B4 results }\end{array}$ & $\begin{array}{l}\text { Van Geel and } \\
\text { Murray (2015) } \\
\text { Even bundles }\end{array}$ \\
\hline$b[1 / \mathrm{Pa}]$ & $3.6145 \mathrm{E}-6$ & $1.19 \mathrm{E}-6$ & $3.7843 \mathrm{E}-6$ & $8.32 \mathrm{E}-7$ \\
\hline$c[1 / \mathrm{s}]$ & $5.9258 \mathrm{E}-8$ & $2.407 \mathrm{E}-7$ & $7.7158 \mathrm{E}-8$ & $2.4769 \mathrm{E}-7$ \\
\hline $\mathrm{E}_{\mathrm{DG}}$ & 0.1589 & 0.112 & 0.26878 & 0.234 \\
\hline
\end{tabular}

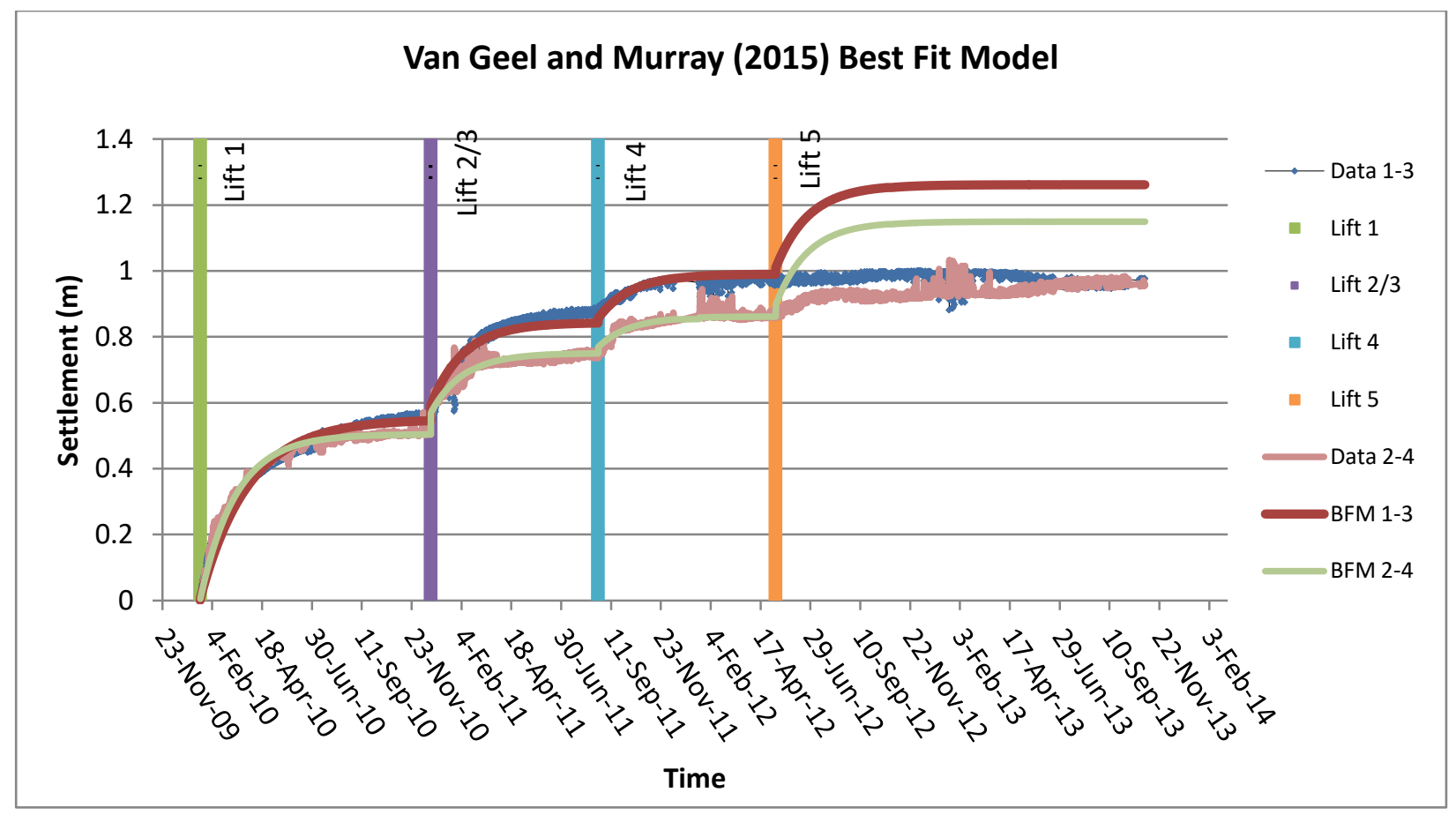

Figure 34: Van Geel and Murray (2015)'s measured and simulated settlement for layers between Bundles 1-3 and Bundles 2-4 (BFM = Best Fit Model)

Van Geel and Murray (2015)'s dataset and model setup varies slightly from the Marques version of the model seen in this thesis. Primarily, Van Geel and Murray (2015) had access to fewer years of data at the time, and could only fit their model to approximately 3.5-4 years of data points, versus the 7 years used in this thesis. However, Van Geel and Murray (2015) only fit their model up to just before the placement 
of the fifth waste lift due to unexpected trends and an overestimated response when fitting past the fifth waste lift. This means Van Geel and Murray (2015) were only able to fit to just over 2 years of field data. The divergence of the field data from the best fit model seen at the placement of lift 5 in Figure 34 is due to Van Geel and Murray (2015) only being able to match this first 2 years of field data.

Additionally, the lift thicknesses used in Van Geel and Murray (2015) and the Marques version of the model in this thesis are nearly the same to the exception of lift 5. Van Geel and Murray (2015) utilized a fifth waste lift that was approximately $2 \mathrm{~m}$ larger than the fifth waste lift used in this thesis. The lift size from this thesis is believed to be more representative what was actually placed in the field. This larger fifth waste lift contributes at least in part to Van Geel and Murray (2015)'s over estimating of behaviour past the placement of the fifth waste lift (Figure 34), but does not contribute to the fitting or estimation of the derived mechanical parameters. The larger thickness of the fifth waste lift used by Van Geel and Murray (2015) may be why the best fit model's post lift 5 response is able to better match the field data for upper bundle layers, as seen in Figure 35. As seen in Table 5, Van Geel and Murray (2015)'s results align with this thesis when comparing trends observed in the odd bundle data sets to trends seen in the even bundle data sets. Both studies confirm lower $E_{D G}$ terms, higher creep coefficients, and lower creep rate constants in the odd numbered bundles when compared to the even numbered bundles. The range for maximum biodegradation-induced strain from B3 and B4, $0.1589-0.26878$, presented above is fairly consistent with Van Geel and Murray (2015), 0.112-0.234, and not too far off the original range published by Marques et al. (2003). Marques (2003) published a range of $E_{D G}$ at $0.131-0.214$ (avg. = $0.159)$.

The difference in magnitude between these values of $E_{\mathrm{DG}}$ can be explained by the method in which $\mathrm{E}_{\mathrm{DG}}$ was implemented in each model. Marques (2003) utilizes a user-defined starting time for biodegradation. Van Geel and Murray (2015) toggles biodegradation to occur only for lifts that reach a minimum sufficient temperature for biodegradation to occur. Within Van Geel and Murray's dataset, 
lifts 1,2 , and 3 did not reach a sufficient temperature, so biodegradation-induced settlement is only considered for lifts 4 and 5. Van Geel and Murray (2015) then obtained the rate constant for biodegradation $(d)$ and $E_{\mathrm{DG}}$ by regressing parameters from the settlement data from layers between Bundles 9-11 and Bundles 10-12 (which includes material from both the fourth and fifth waste lifts). This regression was done using mechanical parameters obtained from fit on the layers between Bundles 1-3 and Bundles 2-4. The Marques-based model presented in this thesis uses an energy curve approximation based on a simulation of the thermal data, as shown in Figures 22 and 23. Using the energy curves provides a more accurate alternative to the rate constant (d) from Marques et al. (2003) as these parameters vary with temperature. The value of $E_{\mathrm{DG}}$ for the Marques-based model was derived from the settlement data of Bundle 3 and Bundle 4, both of which feature biodegradation less prominently than the upper bundles. Van Geel and Murray (2015) may have a less accurate approach for determining the rate of biodegradation, but were able to optimize $E_{\mathrm{DG}}$ on bundles with more prominent biodegradation behaviour.

Van Geel and Murray (2015)'s mechanical creep coefficients $(b)$ are much smaller, while their creep rate constant $(c)$ is much larger compared to both the B3 and B4 results. These differences are primarily the results of the shorter dataset used by Van Geel and Murray (2015) versus the larger dataset used in this thesis. Because of the short dataset available to Van Geel and Murray (2015), the regressed parameters are unable to fully capture the long-term behaviour simply because there is not enough long-term behaviour emergent from 2 years of data. This issue is apparent when analyzing Van Geel and Murray (2015)'s prediction of upper waste layers using their best fit parameters, as seen Figure 35. 


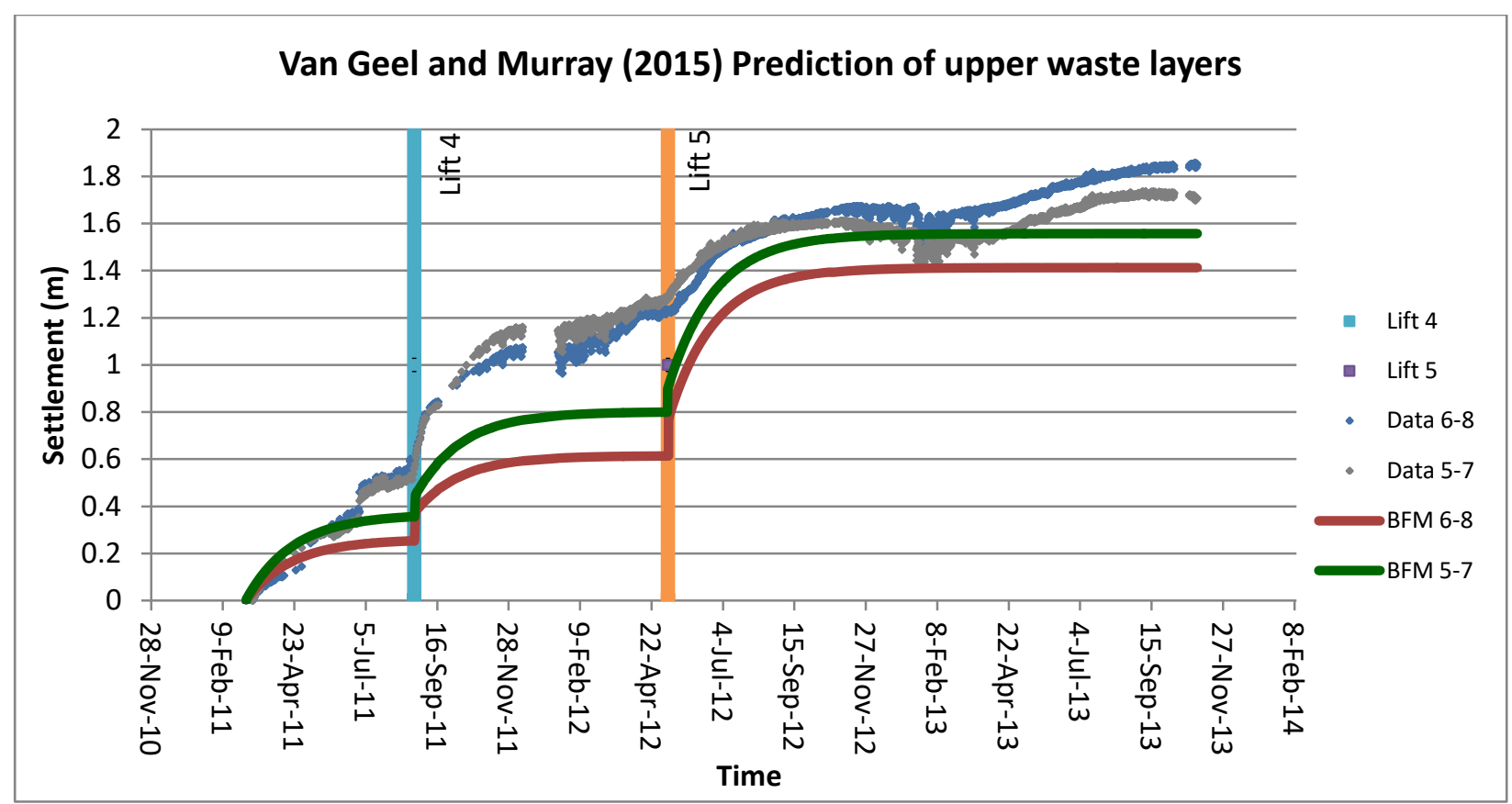

Figure 35: Van Geel and Murray (2015)'s prediction of layers between Bundles 6-8 and Bundles 5-7 using best fit parameters Figure 35 shows Van Geel and Murray (2015)'s best fit model's settlement predictions in comparison to the field data. The data matching for the bundle layers 5-7 and 6-8 are noticeably not as good as the bundle predictions from the Marques-based model seen in Figures 26 and 28 for Bundles 5, 6, 7, and 8. Van Geel and Murray (2015)'s prediction of waste in layers between Bundles 6-8 and Bundles 5-7 (which feature no biodegradation term) shows a plateau of settlement behaviour in between the placement of each lift, while the field data maintains an ongoing time-dependent settlement for each placement of waste. This plateau in indicative that the mechanical creep behaviour has finished quickly at a lesser magnitude than the field data. This is due to Van Geel and Murray (2015)'s smaller creep coefficient and larger creep constant than the B3 and B4 optimizations. A smaller creep constant yields less settlement due to mechanical creep. A larger creep rate constant, which controls the gradient, yields a completed mechanical creep effect in a shorter period of time. 


\subsection{Viscoelastic Model Results}

The viscoelastic model also performed very well. In regards to matching the field data, it provided the best data matching to the odd bundles. Additionally, it requires one less parameter than the Marquesbased model. Less required parameters can aid in making the viscoelastic model adaptable to other landfill sites. Additionally, having fewer parameters enables a quicker optimization process. The optimized parameters and resulting fit can be seen in Table 6 and Figures 36 - 43. Figures 36-43 show the plots of the simulated and field settlement based on optimizations using Bundle 3, Bundle 4, and Bundles 3 and 4 together. Figures 36,38, 40, and 42 show the elevation of all bundles. Figures 37, 39, 41, and 43 show the corresponding simulated and field displacement at the bundles used for optimization.

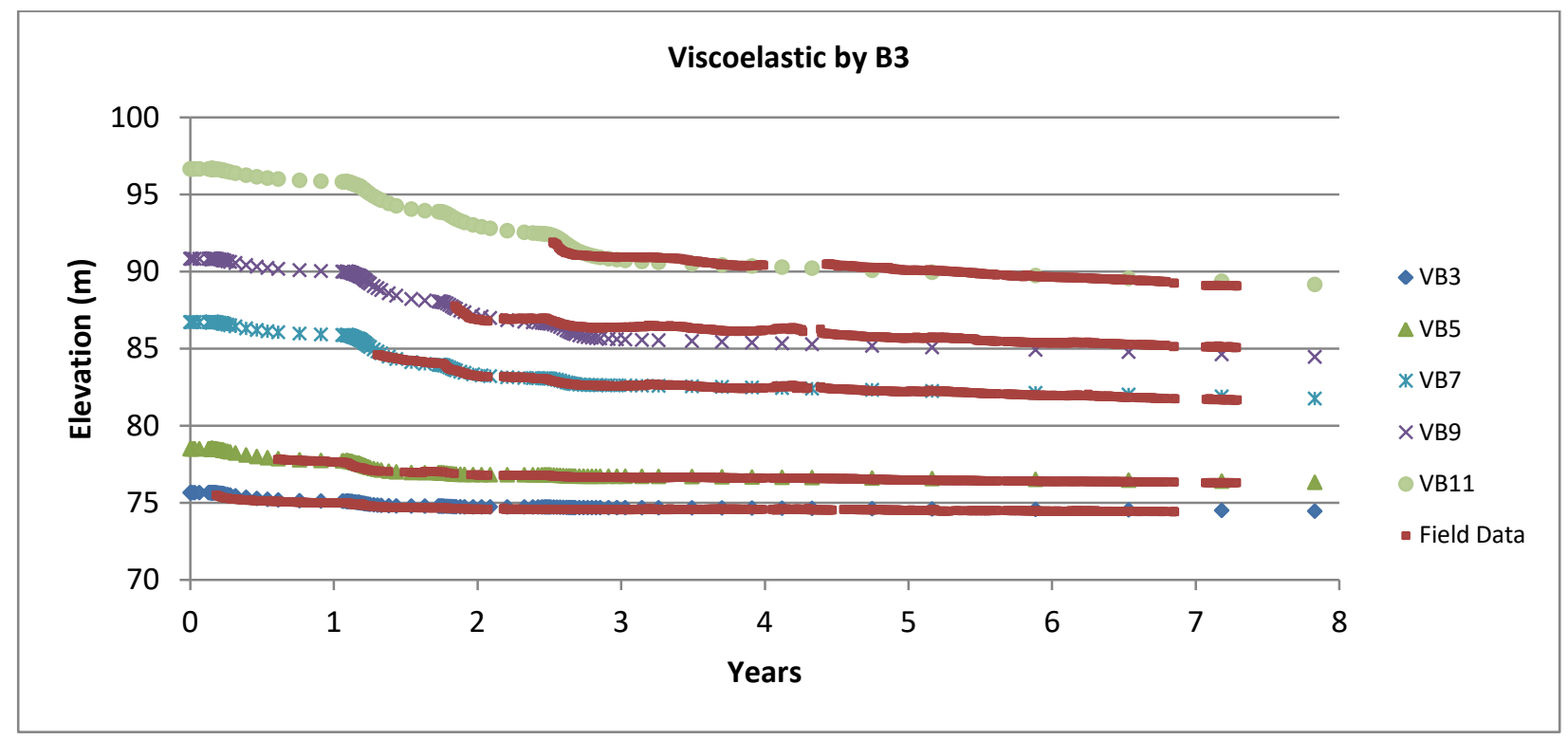

Figure 36: Viscoelastic model optimized to B3, elevation of odd numbered bundles results 


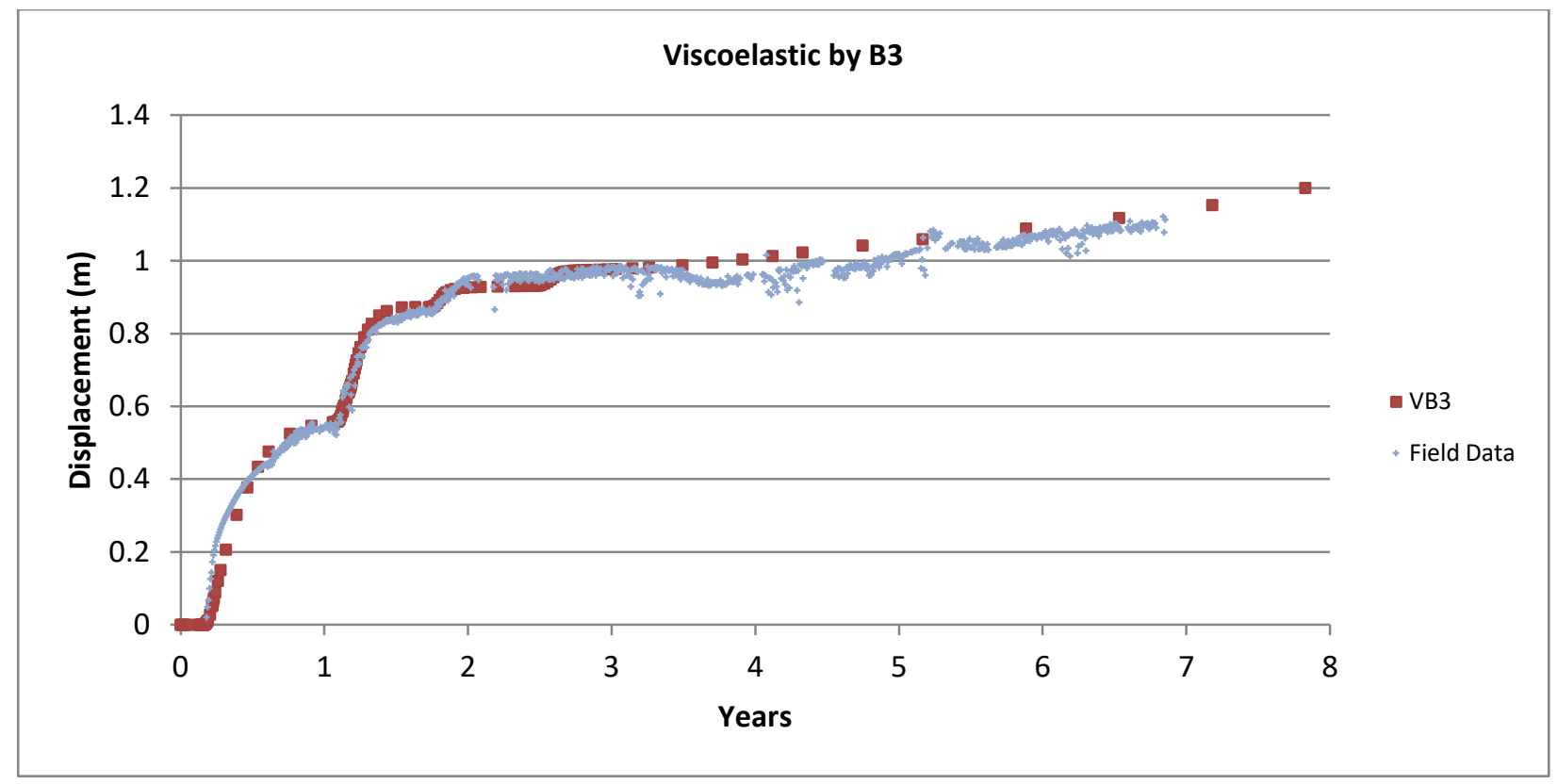

Figure 37: Viscoelastic model optimized to B3, displacement of B3 results

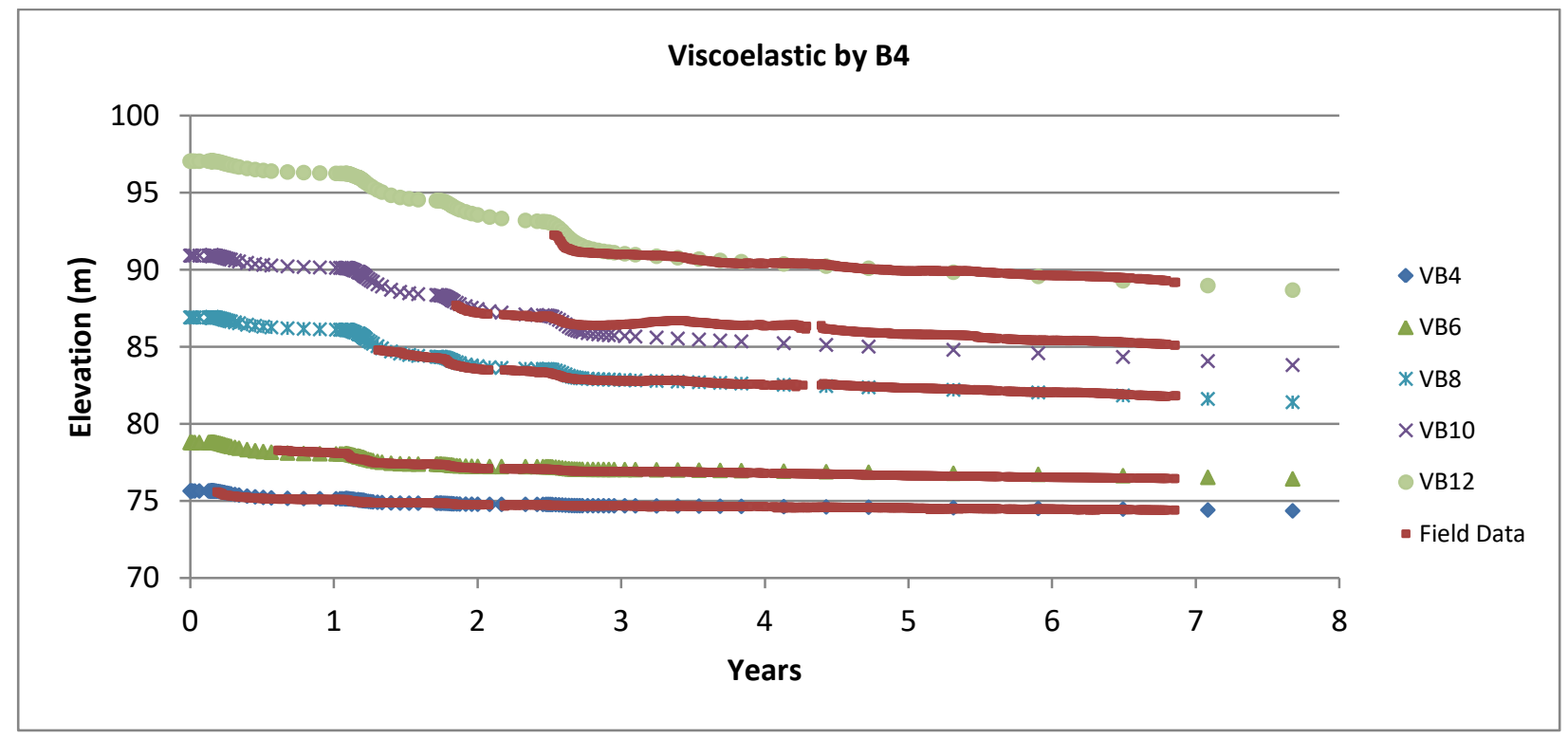

Figure 38: Viscoelastic model optimized to B4, elevation of even numbered bundles results 


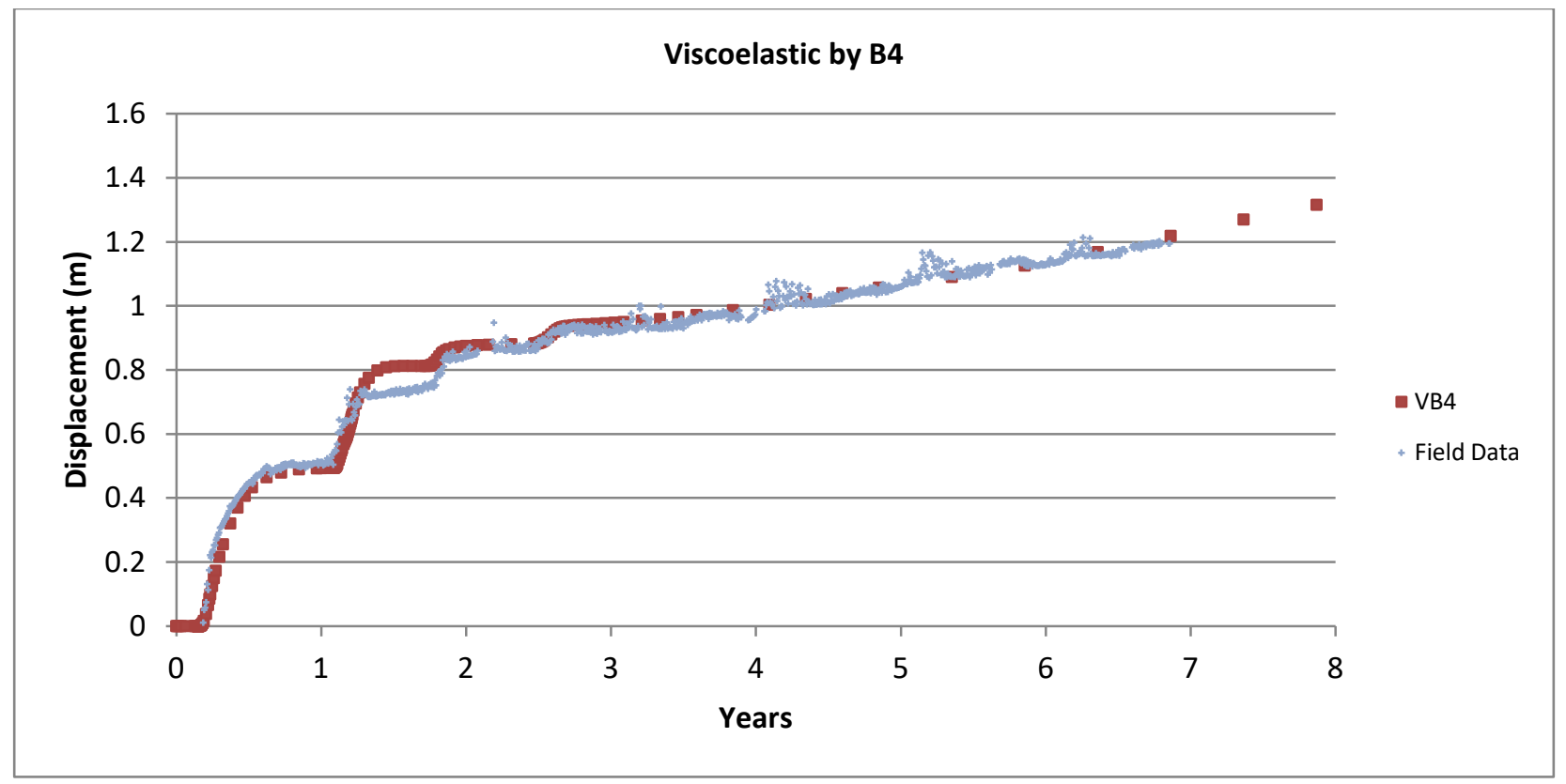

Figure 39: Viscoelastic model optimized to B4, displacement of B4 results

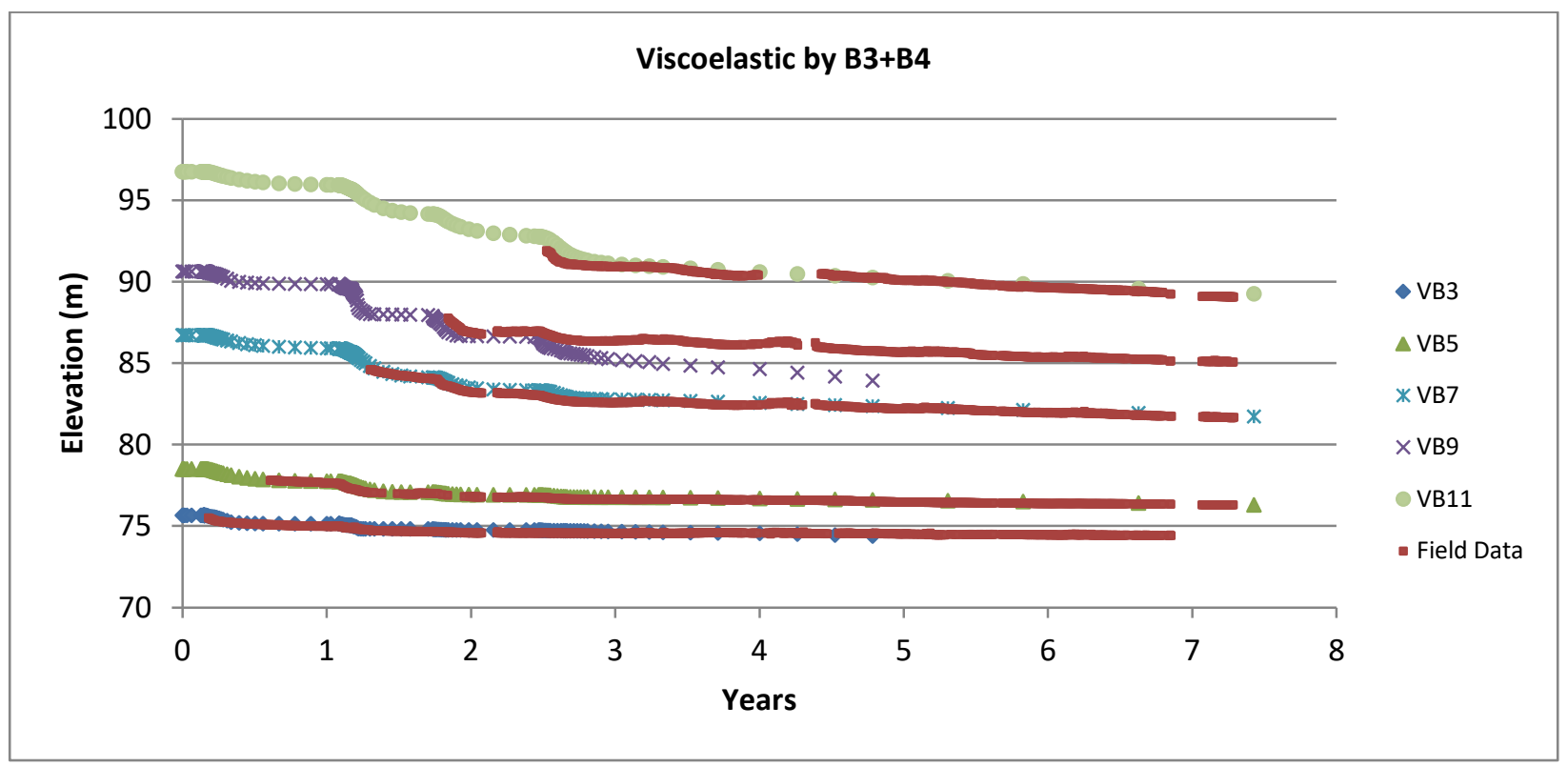

Figure 40: Viscoelastic model optimized to B3 and B4, elevation of odd numbered bundles results 


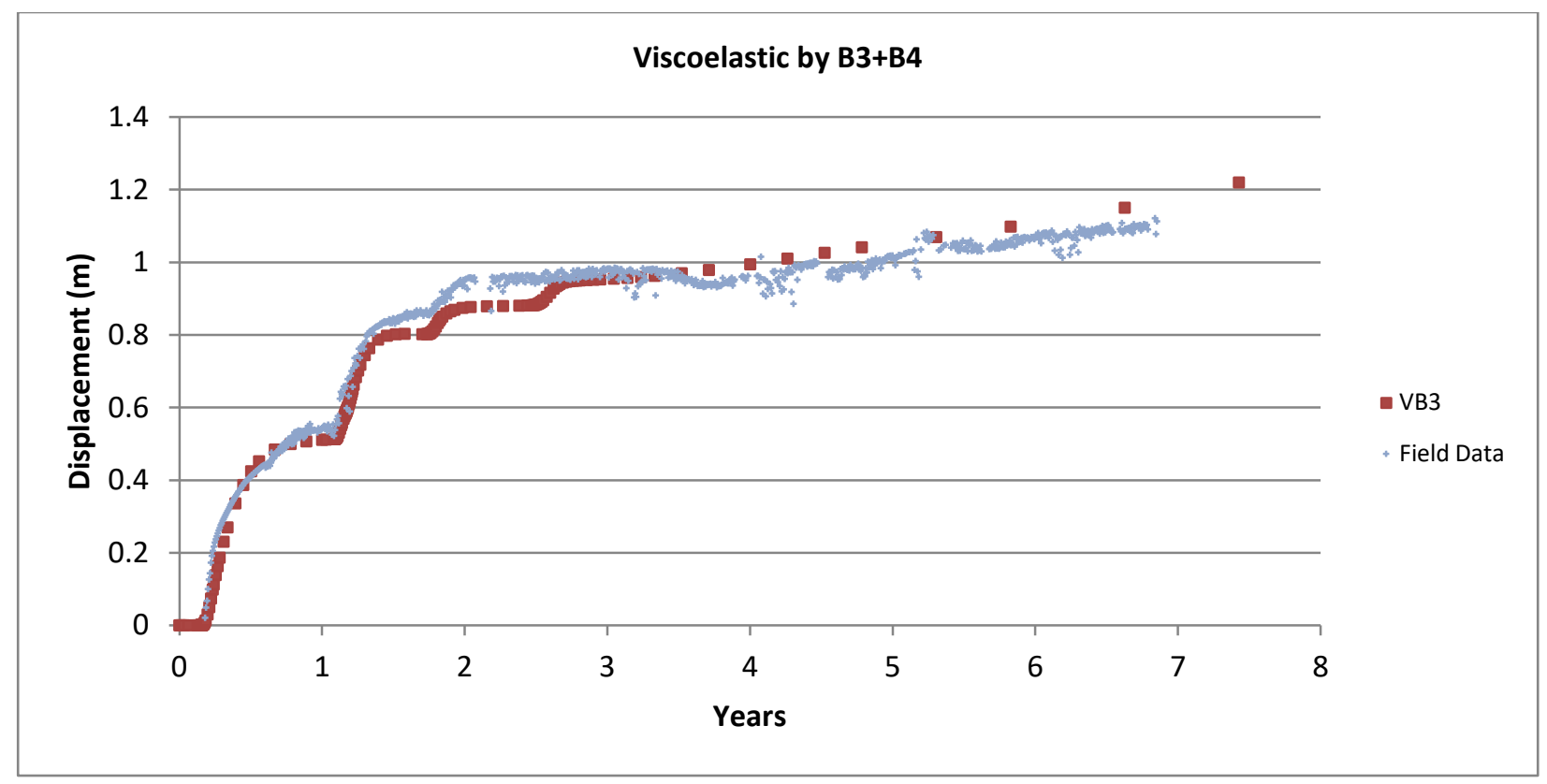

Figure 41: Viscoelastic model optimized to $B 3$ and B4, displacement of B3 results

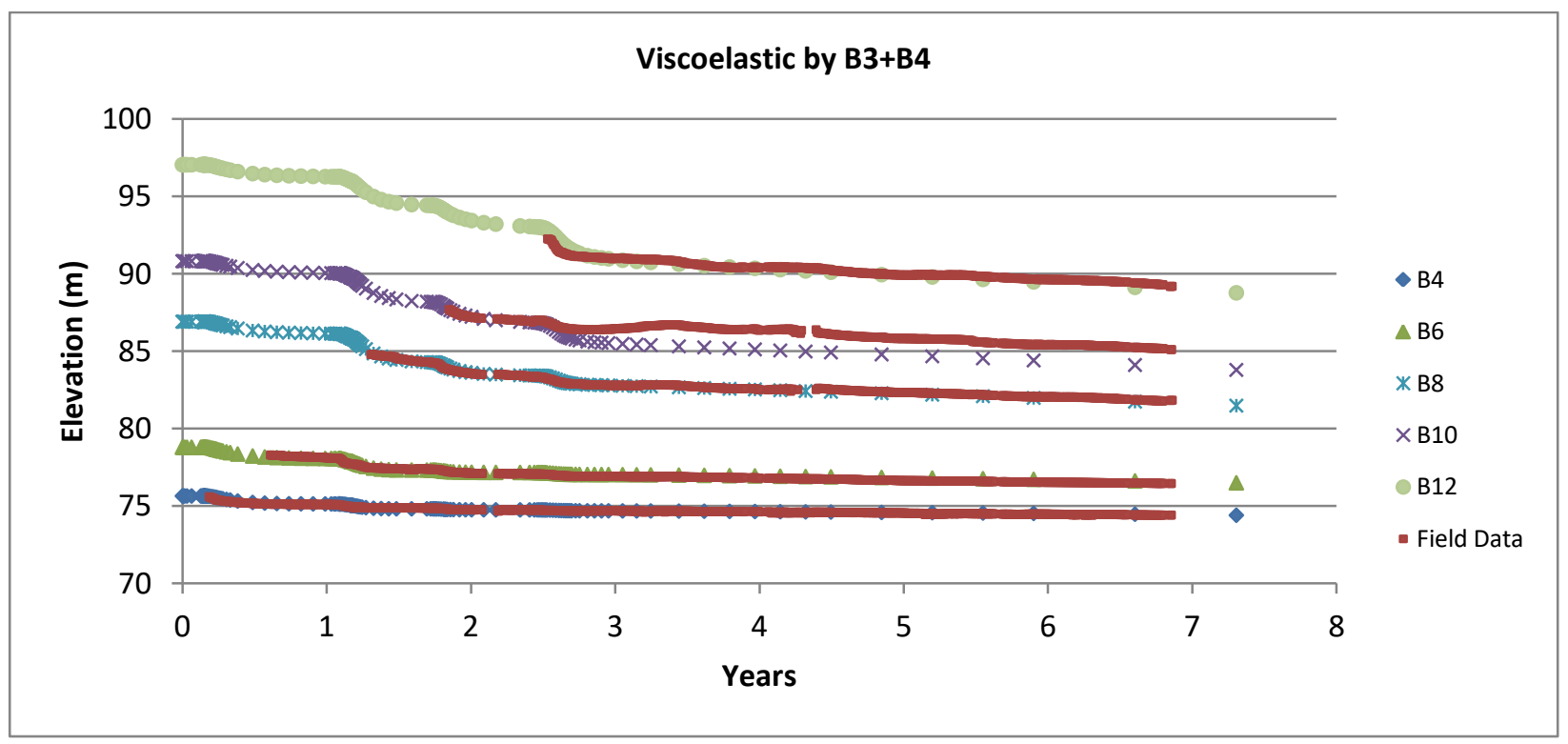

Figure 42: Viscoelastic model optimized to B3 and B4, elevation of even numbered bundles results 


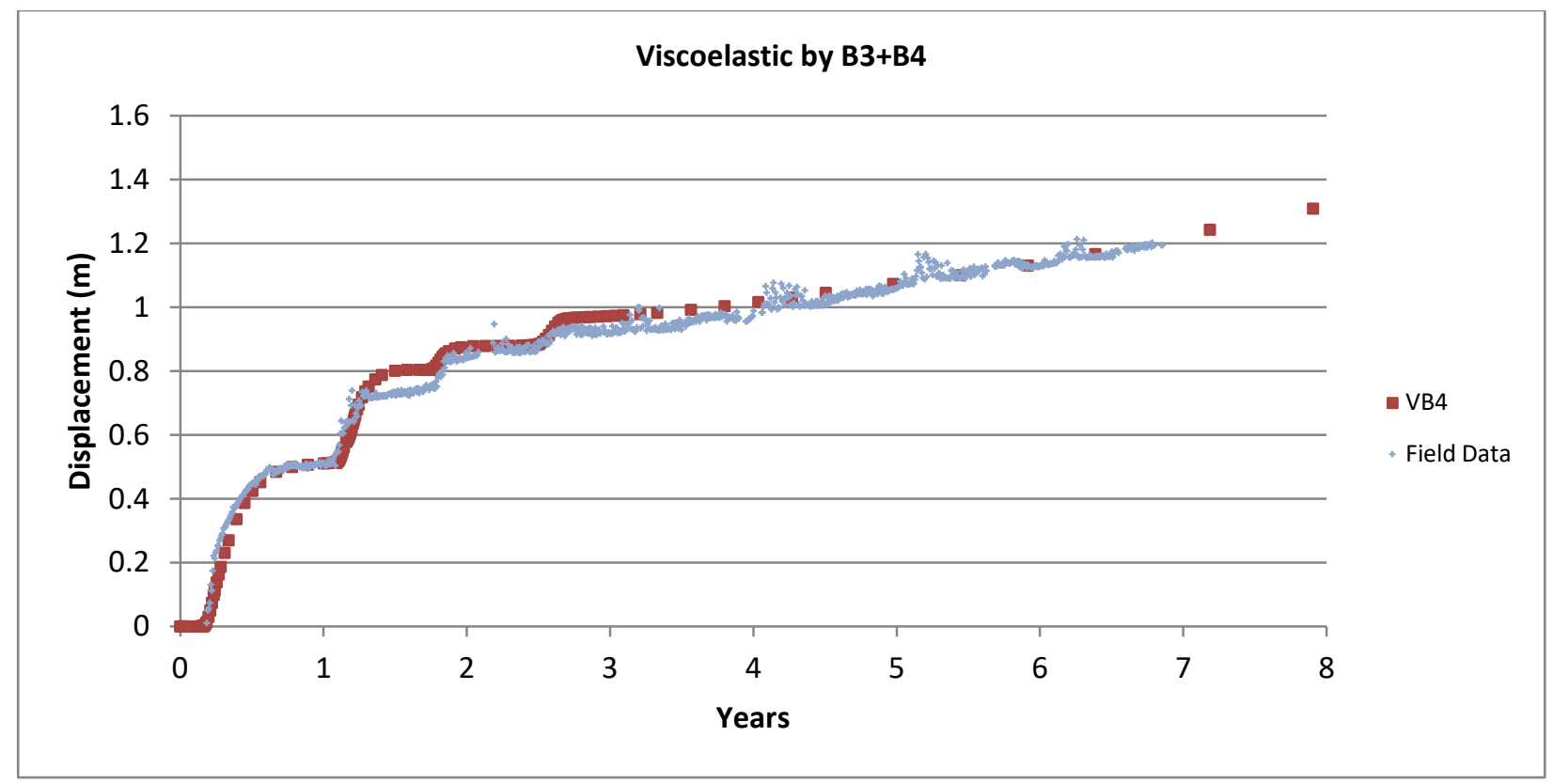

Figure 43: Viscoelastic model optimized to B3 and B4, displacement of B4 results

Table 6: Optimization Parameters for the viscoelastic model

\begin{tabular}{|l|l|l|l|l|l|}
\hline & B4 Optimization & B3 Optimization & $\begin{array}{l}\text { B3 relative } \\
\text { to B4 }\end{array}$ & $\begin{array}{l}\text { B3 + B4 } \\
\text { Optimization }\end{array}$ & $\begin{array}{l}\text { B3+B4 relative } \\
\text { to B4 }\end{array}$ \\
\hline $\begin{array}{l}\mathrm{E}_{\mathrm{dg}} \text { [maximum } \\
\text { biodegradation } \\
\text { strain] }\end{array}$ & 0.32755 & 0.3287 & $-0.4 \%$ & 0.32180 & $2 \%$ \\
\hline $\begin{array}{l}\mathrm{E}_{\text {Start }} \text { [Starting } \\
\text { Young's Modulus] }\end{array}$ & $1.3184 \mathrm{E}[\mathrm{Pa}]$ & $1.7138 \mathrm{E}[\mathrm{Pa}]$ & $-30 \%$ & $1.2771 \mathrm{E} 5[\mathrm{~Pa}]$ & $3 \%$ \\
\hline $\begin{array}{l}\text { M [Young's Modulus } \\
\text { fitting parameter] }\end{array}$ & 1.4761 & 0.42724 & $71 \%$ & 1.4221 & $4 \%$ \\
\hline $\begin{array}{l}\text { N [Young's Modulus } \\
\text { fitting parameter] }\end{array}$ & 0.66542 & 1.4217 & $-113 \%$ & 0.62977 & $5 \%$ \\
\hline $\begin{array}{l}\text { A [Poisson's ratio } \\
\text { fitting parameter] }\end{array}$ & 0.87698 & 1.0074 & $-15 \%$ & 0.88887 & $-1 \%$ \\
\hline $\begin{array}{l}\text { B [Poisson's ratio } \\
\text { fitting parameter] }\end{array}$ & 0.93234 & 1.000 & $-7 \%$ & 0.93642 & $-0.4 \%$ \\
\hline$\tau$ [Relaxation time] & $1.4375 \mathrm{E} 7[\mathrm{~s}]$ & $1.9820 \mathrm{E} 7[\mathrm{~s}]$ & $-38 \%$ & $1.4283 \mathrm{E} 7[\mathrm{~s}]$ & $0.6 \%$ \\
\hline RESULTING FIT & 0.41329 & 0.37624 & & 1.3844 & \\
\hline
\end{tabular}

The viscoelastic model yielded good predictions of both short-term and long-term settlement behaviour across each optimization. By observation of Figures 37, 39, 41, and 43, the viscoelastic model also offers a good gradient for settlement behaviour that allows a smooth transition between instantaneous 
behaviour and time-dependent, long-term behaviour. This gradient is comparable to the field data's behaviour and is reflective of realistic waste behaviour. Each of the viscoelastic model's optimizations yielded acceptable parameters. B3's resulting fit was best among the viscoelastic optimizations, but its parameters controlling the elastic material behaviour were questionable. The large relative difference between B3 and B4 parameters, with a maximum difference of $-113 \%$, suggested significantly different stiffening behaviour occurring across the two bundles. As was the case with the Marques-based model, the parameters $M$ and $N$ were subject to an unexpected pattern of stiffening behaviour during the B3 optimization run. To reiterate, this different stiffening behaviour is thought to be a compensation for Bundle 3's lack of instantaneous response to the placement of lift 5. Constraining $N$ to behave as expected provided parameters that were more consistent with B4 and B3+B4, but the resulting fit for the data was not as good. Despite the worsened fit, the 'constrained' B3 parameters are much more consistent with B4 and B3+B4. This constrained set of viscoelastic parameters are shown below in Table 7.

Table 7: B3 Optimization values with $N$ value constrained to expected trends for viscoelastic model

\begin{tabular}{|l|l|l|l|l|l|}
\hline & B4 Optimization & $\begin{array}{l}\text { Constrained B3 } \\
\text { Optimization }\end{array}$ & $\begin{array}{l}\text { B3 relative } \\
\text { to B4 }\end{array}$ & $\begin{array}{l}\text { B3 + B4 } \\
\text { Optimization }\end{array}$ & $\begin{array}{l}\text { B3+B4 relative } \\
\text { to B4 }\end{array}$ \\
\hline $\begin{array}{l}\mathrm{E}_{\mathrm{dg}} \text { [maximum } \\
\text { biodegradation } \\
\text { strain] }\end{array}$ & 0.32755 & 0.30256 & $8 \%$ & 0.32180 & $2 \%$ \\
\hline $\begin{array}{l}\mathrm{E}_{\text {Start }} \text { [Starting } \\
\text { Young's Modulus] }\end{array}$ & $1.3184 \mathrm{E} 5[\mathrm{~Pa}]$ & $1.3282 \mathrm{E}[\mathrm{Pa}]$ & $-0.7 \%$ & $1.2771 \mathrm{E} 5[\mathrm{~Pa}]$ & $3 \%$ \\
\hline $\begin{array}{l}\text { M [Young's Modulus } \\
\text { fitting parameter] }\end{array}$ & 1.4761 & 1.2774 & $13 \%$ & 1.4221 & $4 \%$ \\
\hline $\begin{array}{l}\text { N [Young's Modulus } \\
\text { fitting parameter] }\end{array}$ & 0.66542 & 0.50079 & $25 \%$ & 0.62977 & $5 \%$ \\
\hline $\begin{array}{l}\text { A [Poisson's ratio } \\
\text { fitting parameter] }\end{array}$ & 0.87698 & 0.95988 & $-9 \%$ & 0.88887 & $-1 \%$ \\
\hline $\begin{array}{l}\text { B [Poisson's ratio } \\
\text { fitting parameter] }\end{array}$ & 0.93234 & 0.99196 & $-6 \%$ & 0.93642 & $-0.4 \%$ \\
\hline$\tau$ [Relaxation time] & $1.4375 \mathrm{E}$ [s] & $1.4856 \mathrm{E}[\mathrm{s}]$ & $-3 \%$ & $1.4283 \mathrm{E} 7$ [s] & $0.6 \%$ \\
\hline RESULTING FIT & 0.41329 & 0.63439 & & 1.3844 & \\
\hline
\end{tabular}


Bundle 3's lack of response to the application of the fifth waste lift may be due to loading geometry, load sequencing, or material phenomenon. This unexpected lack of response to loading is also seen briefly with Bundle 4 during the application of the third waste lift, circa year 1.5 (Figures 39 and 43). The FEM model reacts to a larger magnitude of loading, where the field data shows a lesser response. The difference between the field data and the FEM model is corrected by the application of the fourth waste lift (circa year 2). It is possible that these waste lifts were not placed evenly, which would explain why B3 responds to lift 3 while B4 does not, and why B4 responds to lift 5 when B3 does not. However it is also equally likely that a thermal or hydraulic behaviour is influencing the material stiffness. This behaviour is not as problematic in the Marques-based model due to its separate mechanism for instantaneousresponse to loading, which allows the optimization in the Marques-based model to account for this response explicitly.

The viscoelastic model provided very good data matching during the construction phase of the landfill (Figures 37, 39, 41, 43). The viscoelastic model's curves have a better shape to match the field data, which is very apparent in the displacement graphs for both model types. This is a large improvement from the model's previous incarnation in McDonald et al. (2017). Much of the improvement is due to the alteration of the model`s instantaneous behaviour. Originally, the viscoelastic body was a threeparameter solid, with a linear spring handling the instantaneous response to loading, and a KelvinVoight body handling the time-dependent response. The linear spring behaviour was handled by the finite element response in the model, and controlled by the elastic material parameters, i.e. Young's Modulus and Poisson's ratio. These parameters were originally constant in McDonald et al. (2017). In updating the model, stress and strain dependency were introduced to these parameters in hopes of obtaining a less linear and rigid instantaneous response to loading. These improvements proved successful with the Marques-based model, and did help with viscoelastic model as well. Figure 44 depicts some results from the viscoelastic model at this stage. Compared to McDonald et al. (2017) it 
can be seen that stress/strain dependent parameters have greatly improved the fit, but the correspondence to the field data is still far from ideal.

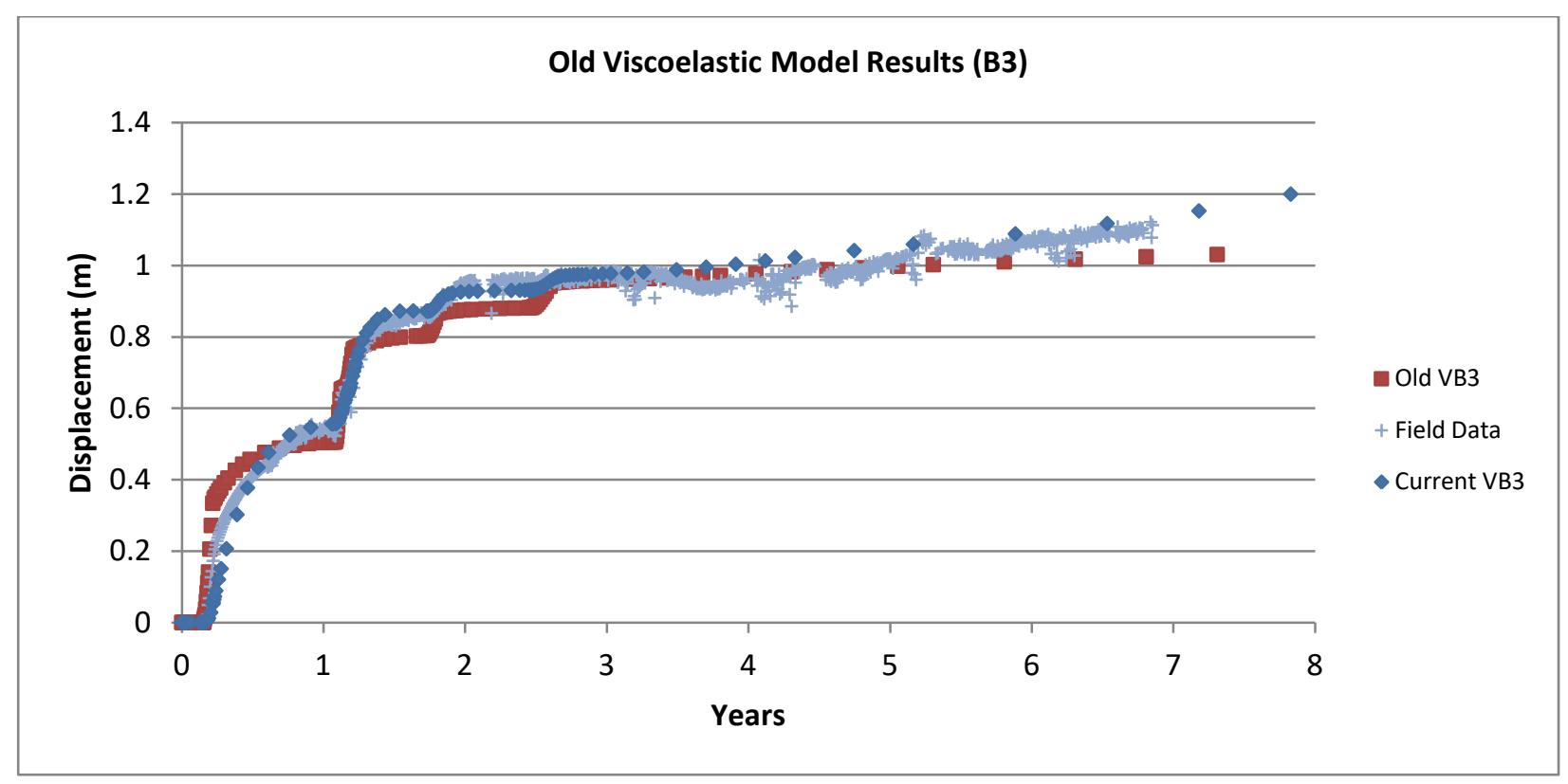

Figure 44: Old viscoelastic model with Bundle 3 displacement results

The old viscoelastic model is much more linear and rigid than the field data, resembling a 'staircase pattern' past the first waste lift. This pattern is the result of an over-dependence on the instantaneousresponse to loading from the initial spring, and a lack of activity with the time-dependent behaviours from the Kelvin-Voight body and the biodegradation function. The stress/strain dependency of the spring is quickly depleted in the first waste lift, leaving a more linear response in the upper lifts. Looking more closely at the response to first waste lift, the field data retains a very linear response and good match with the field data up to approximately. $0.15 \mathrm{~m}$ of displacement. The field data then shows a softer response, whereas the viscoelastic model remains linear to approximately $0.35 \mathrm{~m}$ of displacement, then starts softening. This is due to the starting stiffness of the initial spring in the model, controlled by $E_{\text {START }}$. In optimizing this starting stiffness, the resulting spring moduli were approaching zero to the effect of a non-significant initial spring in the three-parameter viscoelastic body. At this 
point, it was decided to remove the initial spring from consideration and change the rheological setup from a three-parameter solid to a single Kelvin-Voight body. Removing the initial spring (and therefore the separate explicit expression for an instantaneous settlement), is contrary to other similar settlement models for MSW, including Sowers (1973), Edil et al. (1990), Marques et al. (2003), and Babu et al. $(2010,2011)$. These models do not incorporate the slow placement of MSW for filling a landfill cell, such as the construction-equivalent loading featured in the two models proposed in this thesis. A slower loading process, which is more reflective of actual landfill operations, can be better modeled by using a time-dependent mechanism, such as a Kelvin-Voight body. Unfortunately, settlement field data for MSW which incorporates the filling and construction phase is not very common across literature.

Changing from an optimized three-parameter solid to an optimized single Kelvin-Voight body yielded the current viscoelastic model results seen in Figures $36-43$. The removal of the spring yielded a simpler model and much improved data matching overall, but introduced one small caveat: the instantaneousresponse to loading is now consistently more gradual - which is not perfectly consistent with the field data in the short term (i.e. first couple months after loading). Bundles 11 and 12 show the greatest impact of this effect at the application of the fifth waste lift (Figures 36, 38, 40, and 42). However, the more pertinent goals for data matching are the long-term settlement and the pre-closure settlement which this model achieves more than adequately. Arguably, the caveat of some dissonance in the short term is acceptable with improved long-term results.

Regarding the long-term behaviour, each parameter set performed almost equally well, with the B3+B4 parameter set performing slightly better at predicting the upper bundle elevation results for both the odd and even bundles, B3 results slightly underestimate the settlement, and B4 results slightly overestimate the settlement (Figures $36,38,40$, and 42 ). The long-term behaviour is dominated by mechanical creep, controlled by relaxation time, and the biodegradation-induced settlement, controlled 
by the maximum biodegradation strain. Each viscoelastic optimization produced a nearly identical maximum biodegradation strain (approximately 0.32). Because of the near identical biodegradation parameters in the viscoelastic optimization, it can be argued that the difference in long-term behaviour is reflective of the difference in relaxation time. Therefore, the best proposed relaxation time for this landfill comes from B3+B4 at 1.4283 E7 seconds (165.3 days) with a best maximum biodegradation strain at approximately 0.32 . Notably, the value of 0.32 is higher than typical values from literature, which normal range from 0.11 - 0.24 (Marques et al., 2003; Babu et al., 2010; Van Geel and Murray, 2015). However, these are only the best values proposed for the model in its current state. As discussed earlier, the value of $E_{D G}$ is subject to improvement if optimization could be performed on the upper bundles in addition to the lower bundles (due to the low biodegradation activity in the lower bundles). A change in the biodegradation term would in turn affect the parameters controlling mechanical creep. Regardless, the viscoelastic model achieved good correspondence with the field data without this change and its current parameters.

\subsection{Comparison of Marques-based Model and Viscoelastic Model}

Both the Marques-based and the viscoelastic model achieved good correspondence with the field data. Regarding the 'resulting fit' of each optimization run, the Marques-based model better matched the field data over the viscoelastic model. This strength of the Marques-based model is due to its explicit and separate expressions defined for the instantaneous response to loading and the time-dependent mechanical creep. Because the instantaneous and time-dependent behaviour are not linked, nor dependent on each other, each component of settlement can be adjusted without affecting the other components. This allows the parameters found in the Marques-based model to be highly flexible and to be optimized to match virtually any shape of waste settlement behaviour. In contrast, the viscoelastic model's spring-dashpot mechanism in parallel (the K-V body) controls both the instantaneous behaviour and mechanical creep. Optimizing the viscoelastic model's parameters thus requires balancing the effect 
on both components of settlement behaviour. Unfortunately, the explicit set-up of the Marques-based model yields a poor gradient and a rigid transition from the instantaneous behaviour to the timedependent behaviour. In contrast, the viscoelastic model produces settlement curves with gradients that are smooth and reflective of real waste settlement behaviour.

Both models encountered an unexpected stiffening pattern for Young's modulus with stress when optimizing against the field data of Bundle 3 . However, only a single parameter controlling that trend need to be constrained to yield normal behaviour. If we consider the constrained optimization results of Bundle 3, the optimized parameters obtained for each model were very consistent, with a low relative difference, from each field data set $(B 3, B 4$, and $B 3+B 4)$. As the $B 3+B 4$ optimizations were provided the most complete dataset for a single lift of waste, they would be the best overall parameters for generalized waste behaviour. This dataset also provided the best prediction for the settlement of the upper bundles. Table 8 compares the parameters from both models from this optimization.

Table 8: Average Parameters of Marques-based model and viscoelastic model optimizations

\begin{tabular}{|l|l|l|l|}
\hline & $\begin{array}{l}\text { Marques- based B3 + B4 } \\
\text { Optimization }\end{array}$ & $\begin{array}{l}\text { Relative Difference of } \\
\text { Viscoelastic Model to } \\
\text { Marques-based Model }\end{array}$ & $\begin{array}{l}\text { Viscoelastic B3 + B4 } \\
\text { Optimization }\end{array}$ \\
\hline $\begin{array}{l}\mathrm{E}_{\mathrm{dg}} \text { [maximum } \\
\text { biodegradation strain] }\end{array}$ & 0.23307 & $-38 \%$ & 0.32180 \\
\hline $\begin{array}{l}\mathrm{E}_{\text {Start }} \text { [Starting Young's } \\
\text { Modulus] }\end{array}$ & $4.0296 \mathrm{E} 5[\mathrm{~Pa}]$ & $1.2771 \mathrm{E} 5$ [Pa] \\
\hline $\begin{array}{l}\text { M [Young's Modulus fitting } \\
\text { parameter] }\end{array}$ & 1.3617 & $-4 \%$ & 1.4221 \\
\hline $\begin{array}{l}\mathrm{N} \text { [Young's Modulus fitting } \\
\text { parameter] }\end{array}$ & 0.61614 & $-2 \%$ & 0.62977 \\
\hline $\begin{array}{l}\text { A [Poisson's ratio fitting } \\
\text { parameter] }\end{array}$ & 0.92974 & $4 \%$ & 0.88887 \\
\hline $\begin{array}{l}\text { B [Poisson's ratio fitting } \\
\text { parameter] }\end{array}$ & 0.95758 & $2 \%$ & 0.93642 \\
\hline $\begin{array}{l}\boldsymbol{b} \text { [coefficient of mechanical } \\
\text { creep] }\end{array}$ & $3.7692 \mathrm{E}-6[1 / \mathrm{Pa}]$ & & $1.4283 \mathrm{E}]$ [s] \\
\hline $\begin{array}{l}\boldsymbol{c} \text { [rate constant for } \\
\text { mechanical creep] }\end{array}$ & $6.7216 \mathrm{E}-8[1 / \mathrm{s}]$ & & \\
\hline$\tau$ [Relaxation time] & & & \\
\hline
\end{tabular}


Table 8 shows consistency across both model types regarding the fitting parameters for Young's modulus and Poisson's ratio, with low relative differences of $2 \%$ and $4 \%$. These fitting parameters reveal consistent changes to the elastic material over time in both models. There is a large discrepancy between both models for the optimized value of $E_{\text {Start }}$, at a difference of $68 \%$. The Young's modulus for the viscoelastic model ( $127 \mathrm{kPa})$ is also noticeably smaller than the Marques-based model (403 kPa). Both values are within the typical ranges seen in literature : $50 \mathrm{kPa}$ to $800 \mathrm{kPa}$ (Sing and Flemming, 2008; Beaven and Powrie, 1995). However, a direct comparison of these two values for Young's modulus is not realistic; within the viscoelastic model the elastic material parameters are more involved with long-term behaviour, whereas the elastic material parameters are exclusively concerned with instantaneous behaviour in the Marques-based model.

\section{Conclusions and Recommendations for Future Work}

\subsection{Conclusions}

Two FEM mechanical models were developed in COMSOL Multiphysics to simulate and predict the settlement behaviour in engineered landfills. The first model was a modification of the work proposed by Marques et al. (2003), and the second, was a model based on the theory of viscoelasticity. Both models were developed with the goal of better representing the mechanical behaviour seen in MSW. The models made efforts to account for the three most distinct contributors to MSW settlement: (1) instantaneous response to load; (2) time-dependent mechanical creep; and (3) biodegradation-induced settlement. Both the Marques-based model and the viscoelastic model underwent parameter optimization using settlement field data obtained from instruments installed within a landfill in Ste. Sophie Quebec, Canada. 
The Marques-based model and the viscoelastic model both functioned well. Both matched the field data of the lower bundles well, and in turn predicted the displacement of the upper bundles with a high degree of accuracy. The improvements from the previous incarnations of the models seen in McDonald et al. (2017) are many and feature contributions that can be applied effectively to other MSW models. The inclusion of a construction-equivalent loading mechanism, that better replicates real waste placement patterns in the field, shows the instantaneous settlement term seen in the viscoelastic model may better be represented by a time-dependent settlement mechanism, like a Kelvin-Voight body. This Kelvin-Voight body also better depicts the shape of field waste behaviour. A new temperaturedependent biodegradation settlement component better separates time-dependent settlement behaviour by more accurately accounting for the delayed biodegradation settlement featured in frozen MSW. Another improvement was the introduction of a stress-dependent Young's Modulus and a straindependent Poisson's ratio. Both helped improve the deformation behaviour to be more reflective of the field data. Initially, only a stress-dependent Young's modulus was included, but the inclusion of both functions greatly improved the fit. The strain-dependent Poisson's ratio enabled the time-dependent strain behaviours (mechanical creep and biodegradation-induced strain) to have an impact on the MSW's elasticity. After optimization, both model types achieved consistent parameters controlling the Young's Modulus and Poisson's ratio, confirming that there are significant stress and strain dependencies within the deformation behaviour.

Young's modulus for both models are representative of values recorded in literature, although due to the variability of MSW, the range of moduli recorded is quite large. The Young's modulus for the viscoelastic model (127 kPa) is also noticeably smaller than the Marques-based model (403 kPa). However, a direct comparison of these two values for Young's modulus is not realistic; within the viscoelastic model the elastic material parameters are more involved with long-term behaviour, whereas 
the elastic material parameters are exclusively concerned with instantaneous behaviour in the Marquesbased model.

Aside from the parameters concerning mechanical creep, most of the parameters are consistent across both models. Correspondence between each modelling approach for the fitting parameters of the Young's modulus and Poisson's ratio suggest consistent stiffening and deformation behaviour across both models. For the viscoelastic model, the optimized $\mathrm{E}_{\mathrm{DG}}$ is higher than the Marques-based model, which could be a reflection on how the mechanical creep and biodegradation behaviour are balanced as long-term MSW behaviour. The new temperature-dependent biodegradation component, based off the work from Bequist (2017), did allow the model to more accurately separate the biodegradation behaviour by accounting for the delayed biodegradation effect seen in frozen MSW. The Marques-based model optimization predicted an $\mathrm{E}_{\mathrm{DG}}$ value that is consistent with the literature. Improving the $\mathrm{E}_{\mathrm{DG}}$ estimate will benefit from future monitoring as only approximately $50 \%$ of the biodegradation-induced settlement has occurred over the seven year record in the bundles used for optimization.

The Marques-model is very proficient in replicating the long-term magnitude of the field data's settlement. This strength comes from the model's three separate explicit expressions for evaluating waste behaviour. With three separate expressions, it can easily piece together an appropriate displacement pattern with sufficient parameter inputs. However, the explicitness of the Marques-based model also shows faults. While a good pattern match can be achieved, the resulting trend lines in the plotted settlement are notably rigid, akin to a stepwise function, with sharp transitions between each mode of stabilization, whereas actual waste behaviour would exhibit no rigidity in its gradients. This rigidity is more prominently seen at the transition from the instantaneous response to loading to the time-dependent mechanical creep and biodegradation processes. The explicit expressions also leave little room for linking between the stabilization modes, as each expression uses unique parameters. 
Having extra parameters also leads to a more complex model, which can hinder adaptability to other landfill sites when overly site-dependent parameters are involved. While it performs well at predicting long-term changes in elevation, in the short term, during the construction phase, the optimized parameters produce some significantly different patterns and do not match the displacement as well. This will mean limited accuracy of predicting pre-capping stabilization, which is a desirable feature of waste stabilization models.

The viscoelastic model is more effective at matching the shape of waste stabilization displacement trends compared to the Marques-based model. Because of this, it is performs well at predicting the displacement and behaviour of the waste profile during the construction phase of the landfill, particularly at the transition between instantaneous and time-dependent behaviour. The initial viscoelastic model seen in McDonald et al. (2017) featured similar linear rigidity to the Marques-based model. After the removal of its initial spring and reducing the viscoelastic behaviour to just a single Kelvin-Voight body, the viscoelastic model lost the accompanying rigidity that the Marques-based model features. The loss of this rigidity and initial spring however causes the viscoelastic model to lack an immediate displacement when undergoing loading - as the dashpot will always be active in the KelvinVoight body. Because of this, the viscoelastic model is not as accurate in matching the instantaneous response to loading for the upper bundles. The upper bundles sit upon a much larger volume of waste and are susceptible to a larger instantaneous compression under loading - and this larger compression is delayed by the dashpot in the viscoelastic model. For the Ste. Sophie landfill, this delay was only largely apparent in the top most waste lift. However, it is arguably more important that the stabilization model be more accurate at predicting the pre-capping displacement and long-term stabilization, than the displacement during the immediate-response. The simplicity of the viscoelastic model and its applicable theory also aids in its customization and linking with other behaviours. Within the built-in features of 
COMSOL Multiphyiscs viscoelasticity model, there are many customizations available for thermal effects, as is the case with other geotechnical software.

\subsection{Recommendations for Future Work}

Both models are suitable in their current form to provide a fair representation of the mechanical behaviour of solid waste. However, better stabilization understanding and more accurate results can be achieved with continued development of these models. Some improvements can be made to the specific dataset discussed in this thesis. Optimizations on additional bundle sets throughout the landfill profile would allow a better average of typical waste performance. Another improvement would be creating more accurate loading patterns. The inclusion of a compaction force during lift placement could replicate the effect made by landfill operators. The model created within this thesis was for instrumented bundles near the edge of a landfill. Being so close to the edge is likely to have an effect on the stress distribution. Additionally, the loading of the fifth waste lift could be more accurate, as it was placed in more stages than a typical lift, as the result of a neighbouring landfill cell being constructed next to, and then on top of, the existing landfill cell. A three-dimensional model could achieve this complexity. Additionally, there is GIS data for the surface profile of the landfill (as is discussed in Appendix B); this GIS data could be used to create 3D meshes, to improve the 2-D geometry, or to optimize the displacement of the surface of the model previously proposed.

The most desirable improvement is to link these mechanical models in COMSOL Multiphysics to the existing thermal model developed. Having internal temperature profiles in the same mechanical model will lead to more accurate biodegradation behaviour, with the biodegradation being mapped to temperature gradients as opposed to arbitrary 'bundle layers'. The 'bundle' layers in the current model are controlled by only one set of instrument bundles and generalized the temperature profile across this horizontal layer. The temperature profile will also allow applying appropriate mechanical properties to 
the frozen waste, which should behave much like a frozen soil. Tracking the temperature will also allow the behaviour of frozen waste to be further explored, of which there is little information about available in literature. The viscoelastic model in particular will be interesting, as traditional viscoelastic materials are highly affected by temperature. Should a hydraulic component be developed as well, its inclusion will further improve the mechanical behaviour, with an exploration of the effect of leachate in landfills and its comparison to saturated soil mechanics. In short, a fully coupled mechanical-thermal-hydraulicbiologic model would allow for a richer understanding of MSW stabilization behaviour. A better understanding of MSW stabilization will in turn yield better landfill operation practices, such as an improved sequencing of frozen and warm waste lifts; create more efficient and profitable landfills; and lessen the environmental impact of landfills. 


\section{References}

Babu Sivakumar, G. L., Reddy, K. R., Chouskey, S. K., and Kulkarni, H. S. (2010). “Prediction of long-term municipal solid waste landfill settlement using constitutive model." Pract. Period. Hazard. Toxic Radioact. Waste Manage., 14(2), 139-150.

Babu Sivakumar, G. L., Reddy, K. R., Chouskey, S. K. (2011). Parametric study of MSW landfill settlement model. Waste Manage. 31 (6), 1222-1231.

Bareither, C. A. (2010). "Compression behavior of solid waste." Ph.D. dissertation, Univ. of WisconsinMadison, Madison, WI.

Bareither, C.A. (2012). "Compression behaviour of Municipal Solid Waste: Immediate Compression." Journal of Geotechnical and GeoEnvironmental Engineering, ASCE 128 (12), 1047 - 1062.

Bareither, C.A., Kwak, S. (2015). Assessment of municipal solid waste settlement models based on fieldscale data analysis. Waste Manage. Volume 42, August 2015, pp. 101-117.

Beaven, R.P., Powrie, W. (1995). Hydrogeological and geotechnical properties of refuse using a large scale compression cell. Proc. 5th International Waste Management and Landfill Symposium, Sardinia, pp. 745-760.

Berquist, C. (2017). The impacts of temperature and thermal properties on municipal solid waste stabilization. M.A.Sc. Thesis, Carleton University, Ottawa, Canada.

Bjarngard, A., Edgerm L. (1990). Settlement of municipal solid waste landfills. In: Proc. 13th Int. Madison Waste Conf. University of Wisonsin-Madison, Madison, WI, USA, pp.192-205.

Bonany, J.E. (2012). Heat budget for an anaerobic bioreactor landfill in Sainte-Sophie, Quebec, Canada. M.A.Sc. Thesis, Carleton University, Ottawa, Canada.

Boussinesq, J. (1885). Application des Potentiels a L'etude de L'equilbre et du Movement des Solids Elastiques, Gauthier-Villars, Paris.

Canada Environnemental Protection Act, 1990. Ontario Regulation 232/98 : Landfilling Sites. Last amendment: 268/11.

Chen, Y., Ke, H., Fredlund,D.G., Zhan, L., Xie, Y. (2010). Secondary compression of municipal solid wastes and a compression model for prediciting settlement of municipal solid waste landfills. J. Geotech. Geoenviron, Eng. 136 (5), 706-717.

Chen, K.S., Chen, R.H., Liu, C.N. (2012). Modeling municipal solid waste landfill settlement. J.Environ. Eng. Sci. 66 (8), 2301-2309.

COMSOL Multiphysics Reference Manual, version 5.3 (September 2017). COMSOL, Inc, www.comsol.com.

Doyle, J.T. (2015). A quasi-mechanical-biological model of the Ste-Sophie, QC Landfill. M.A.Sc. Thesis, Carleton University, Ottawa, Canada. 
Edil, T.B., Ranguette, V.J., Wueliner, W.W. (1990). Settlement of municipal refuse. In: Landva, A.O., Knowles, G.D. (Eds.), Geotechnics of Waste Fills-Theory and Practice, STP 1070. ASTM, West Conshohocken, PA, USA, pp. 225-239.

El-Fadel, M., Khoury, R. (2000). Modeling settlement in MSW landfills: a critical review. Crit. Rev. Environ, Sci. Technol.30 (3), 327-361.

Flügge, W. (1975). Viscoelasticity, 2nd edition. Springer-Verlag, New York/Heidelberg/Berlin, Germany.

Gibson, R.E., Lo, K.Y. (1961). A theory of soils exhibiting secondary compression. Acta Polytech. Scand. C10, 1-15.

Gourc, J.P., Staub, M.J., Conte, M. (2010). Decoupling MSW settlements into mechanical and biochemical processes - modeling and validation on largescale setups. Waste Manage. 30 (8-9), 15561568.

Hanson, J. L., Yesiller, N., Onnen, M.T., Liu, W.-L., Oettle, N.K., Marinos, J.A. (2013). Development of numerical model for predicting heat generation and temperatures in MSW landfills. Waste Manage. 33(10), 1967-2144.

Hettiarachchi, C.H., Meegoda, J.N. Hettiaratchi, J.P.A. (2007). Prediction of Waste Settlement in Bioreactor Landfills Incorporating Waste Biodegradation. ASCE Geotechnical Special Publication, p.163, ISBN 10 \# 078440897.

Hudson, A.P., White, J.K., Beaven, R.P., Powrie, W. (2004). Modeling the compression behavior of landfilled domestic waste. Waste Manage. 24 (3), 259-269.

Hossain, M.S., Gabr, M.A. (2005). Prediction of municipal solid waste landfill settlement with leachate recirculation. In: Proc. Geo-Frontiers, GSP No. 142, Waste Containment and Remediation. ASCE, pp. 10-14.

Janbu, N. (1963). Soil Compressibility as Determined by Oedometer and Triaxial Tests, European Conference on Soil Mechanics and Foundation Engineering, Wiesbaden, Germany, Vol. 1, pp. 19-25.

Lakes, R.S. (2009). Viscoelastic Materials. Cambridge University Press. ISBN 9780521885683.

Lackner, R., Pichler, C., Kloiber, A. (2008). Artificial Ground Freezing of Fully Saturated Soil: Viscoelastic Behavior. Journal of Engineering Mechanics, vol. 134/no. 1, pp. 1-11.

Li, D., Fan, J., Wang, R. (2011) Research on Visco-Elastic-Plastic Creep Model of Artificially Frozen Soil Under High Confining Pressures, Cold Regions Science and Technology, vol. 65/no. 2, pp. 219-225.

Ling, H.I., Leshchinsky, D., Yoshiyuki, M., and Toshinori, K. (1998). Estimation of municipal solid waste landfill settlement, J. Geotech. and Geoenviron. Eng., 124(1), 21-28.

Liu, C., Chen, R., Chen, K. (2006). Unsaturated consolidation theory for the prediction of long-term municipal solid waste landfill settlement. Waste Manage. 24, 80-91.

Lobo A, López A, Cobo N and Tejero I. (2008). Simulation of municipal solid waste reactors using Moduelo. Proceedings of the 1314 Waste Management \& Research 29(12) Institution of Civil Engineers, Waste and Resource Management 161(3): 99-104. 
Machado, S.L., Carvalho, M.F., Vilar, O.M. (2002). Constitutive model for municipal solid waste. Journal of the Environmental Engineering, ASCE 128 (11), 940-951.

Machado, S.L., Vilar, O.M., Carvalho, M.F. (2008). Constitutive model for long term municipal solid waste mechanical behaviour. Computers and Geotechnics 35, 775-790.

Marques, A.C.M.,Filz, G.M., Vilar, O.M. (2003). Composite compressibility model for municipal solid waste. J. Geotech. Geoenvrion. Eng. 129 (4), 372-378.

Marques, S., Creus, G. (2012). Computational Viscoelasticity, Springer-Verlag, Heidelberg/Berlin, Germany.

McBean, E.A., Rovers, F.A., Farquhar, G.J. (1995). Solid Waste Landfill Engineering and Design. Prentice Hall PTR, Englewood Cliffs, New Jersey 07632.

McDonald, R.P., Van Geel, P.J., Kenny, S. (2017). Predicting the Mechanical Behaviour of Solid Waste using Numerical Methods in Landfill Applications. Paper presented at GeoOttawa 2017 Conference, Ottawa, Canada.

McDougall, J. (2008). Landfill modelling challenge: HBM model predictions. Proceedings of the Institution of Civil Engineers, Waste and Resource Management 161(4): 147-153.

Megalla, D. (2015). Heat Transfer model for an engineered landfill in Sainte-Sophie, Quebec, Canada. M.A.Sc. Thesis, Carleton University, Ottawa, Canada.

Newmark, N.M. (1935). Simplified Computation of Vertical Pressures in Elastic Foundations, University of Illinois Engineering, Experiment Station, Circular No. 24, Illinois.

Park, H.I., Lee, S.R. (1997). Long-term settlement behavior of landfills with refuse decomposition. J. Res. Manage. Technol. 24 (4), 159-165.

Qian, X., Koerner, R. M., and Gray, D. H. (2002). Geotechnical aspects of landfill design and construction, Prentice Hall, Upper Saddle River, NJ.

Ramaiah, B.J., Ramana, G.V. (2017). Study of stress-strain and volume change behavior of emplaced municipal solid waste using large-scale triaxial testing. Waste Manage. 63 (2017) 366-379.

Sharma, H.D., Reddy, K.R. (2004). Geoenvironmental Engineering: Site Remediation, Waste Containment and Emerging Waste Management Technologies. John Wiley \& Sons, Inc., Hoboken, NJ.

Simões, G.F., Catapreta, C.A.A. (2013). Monitoring and modeling of long-term settlements of an experimental landfill in Brazil. Waste Manage. 33 (2), 420-430.

Singh, M.K. and Flemming, I.R. (2008). Estimation of the mechanical properties of MSW during degradation in a laboratory compression cell. Geotechnical Special Publication, ASCE, 177: 200-207.

Sing, M.K. (2008). Characterization of stress-deformation behaviour of municipal solid waste. PhD Philosophy Thesis, University of Saskatchewan, Saskatoon, Canada.

Sowers, G. F. (1968). Foundation problems in sanitary landfills, J. Sanitary Eng. Div., ASCE, 94(1),103116. 
Sowers, G.F. (1973). Settlement of waste disposal fills. In: Proc. 8th Int. Conf. on Soil Mech, and Found, Engr., vol.22, Balkema, Rotterdam, pp.207-210.

Terzaghi, K. (1943). Theorectical Soil Mechanics [M]. New York: Wiley, 345-398.

Van Geel, P.J., Murray , K.E. (2015). Simulating settlement during waste placement at a landfill with waste lifts placed under frozen conditions. Waste Manage. 46 (2015) 352-361.

White, J.K. (2008). The application of LDAT to the HPM2 challenge. Proceedings of the Institution of Civil Engineers, Waste and Resource Management 161(4): 137-146.

White, J.K., Beaven, R.P. (2013). Developments to a landfill process model following its application to two landfill modeling challenges. Waste Manage. 33 (10), 1969-1981.

White, J.K., Nayagum, D., Beaven, R.P. (2014). A multi-component two-phase flow algorithm for use in landfill processes modeling. Waste Manage. 34 (2014), 1644-1656.

Yazdani, R., Kieffer, J., Sananikone, K., Augenstein, D. (2006). Full scale bioreactor landfill for carbon sequestration and greenhouse emission control, final report, Award No. DE-FC26-01NT41152, U.S. Department of Energy, Washington, DC. 
8 APPENDIX A: Temperature Data

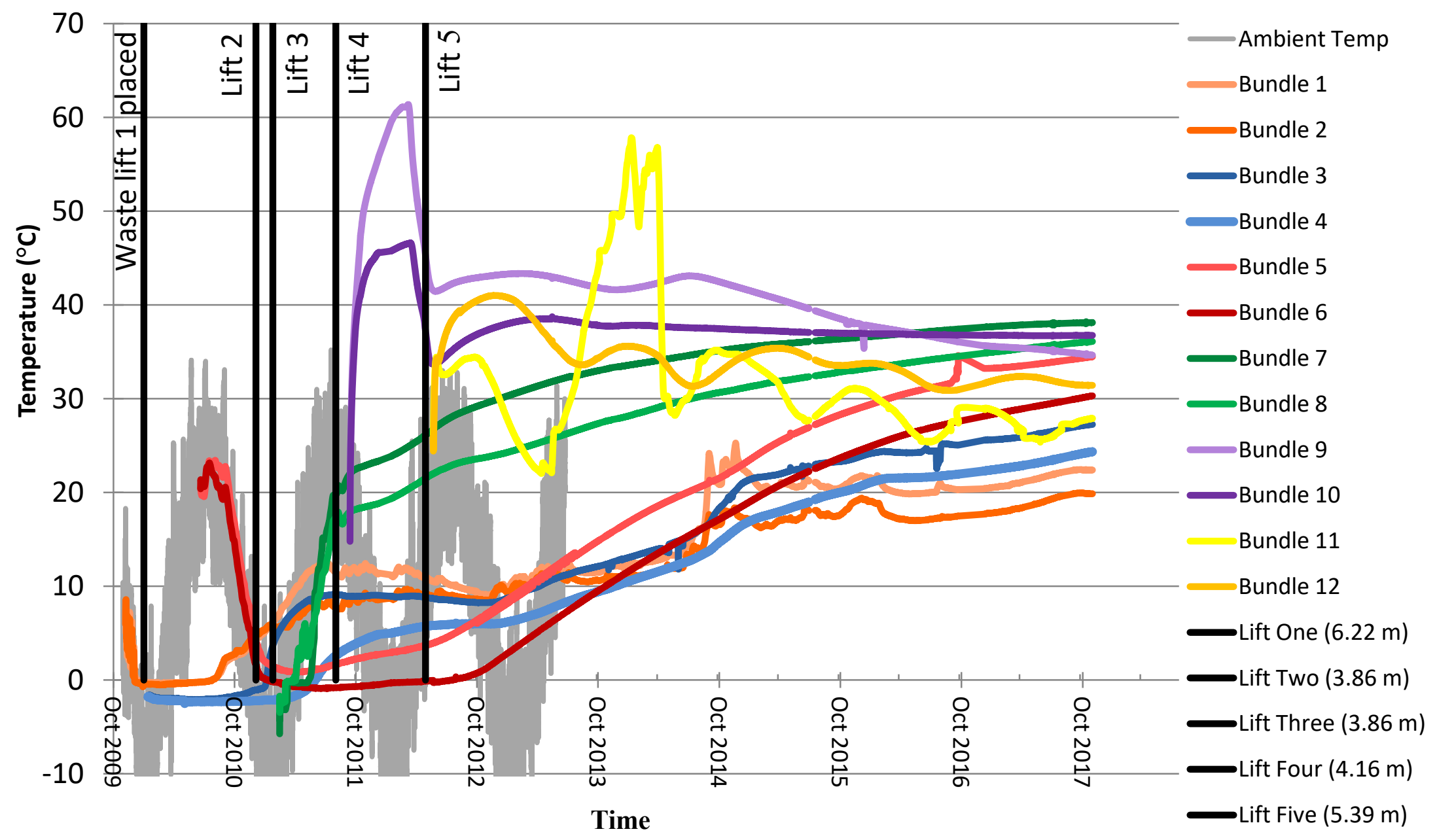

Figure 45: Ste. Sophie Temperature Data 


\section{APPENDIX B: GIS Work and Validation of TEPC Data}

\subsection{Problem Statement}

The following work was done near the beginning of the author's research, before the scope of the thesis had been determined. The goal of the work presented here was to validate and/or clarify the data recorded from the total earth pressure cell (TEPC) on each of the 12 instrument bundles installed within the Ste. Sophie Landfill in Quebec, Canada.

Unlike the other data sets (settlement, temperature, etc.) the results achieved from the TEPC were less than favourable. This data can be seen in Figure 46. While the expected trends were still present in the data, i.e. increasing stress with waste placement, the recorded magnitudes were off by an estimated factor of approximately 1.5-2, once compared to typical results of the same category. Additionally, many additional unexpected trends appear in the data, such as sudden changes in pressure without recorded waste being placed. Because the instrument bundles were installed at the edge of a cell in the landfill in Zone 4, it was thought that the construction of another cell in the neighboring Zone 5 may be having significant effects on the results in the instrument bundles. Notably, Zone 5 does end up overlapping and building at a taller height than Zone 4, so this concern was not unfounded. Investigating this behaviour would also provide insight on whether or not a FEM model incorporating one-dimensional settlement behaviour would be applicable - perhaps two-dimensional or three-dimensional behaviour would need to be incorporated.

Figure 46 includes black vertical bars to indicate the placement of the twelve instrument bundles. A red vertical bar also shows the parking of a heavy truck directly over the instrument bundles for 10 minutes. This truck placement was not a mistake made by landfill operators, but a request made by the research team. After the installation of Bundles 3 and 4 , it was noted that Bundles 1 and 2 were recording large 
increases in vertical stress, much more than anticipated, and increasingly off pattern compared to the results being recorded with Bundles 3 and 4. To investigate, the research team requested a large truck be parked overtop the bundles for a short while; this would provide more evidence on whether instrument failure had occurred, or some other phenomenon. After the truck was removed, the recorded stresses significantly dropped in magnitude. It was theorized that the weight of the truck broke up a large chunk of frozen waste and ice that was causing the irregular values. The concept of stiff bodies in a soft medium acting as locations of concentrated stress was later explored by another student to further explore the event.

\subsection{Proposed Solution}

To investigate the reliability of the Ste. Sophie data set, the TEPC results could not be directly compared to the trends from the other data sets recorded from the instrument bundles, as they could be subject to the same outside influences/errors. The proposed solution was to utilize survey data taken at the surface of the landfill at each quarter and use that information to investigate the increase in vertical stress from changes in waste height from one quarter to the next. The survey data available ranged from simple point data early in the landfill's construction, to highly detailed Lidar point clouds later on. This data was then used to generate three-dimensional surface profiles using the computer program ArcGIS for each quarter. A sample of these profiles during the construction of Zone 5 can be seen in Figure 4752. 


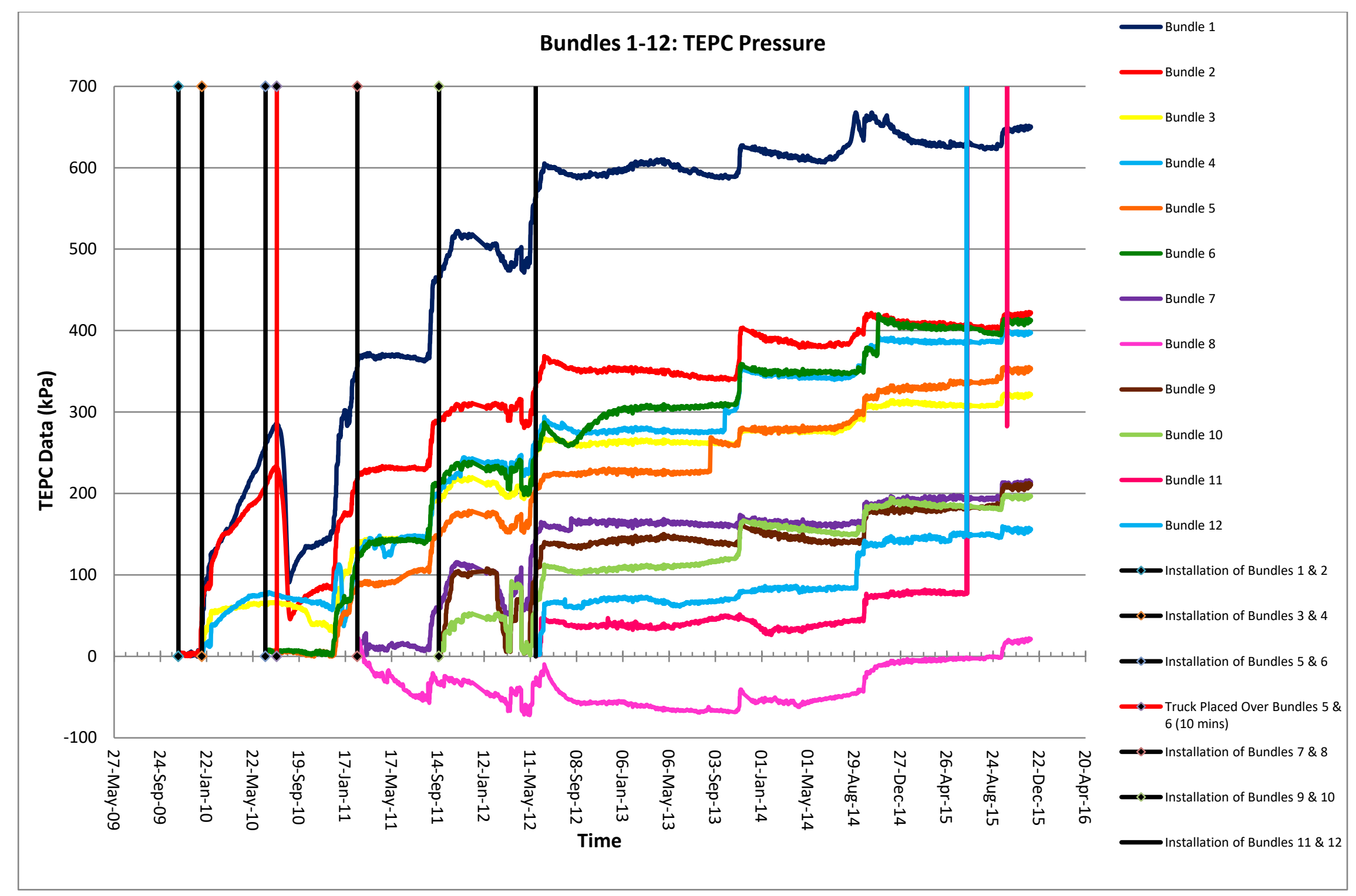

Figure 46: TEPC Field Data 

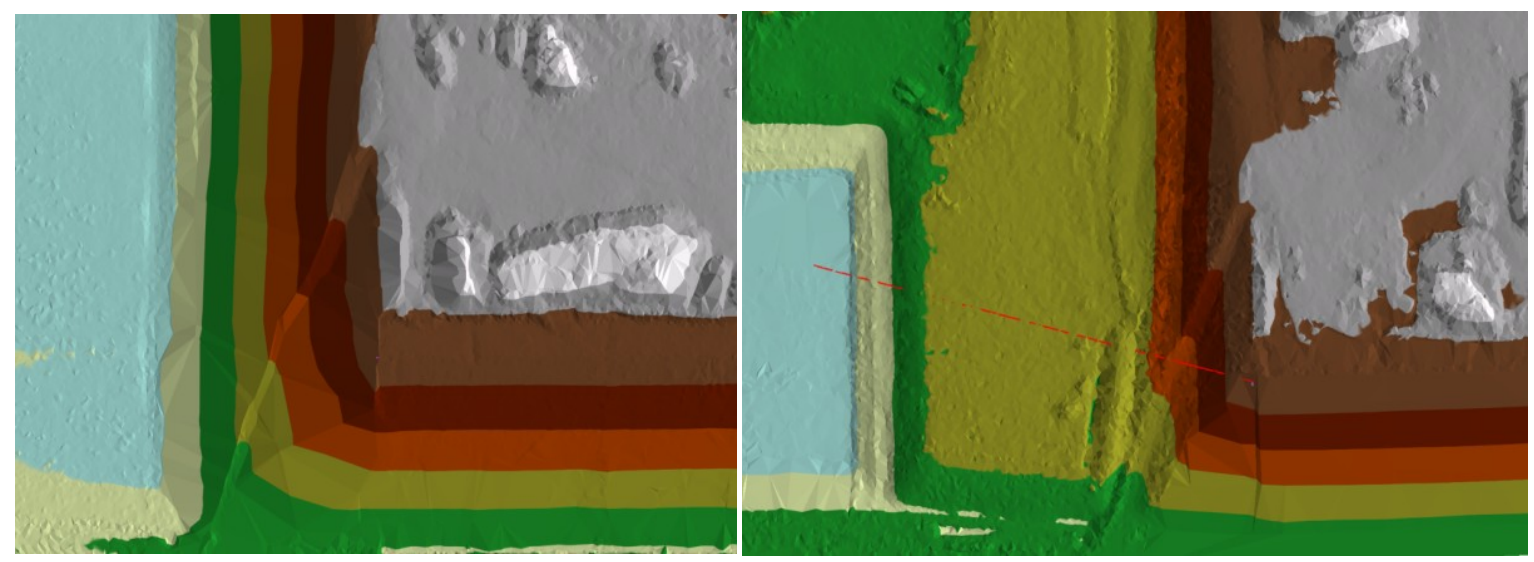

Figure 47: GIS Surface in June 2012

Figure 48: GIS Surface in July 2013
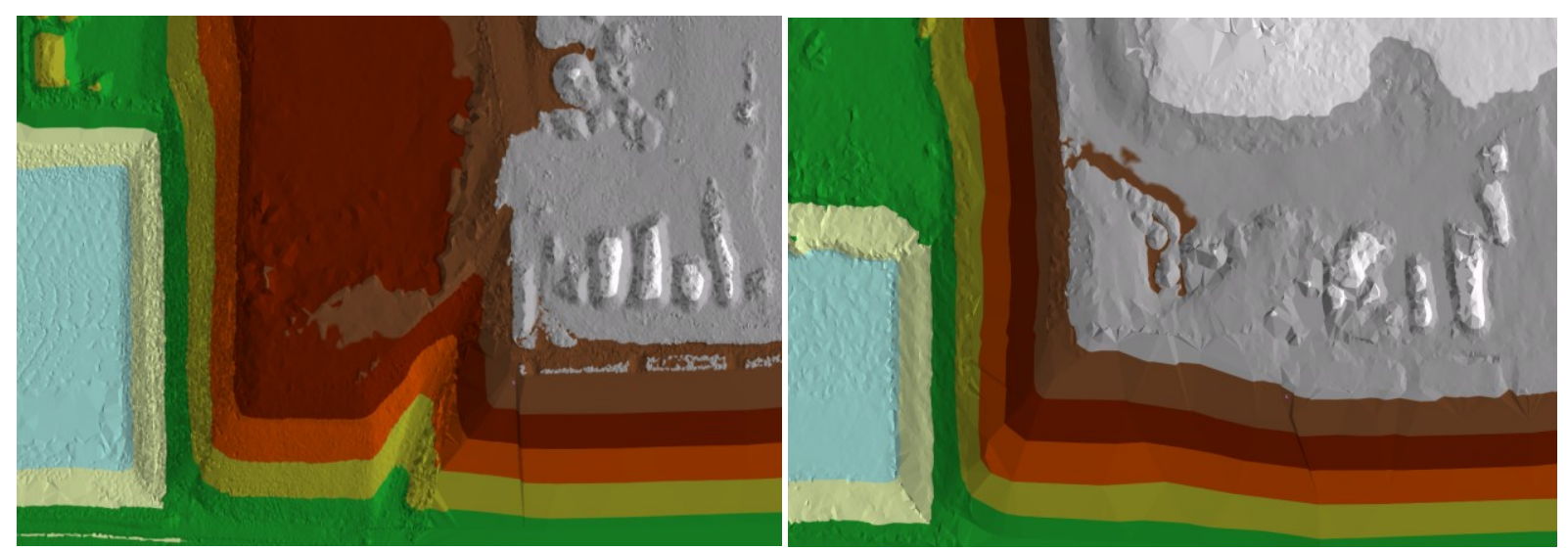

Figure 49: GIS Surface in September 2013

Figure 50: GIS Surface in December 2013
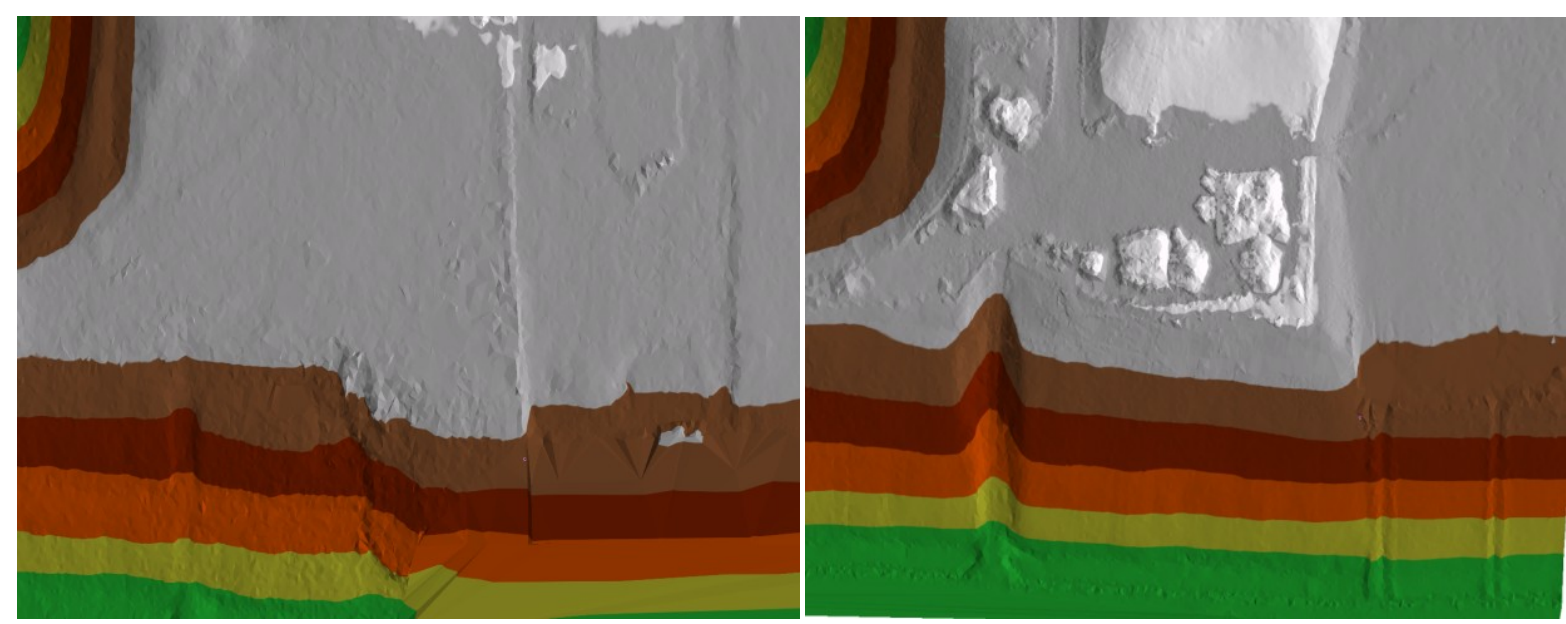

Figure 51: GIS Surface in September 2014

Figure 52: GIS Surface in December 2014 
Using these three-dimensional waste profiles, it was easy to visualize the placement of waste lifts and interpolate the elevations of the newly placed waste. Initially, the plan for these surface profiles was to compare them directly in ArcGIS and compute the changes in the surface from quarter to quarter to generate an exact volume of waste placed between those two surveys. Using this data, and the average density of waste, the vertical change in stress could be calculated. However, while this methodology seemed by all purposes correct, the results achieved from this method were unfavourable. In its stead, a more simplified approach was adopted. By comparing surface profiles, the additional waste could be idealized as three-dimensional rectangular cuboids. Combined with the average density of the waste, these cuboids would be equivalent to rectangular footings placed on the existing waste from the previous quarter and their contribution to vertical stress at the instrument bundles could be calculated through a simple manipulation of Boussinesq's solution (Boussinesq, 1885) for stress under a point load.

Boussinesq's solution for stress under a point load can be integrated to accommodate current situation: the increase in vertical stress under a uniformly rectangular area. The integration has been previously done and published by Newmark (Newmark, 1935) (see Equation B-1) and provides an expression for the vertical stress at a specified depth under the corner of a rectangular uniformly loaded area.

Equation B-1

$$
\Delta \sigma_{\mathrm{z}}=\frac{\mathrm{q}}{4 \pi}\left(\frac{2 \mathrm{BLz}\left(\mathrm{B}^{2}+\mathrm{L}^{2}+2 \mathrm{z}^{2}\right)}{\left(\mathrm{B}^{2}+\mathrm{z}^{2}\right)\left(\mathrm{L}^{2}+\mathrm{z}^{2}\right) \sqrt{\left(\mathrm{z}^{2}+\mathrm{B}^{2}+\mathrm{L}^{2}\right.}}+\tan ^{-1}\left(\frac{2 \mathrm{BLz} \sqrt{\left(\mathrm{z}^{2}+\mathrm{B}^{2}+\mathrm{L}^{2}\right.}}{-\mathrm{B}^{2} \mathrm{~L}^{2}+\mathrm{z}^{2}\left(\mathrm{~B}^{2}+\mathrm{L}^{2}\right)+\mathrm{z}^{4}}\right)\right)
$$

where $\Delta \sigma_{z}$ is the increase in vertical stress

$\mathrm{q}$ is the applied surface stress

$B, L$ are footing dimensions

$z$ is the depth below the footing 
The above equation was used alongside a series of interpolated cuboids interpolated from the additional waste placed each quarter. The surface stress was derived from the waste density (which was taken as $1000 \mathrm{~kg} / \mathrm{m}^{3}$ ) and the height of cuboids. The height of the cuboids was derived from the average height of the waste placed found using ArcGIS interpolated surface profiles. When there were significant differences in height in one area, additional cuboids would be defined. For waste that was placed at a distance, where its defined rectangular area would not have a corner overlapping the instrument bundles, the concept of superposition was used to evaluate the waste's effect at a distance. This methodology required the waste be idealized as homogeneous, isotropic, and semi-infinite; all of these assumptions are far from true for MSW, but would allow for a better understanding of the stress behaviour.

\subsection{Results}

Using Newmark's integration of Boussinesq's solution, the effective vertical stress from each quarter was found for all 12 instrument bundles at each of their respective depths. The depth of the instrument bundles were determined from the elevations recorded for each bundle from the research work done by Dina Megalla (Megalla, 2012). The "Boussinesq" solution in comparison to the field data can be seen below in Figure 53. The Boussinesq solution appears as dotted trend lines and is measured on the right axis, whereas the field data is measured on the left axis. 


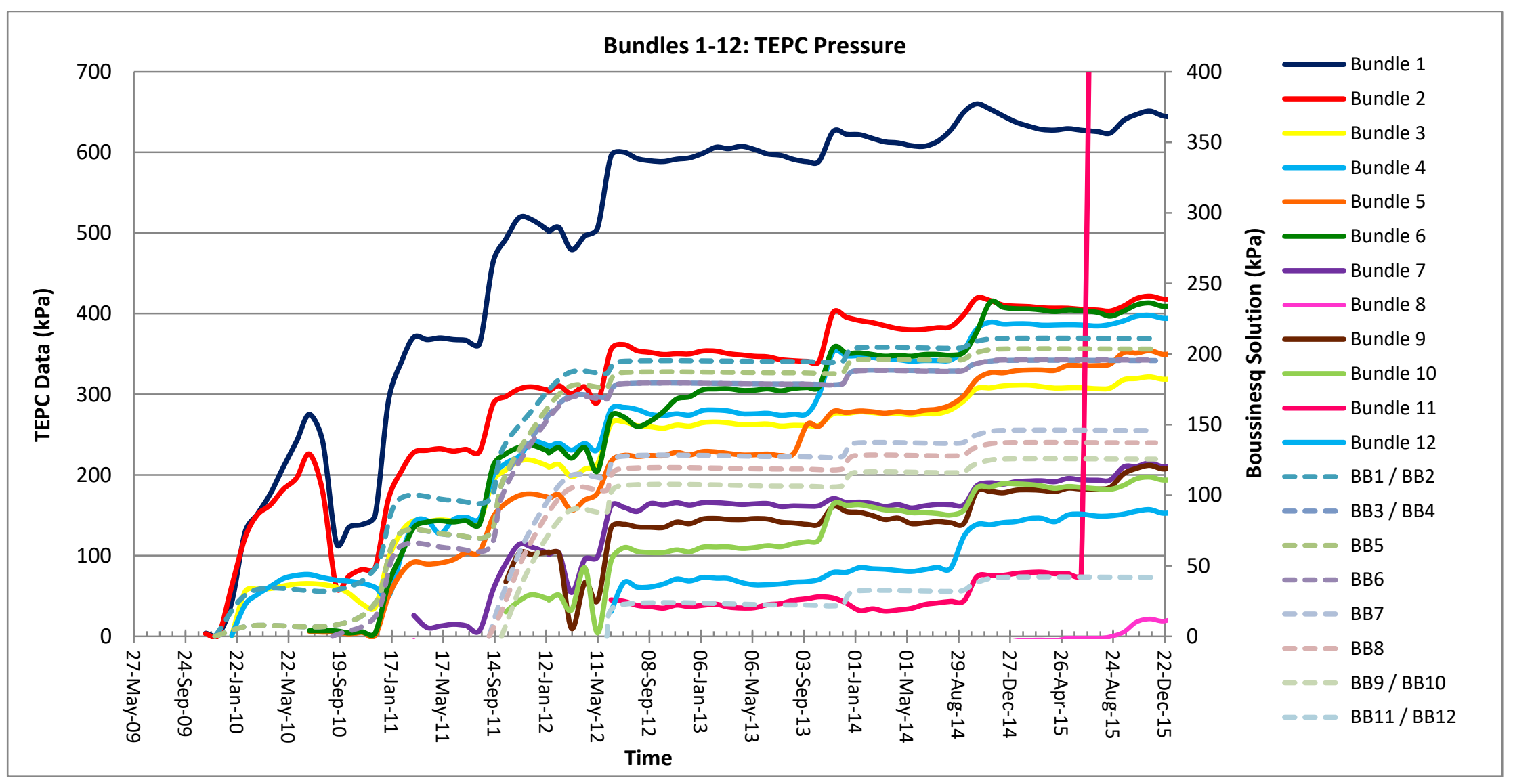

Figure 53: TEPC Field Data versus Boussinesq Solution Results 
The Boussinesq solution provided adequate results. Trend lines from the new solution matches those seen with the field data, as seen with the increase in stress at each lift placement. The new solution also provides a fair estimation as to what the field stress ought to realistically be, albeit a basic estimate. Comparing the Boussinesq results from Figure 53 to the stress profile created by the viscoelastic model in COMSOL multiphysics in Figure 54 shows comparable results. Boussinesq's Bundles 11 and 12 at circa $40 \mathrm{kPa}$ (Figure 53) seem consistent with the stress profile in the top most waste lift in Figure 54, as does the intensities of Boussinesq's Bundles 1 and 2 at $210 \mathrm{kPa}$ seem to fit in the lower most waste lift in Figure 54.

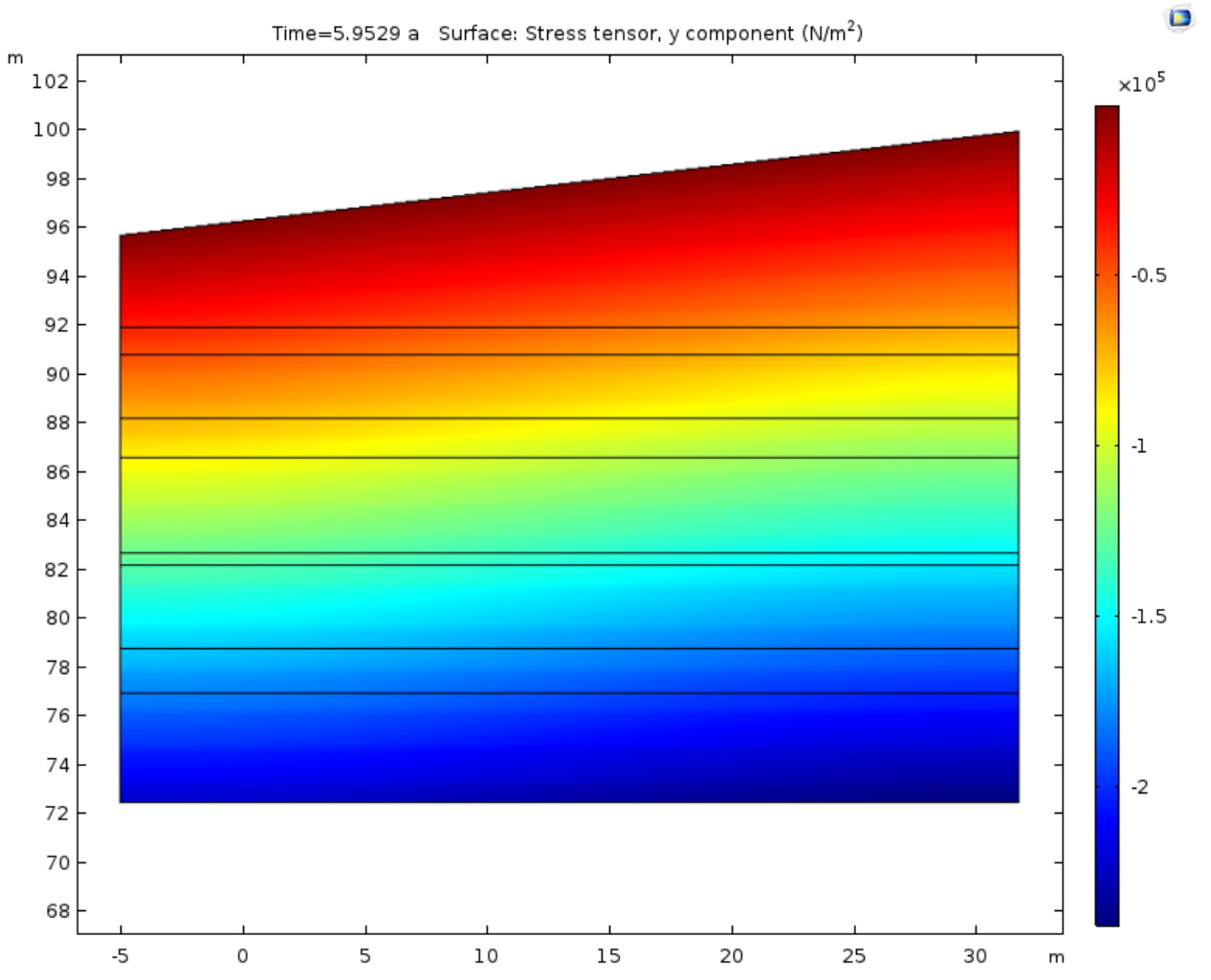

Figure 54: Stress profile from Viscoelastic Model for Ste. Sophie landfill

The most interesting discovery from the Boussinesq solution was the sensitivity of vertical stress increases at the instrument bundles versus the distance of the placed waste. It was worried that the construction of neighbouring zone 5 would have a significant effect on the vertical stresses recorded in 
zone 4. The impact of vertical stress from a loading typically follows a bulbous pattern; if any of the instruments should fall within lateral reach of zone 5's stress bulb, it would mean that a more detailed model, beyond one-dimensional loading, would be required to accommodate the additional complexity of the situation in the field.

Fortunately, via the Boussinesq calculations, the majority of the waste placed in zone 5 had minimal impact on the stresses recorded from the TEPC. Despite the large magnitude of waste placed, the distance from the waste to the instruments (for the most part) was sufficient. The final construction of zone 5 did end up overlapping the existing waste in zone 4, and as a result, additional waste was placed over the instrument bundles and there was some slope modifications as well. Only the waste placed directly overtop or significantly nearby the instrument bundles ended up producing a noticeable impact resulting from zone 5 . These results indicate that modelling simple one-dimensional behaviour would be sufficient. When waste material was placed nearby the bundles, two notable periods of significant stress increase occurred across all 12 bundles resulting from zone 5's construction : firstly between September 2013 to January 2014; and secondly circa October 2014. The effect of zone 5 on these stress spikes can be seen more clearly in Figure 55 and 56.

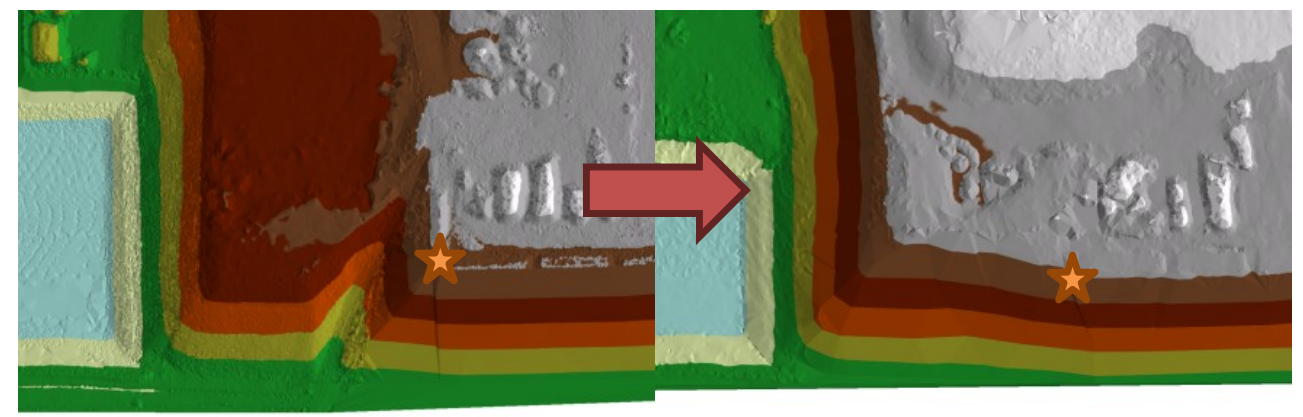

Figure 55: September 2013 - December 2013 (Instrument bundles marked by star) 


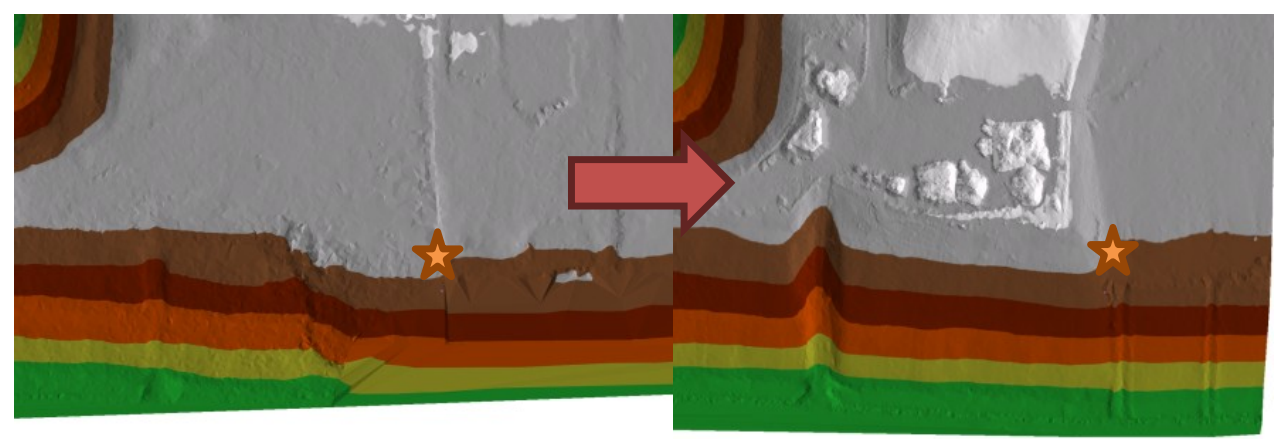

Figure 56: September 2014 - December 2014 (Instrument bundles marked by star)

The prevalence of these stress irregularities being strictly local, led to further investigation into other irregularities seen in the TEPC dataset. There are a number of dips and rebounds seen in the TEPC dataset, and these are theorized to be the result of local mounding. The GIS surface profiles would occasionally reveal tall piles mounded around the landfill, with the pile disappearing in the next quarter's survey. Occasionally, these piles would be placed directly overtop the instrument bundles. These are likely piles of contaminated soil which was used as daily cover soil and was stored as piles near the working face. That would explain the existence and removal of the piles, which in turn provides an explanation for some of the stress variance and rebound.

\subsection{Conclusions}

The survey data and GIS surface profiles offers a point of reference to explain the trends and irregularities seen in the TEPC dataset. It enabled a rough estimate of the true magnitude of stress being recorded via use of the Boussinesq formulation. The Boussinesq solution results were also favourable in matching and revealing trends in stress behaviour. The sensitivity of placement distance on vertical stresses allows the argument for simple one-dimensional settlement models to simulate waste behaviour with this particular dataset obtained from Ste. Sophie, Quebec, Canada. The results show even a smaller loading placed (even temporarily) over or near the instrument bundles had greater 
significance than a large MSW lift placed at a distance. More refined and accurate solutions could be obtained if the Boussinesq methodology was improved to incorporate the slopes of the landfill.

The potential applicability of the GIS dataset has only just been tapped. Other experiments with the dataset have shown it is possible to use the dataset to create 3D meshes in COMSOL Multiphysics (Figure 57). Using these 3D surfaces could allow for more precise loading schemes, or optimized solutions based on the actual change of each 3D surface from quarter to quarter.

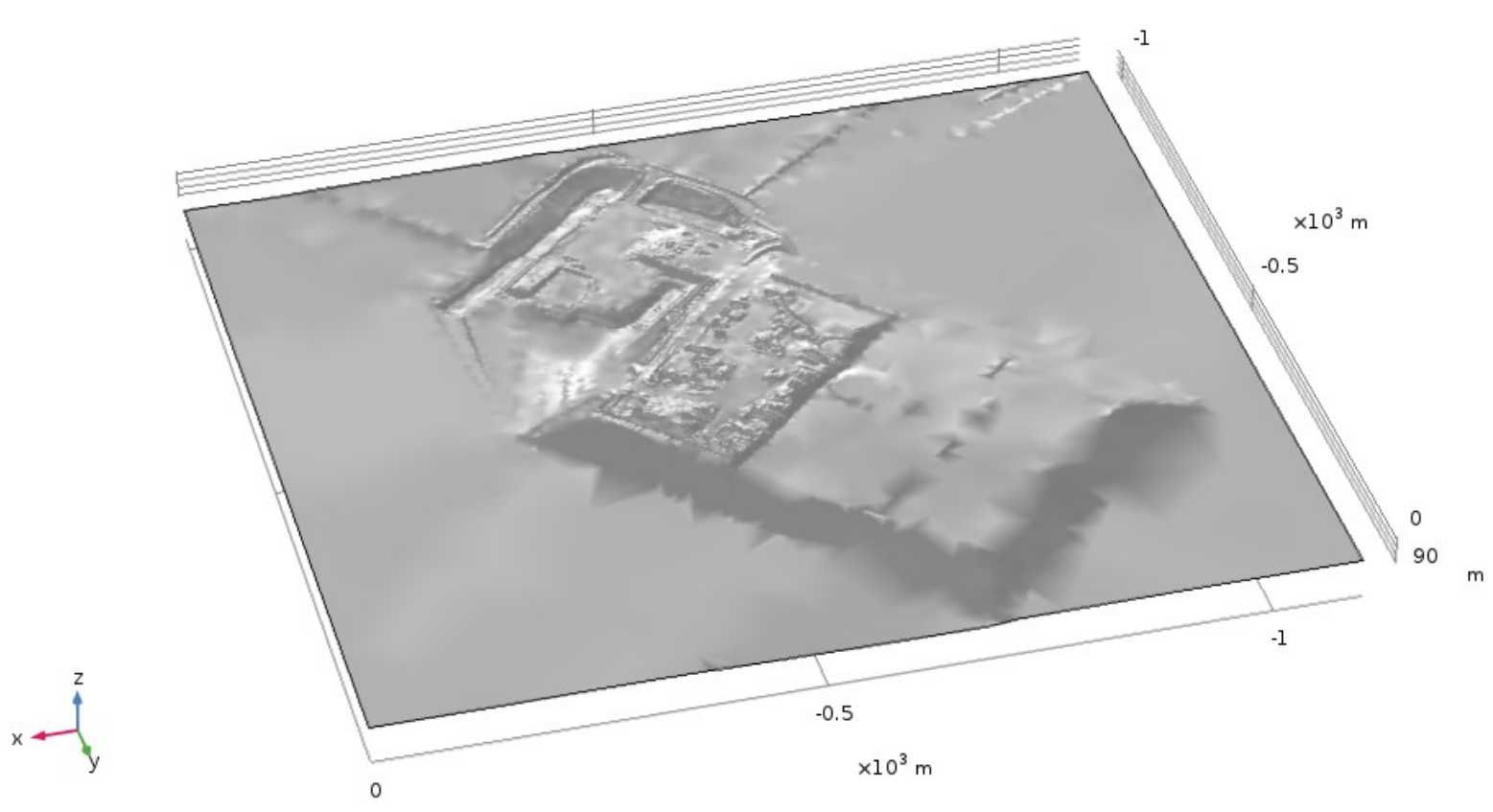

Figure 57: 3D Meshing of Ste. Sophie GIS Data in COMSOL Multiphysics 